\title{
WILEY-VCH
}

\section{Graphene-based hybrid functional materials}

Cosimo Anichini, Paolo Samori*

Dr. C. Anichini, Prof. P. Samorì

Université de Strasbourg, CNRS, ISIS, 8 alleé Gaspard Monge, 67000 Strasbourg, France.

E-mail: samori@unistra.fr;

Keywords: graphene, hybrid materials, graphene oxide, graphene hybrids, functional materials, functionalization

Graphene is a 2-dimensional material combining numerous outstanding physical properties, including high flexibility and strength, extremely high thermal conductivity and electron mobility, transparency, etc., which make it a unique testbed to explore fundamental physical phenomena. Such physical properties can be further tuned by combining graphene with other nanomaterials or (macro)molecules to form hybrid functional materials, which by design can display not only the properties of the individual components but exhibit new properties and enhanced characteristics arising from the synergic interaction of the components. The implementation of the hybrid approach to graphene also allows to boost the performances in a multitude of technological applications. In this review, we report the hybrids formed by graphene combined with other low-dimensional nanomaterials of diverse dimensionality $(0 \mathrm{D}$, 1D and 2D) and (macro)molecules, with emphasis on the synthetic methods. The most important applications of these hybrids in the fields of sensing, water purification, energy storage, biomedical, (photo)catalysis and opto(electronics) are also reviewed, with a special focus on the superior performances of these hybrids compared to the individual, not-hybridized components.

\section{Introduction}

Graphene is a monatomic-thick layer of hexagonally arranged carbon atoms with $\mathrm{sp}^{2}$ hybridization. This 2-dimensional (2D) material can be seen as an individual layer of graphite: 


\section{WILEY-VCH}

in fact, it is known since the first X-ray diffraction studies that graphite is formed by many graphene layers closely stacked together via van der Waals interactions. ${ }^{[1]}$ Graphene is also the basic building-block of two other carbon allotropes: theoretically, a graphene layer can be rolled to form hollow closed cylinders called carbon nanotubes (CNTs) and wrapped into hollow spheres called fullerenes. Graphene was isolated in 2004 by Andre Geim and Konstantin Novoselov who developed a simple method to place atomically thick layers of graphene onto thermally oxidized silicon wafers $\left(\mathrm{Si} / \mathrm{SiO}_{2}\right)$ by mechanical cleavage of graphite with common adhesive tape. ${ }^{[2]}$ Their seminal studies on the material revealed a range of outstanding physical and chemical properties. Such ground-breaking work was awarded with the Nobel Prize in physics in 2010. Significantly, graphene has rapidly become the most investigated and employed material in science, ${ }^{[3]}$ being subject of more than $120000^{*}$ papers by 2020 . These unique properties include huge specific surface area $\left(2630 \mathrm{~m}^{2} \mathrm{~g}^{-1}\right)^{[4]}$, excellent electron mobility (up to $2 \times 10^{5} \mathrm{~cm}^{2} \mathrm{~V}^{-1} \mathrm{~s}^{-1}$ at room temperature $)^{[5]}$, extremely high thermal conductivity $(5000 \mathrm{~W}$ $\left.\mathrm{m}^{-1} \mathrm{~K}^{-1}\right)^{[6]}$, remarkable mechanical strength and flexibility (Young's modulus $\left.\sim 1 \mathrm{TPa}\right)^{[7]}$, high transparency ( $2.3 \%$ of light absorption in the visible and infrared) ${ }^{[8]}$ and notable stability. The range of possible applications for such a unique material is vast; however, the use of pristine graphene produced by the aforementioned mechanical cleavage of graphite has been limited to the academic environment due to the very low throughput of the method. Other synthetic techniques to produce large and high-quality graphene layers via "bottom-up" approach, such as chemical vapour deposition (CVD) and epitaxial growth on $\mathrm{SiC}$, have been developed, yet, in these cases, the so-produced graphene is expensive and thus struggle to enter in end-products of the market. On the other hand, graphene can be obtained also in form of small "flakes"

\footnotetext{
"Source: Web of Science (Titles containing: "graphene")
} 


\section{WILEY-VCH}

dispersed in a liquid media by the so-called liquid-phase exfoliation (LPE) methods. ${ }^{[9]}$ These methods share a common characteristic which is the exfoliation of graphite in a solvent which is capable of overcoming the van der Waals forces that keep the layers together. To help the solvent intercalation among graphite layers to overcome the strong interactions between the adjacent layers, eventually leading to the complete separation of the graphene layers, an external force must be applied. The choice of the external assisting force and the exfoliation parameters used allows adjusting the characteristics of the exfoliated according to a trade-off between quality, resulting sizes and yield of monolayers flakes. A method related to LPE is the electrochemical exfoliation of graphite, in which graphene is exfoliated by applying an electrical potential to an electrochemical cell in which graphite is used as anode or cathode in presence of an electrolyte-containing medium. Compared to other LPE methods, the electrochemical exfoliation usually produces larger graphene flakes in higher yield. ${ }^{[10]}$ Finally, on the extreme the trade-off between quality, dimensions and yield of monolayers there is a material that can be produced at relatively low cost and in large volumes, yet substantially differs from graphene, so much so that it was given a different name: graphene oxide (GO). Graphene oxide can be obtained from the exfoliation in water under moderate sonication of graphite oxide, which is a strongly oxidized form of graphite. Graphene oxide has a structure similar to graphene, yet contains numerous $\mathrm{sp}^{3}$ carbons and oxygens in form of epoxy, hydroxyl, carbonyl and carboxyl groups. These functionalities and the presence of $\mathrm{sp}^{3}$ carbons make GO hydrophilic, electrically insulating (conductivity below $1 \times 10^{-4} \mathrm{~S} \mathrm{~m}^{-1}$ ) ${ }^{[1]}$ and reactive, while graphene is hydrophobic, conductive and inert. By reducing GO thermally, chemically or electrochemically most of the $\mathrm{sp}^{3}$ carbons can be re-converted back to $\mathrm{sp}^{2} \mathrm{C}$ and most of the oxygen containing groups can be eliminated. The material is usally referred to as reduced graphene oxide ( $\mathrm{rGO}$ ), and has characteristics half-way between $\mathrm{GO}$ and pristine 


\section{WILEY-VCH}

graphene, with much higher electrical conductivity (up to $\left.10^{5} \mathrm{~S} \mathrm{~m}^{-1}\right)^{[12]}$, lower reactivity and hydrophilicity compared to GO.

Because of its properties, unfunctionalized graphene mainly finds applications in photonic and electronics $^{[13]}$. Nonetheless, soon after the graphene discovery, while many of its properties were still unknown, some scientists started wondering what could happen if one combines this wonder material with other materials or molecules with different properties to form a hybrid. The implementation of the hybrid approach to graphene not only allowed improving the performances in the two aforementioned applications, but also paved the way to its use in a broader variety of applications, including sensing, (photo)catalysis and electrocatalysis, water purification, supercapacitors, batteries, drug-delivery, bioimaging, etc. Hybrid materials are obtained by coupling of two or more materials sharing similar dimensions on a small, nanometric scale, where electronic interactions are predominant. The close proximity of the components in the hybrid, which are intimately connected and interact together through noncovalent or covalent interactions, and their similar dimensions produce a large interfacial area. ${ }^{[13]}$ This interface is what differentiate the hybrids from composites, and, by enabling interfacial processes such as energy and electron transfers, is the responsible of the synergistic effect which makes the hybrid present improved performances compared to those of the individual components and, often, even to the emergence of new properties.

Conversely, composites of graphene are those materials composed by a polymeric or ceramic matrix and graphene as filler. In this material the content of graphene is rather low (usually below $5 \mathrm{wt} \%$ ) and the filler is usually blended in the matrix by mechanical mixing. ${ }^{[14]}$ The role of graphene in these composites is to confer electrical and thermal conductivity to the composite and/or increase their mechanical characteristics. Some of these composites have already found several application in the market, however the performances of most of these products are not 


\section{WILEY-VCH}

largely superior to those of composites with carbon nanotubes or carbon fibres. ${ }^{[15]}$ The main reason to these performances is that often the graphene used has low quality, with high thickness and small lateral sizes, being more similar to graphite than to graphene. ${ }^{[15 b]}$

This review describes the most enlightening approaches to create hybrid materials based on graphene with tailored properties and their primary fields of application. Graphene based hybrids are firstly classified in view of the type of exploited second component including nanomaterials (whose 0D, 1D, 2D dimensionality is exploited as categorizing criteria) and (macro)molecules, with an accent on both covalent and non-covalent functionalization. Subsequently, some relevant applications of these hybrids materials in the fields of sensing, water purification, (photo)catalysis, biomedical, energy storage and optoelectronics will be highlighted. For each application we will show how these hybrids can represent a potential solution to most of the challenges of the field.

\section{Classes of hybrid assemblies based on graphene}

\subsection{Assemblies of graphene with other nanomaterials}

Nanomaterials are materials which have at least one of their physical dimensions below 100 $\mathrm{nm}$. The great interest towards nanomaterials comes from the fact that usually, nanomaterials possess properties (e.g. optical, electrical, chemical...) that are highly different from their bulk counterpart. Graphene has been combined with other nanomaterials with the aim of creating a new hybrid material possessing new properties. Graphene can be bonded to the other nanostructures via covalent or non-covalent interactions. However, the non-covalent interactions usually prevail, since they are simpler to achieve not needing the previous chemical functionalization of the nanomaterial and, eventually, graphene. ${ }^{[16]}$ Two main synthetic strategies exist: the ex-situ and the in-situ techniques. In the ex-situ technique graphene and the 


\section{WILEY-VCH}

second nanomaterial are firstly synthesized separately with the desired morphology and dimensions, and then they are coupled together, by mixing the two materials in liquid-phase or by deposition of one of the two materials onto/into the other. Often, the surface of the nanomaterial and/or graphene must be modified to favour the supramolecular interaction or the chemical bonding between the two components, assuring a good coupling between the two materials. The advantage of the ex-situ method is that, by synthesizing the two materials separately, it is possible to achieve a fine control over their morphology, structure, and dimensions. ${ }^{[13]}$ Conversely, in the in situ technique the nanomaterial is grown directly onto the surface of graphene. In the case of inorganic nanomaterials, such as nanoparticles or nanowires, the graphene is generally mixed in solution with a precursor of the second nanomaterial to be grown (usually a metal salt or a metalorganic compound) and possibly a templating agent and then the precursor is reduced yielding the generation of the inorganic nanomaterial. The reduction may be chemical, thermal, electrochemical or induced by light. ${ }^{[13]}$ The specific adsorption of the nanomaterial precursor on the surface of graphene allows the nanomaterial to grow selectively onto graphene. The interaction of the precursor with graphene is usually driven by electrostatic and Van der Waals forces, in particular in GO the oxygen atoms favour the nucleation of the nanomaterials on its surface.

In the following section, some examples of hybrid assemblies of graphene and other nanomaterials, classified in view of their dimensionality (i.e. 0D, 1D, and 2D) are presented.

\subsubsection{Assemblies of graphene and OD nanostructures}

Hybrid composites made by combining graphene with functional OD nanomaterials such as nanoparticles (NPs), quantum dots (QDs), and nanocrystals are probably among the most explored because of their facile production and increased functionalities and properties that the OD materials confer to the assembly. Nanoparticles are materials that are confined to the 


\section{WILEY-VCH}

nanoscale in all three dimensions. They can be widely found in nature and are the first nanomaterials known and employed. In fact, they were already used in the prehistory. ${ }^{[17]}$ Quantum dots are semiconducting nanoparticles. Nanoparticles and QDs can be prepared by bottom-up or top-down approaches, although the bottom-up growth from chemical precursors is more widespread. ${ }^{[18]}$ The properties of nanoparticles depends not only on their chemical composition, but also on their size, shape, number of facets, crystallinity, etc. ${ }^{[17]}$ For instance, depending on their size and ligand present on their surface, Au NPs may exhibit a range of different optical, catalytic and electrical properties. ${ }^{[19]}$

As aforementioned, the hybrid graphene-nanoparticles can be assembled by in-situ or ex-situ methods. Typically, the assembly of the two materials in the ex-situ method or the growth of the $0 \mathrm{D}$ nanomaterial onto the graphene surface in the in-situ method takes place in liquid media. Then the assembly may be deposited onto a substrate by different techniques. For instance, Hayakawa et al. ${ }^{[20]}$ prepared a hybrid of $\mathrm{rGO}$ and $\mathrm{Cu}_{2} \mathrm{ZnSnS}_{4}$ by both ex-situ and in-situ methods. In the first case, the previously synthesized $\mathrm{Cu}_{2} \mathrm{ZnSnS}_{4} \mathrm{NPs}$ were mixed with $\mathrm{rGO}$ in cyclohexane, and the formation of the hybrid was favoured by the addition of oleylamine as surfactant and by ultrasonicating the dispersion. In the second case the $\mathrm{Cu}_{2} \mathrm{ZnSnS}_{4}$ NPs were directly grown on rGO by a one-pot solvothermal method using oleylamine, sulphur and the metal salt precursors. The characterization of the two materials showed that in both cases similar NPs for dimensions and crystallinity were obtained, but in the hybrid synthesized by insitu method, the NPs coverage of rGO was higher (Figure 1 a). The reducing agent necessary to reduce the metal salt to form the metal NPs can also be used to simultaneously reduce GO to rGO in the in-situ synthesis. For example, Chandra et al. ${ }^{[21]}$ synthesized a water-dispersible hybrid $\mathrm{rGO} /$ magnetite $\left(\mathrm{Fe}_{3} \mathrm{O}_{4}\right)$ NPs. The assembly was synthesized in situ by the simultaneous reduction of $\mathrm{GO}, \mathrm{FeCl}_{2}$ and $\mathrm{FeCl}_{3}$ that led to the formation of $10 \mathrm{~nm}$ magnetite nanoparticles 


\section{WILEY-VCH}

on the rGO sheets (Figure 1 b). GO and the iron salts were dispersed in water, then $\mathrm{NH}_{3}$ was added to precipitate the $\mathrm{Fe}^{2+} / \mathrm{Fe}^{3+}$ ions onto the GO surface and finally hydrazine was added to both reduce GO and the Fe ions to $\mathrm{rGO}$ and magnetite NPs. Alternatively, the 0D nanostructures can be deposited or grown on graphene already laying on a substrate, for example, by employing a Layer-by-Layer (LbL) approach. ${ }^{[22]}$ Mao et al. ${ }^{[23]}$ prepared in this fashion a hybrid of rGO decorated with $\mathrm{SnO}_{2}$ nanocrystals which was used to sense $\mathrm{NO}_{2}$ and $\mathrm{NH}_{3}$ gases. In this case, rGO was firstly deposited by drop-casting onto interdigitated gold electrodes on $\mathrm{Si} / \mathrm{SiO}_{2}$ substrate and then the $\mathrm{SnO}_{2}$ nanocrystals previously prepared in a mini-arch reactor were deposited on the rGO drop-cast film by electrostatic force directed assembly. The Layer-byLayer deposition technique allows assembling multilayer structures of two or more materials. The deposition is often driven by electrostatic interactions. For instance, Xiao et al. ${ }^{[22]}$ prepared a multi-layer structure of modified rGO flakes and CdS QDs by LbL deposition that was used for photoelectrochemical and photocatalytic applications. To assemble the multi-layer structure a fluorine doped tin oxide (FTO) conductive substrate was alternatively soaked in a solution of the negatively charged CdS QDs and in the suspension of the positively charged modified rGO flakes (Figure 1 c-d). 


\section{WILEY-VCH}
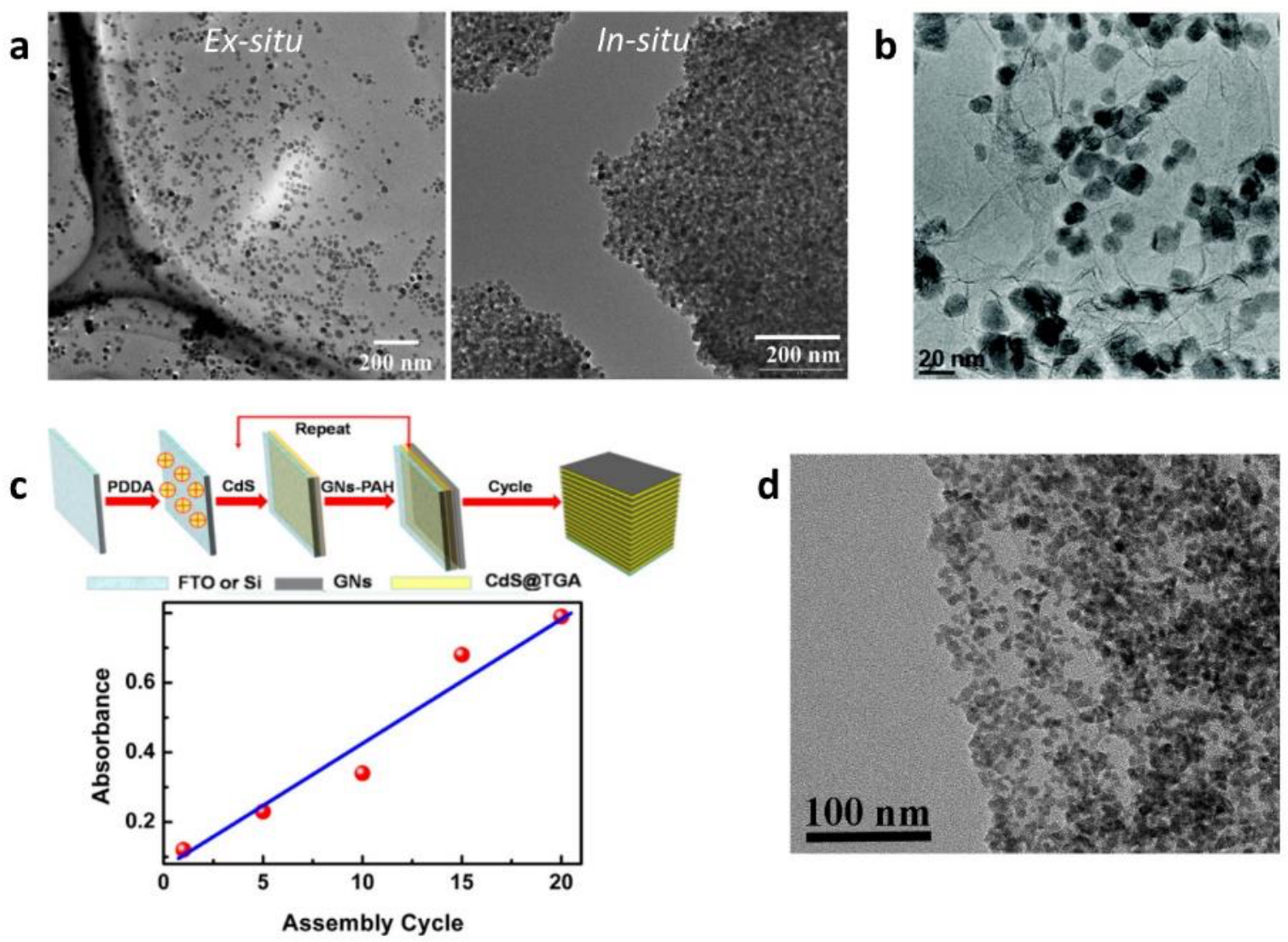

Figure 1: (a) Transmission electron microscopy (TEM) images of $\mathrm{rGO} / \mathrm{Cu}_{2} \mathrm{ZnSnS}_{4}$ NPs hybrid produced by an ex-situ (left) and in-situ (right) methods. Adapted from Ref. ${ }^{[20]}$ (b) TEM image of $\mathrm{rGO} / \mathrm{Fe}_{3} \mathrm{O}_{4} \mathrm{NPs}$ hybrid synthesized via an in-situ one-pot method. Adapted from Ref. ${ }^{[21]}$ (c) Schematic of the LbL synthesis of a multi-layer modified rGO (GNs-PAH)/CdS QDs hybrid (top) and absorbance of the hybrid multi-layer film on FTO as a function of the number of LbL cycles (bottom). (d) TEM image of the multi-layer GNs-PAH/CdS QDs hybrid after 5 cycles of deposition. Adapted from Ref. ${ }^{[22]}$

While in the ex-situ method the advantage is an easier control over the characteristics of the nanoparticles, the in-situ synthesis can produce a change in the size of the NPs because of the effect of graphene on the nucleation and growth of the nanoparticles. ${ }^{[13]}$ In particular, a reduction of the dimensions of the NPs is often observed because of the so-called heat sink effect. ${ }^{[2]}$ The extremely high thermal conductivity of graphene facilitate the dissipation of the heat produced during the nanoparticles crystallization, preventing this heat to be used to further grow the NPs. For instance, this phenomenon has allowed growing Pt NPs with size below 0.5 


\section{WILEY-VCH}

nm on graphene nanosheets (Figure 2 a). ${ }^{[24 b]}$ The extremely small size of these Pt NPs strongly enhanced the electrocatalytic activity of the hybrid towards the oxidation of methanol.

The catalytic activity of noble metal nanoparticles (Ag, Au, Pd and Pt NPs) has been exploited to assemble hybrids with graphene used in both sensing and catalysis. [24b, 25] For instance, hybrids of graphene with Pd and Pt NPs have been extensively explored to sense $\mathrm{H}_{2}$ gas by virtue of the well-known catalytic activity of these two metals towards the adsorption of hydrogen. ${ }^{[25 a-c]}$ Hybrid materials composed of graphene and semiconductive metal oxide nanoparticles, or quantum dots $\left(\mathrm{ZnO}, \mathrm{SnO}_{2}, \mathrm{In}_{2} \mathrm{O}_{3}, \mathrm{Cu}_{2} \mathrm{O}\right.$, etc. $)$ have also proved to enhance the sensing performances of graphene to $\mathrm{NO}_{2}$, ethanol, $\mathrm{H}_{2} \mathrm{~S}$ and other gases. ${ }^{[23,26]}$ For example, Zhou et al. ${ }^{[26 \mathrm{a}]}$ developed a $\mathrm{GO} / \mathrm{Cu}_{2} \mathrm{O}$ NPs hybrid for the sensing of $\mathrm{H}_{2} \mathrm{~S}$. The $\mathrm{Cu}_{2} \mathrm{O}$ NPs were hydrothermally grown in situ on the GO surface by exploiting the electrostatic interaction between the negatively charged groups on GO surface and the positively charged $\mathrm{Cu}^{2+}$ ions. The suspension of the hybrid material was then drop-cast onto a $\mathrm{Si} / \mathrm{SiO}_{2}$ substrate exposing prepatterned gold electrodes. The $\mathrm{GO} / \mathrm{Cu}_{2} \mathrm{O}$ hybrid exhibited better performances compared to the isolated components, with a high $11 \%$ sensitivity to $5 \mathrm{ppb}$ of $\mathrm{H}_{2} \mathrm{~S}$ and high selectivity (Figure $2 \mathrm{~b}-\mathrm{c})$. The better performances were due to an increased active surface for the adsorption of $\mathrm{H}_{2} \mathrm{~S}$ and an increase in the charge transfer efficiency. Hybrids of graphene with fluorescent QDs, such as CdS and CdTe have also been used to prepare highly sensitive fluorescent sensors for metal ions and (bio) molecules and as platforms for bioimaging and photodynamic therapy. ${ }^{[27]}$ Hybrids of graphene and semiconductive QDs and $\mathrm{TiO}_{2}$ have been used also in photocatalysis and electrocatalysis. For example, Lv et al. fabricated a noble metal free hybrid of graphene decorated with $\mathrm{CdS}$ and $\mathrm{TiO}_{2} \mathrm{NPs}$ for the photocatalytic hydrogen evolution from methanol. ${ }^{[28]}$ The material was prepared via one-pot solution method and exhibited enhanced photocatalytic activity compared to the bare nanoparticles (with a hydrogen production rate 4 


\section{WILEY-VCH}

times higher) and to Pt co-catalyst. Magnetic nanoparticles ( $\mathrm{Fe}, \mathrm{Fe}_{3} \mathrm{O}_{4}, \mathrm{Mn}_{3} \mathrm{O}_{4}$, etc.) have been often combined with graphene (GO and rGO in particular) with the purpose of adsorbing pollutants from water. ${ }^{[21,27 a, 29]}$ In this case the nanoparticles have a dual role. On the one hand, they produce an increase of the surface area and of the active binding sites in the hybrid that enhance the adsorption capabilities. On the other hand, after that the hybrid is dispersed in the polluted water and has adsorbed the pollutant the magnetic nanoparticles consent the hybrid to be easily separated and removed from water by using a magnet (Figure $\mathbf{2} \mathrm{d}$ ). GO and rGO have also been combined with magnetic nanoparticles for drug delivery: in this case the magnetic nanoparticles can be exploited to guide the GO hybrid platform drug-carrier to the target tissue in the body. ${ }^{[30]}$ Furthermore, they allow the location of the carrier to be visualized with magnetic resonance imaging and can be used as well as therapeutic agent by photothermal therapy. ${ }^{[31]}$ Other applications of magnetic nanoparticles are in the field of electrocatalysis and photocatalysis. ${ }^{[16]}$ For example, $\mathrm{Fe}_{3} \mathrm{C}$ nanoparticles encapsulated into few-layers graphene sheets, produced by one-step autoclave pyrolytic synthesis, have been used for the electrocatalytic oxygen reduction reaction. ${ }^{[32]}$ In this case the graphene encapsulation protects the oxidation sensitive $\mathrm{Fe}_{3} \mathrm{C}$ from the external environment.

Among the nanoparticles, core-shell nanoparticles, which are composed by an inner core and an outer layer of different materials, are particularly interesting because they exhibit both the properties of the two constituting materials. ${ }^{[33]}$ For instance, Zhu et al. ${ }^{[34]}$ employed graphene decorated with core@double-shell magnetic nanoparticles ( $\left.\mathrm{Fe} @ \mathrm{Fe}_{2} \mathrm{O}_{3} @ \mathrm{Si}-\mathrm{S}-\mathrm{O}\right)$, for the removal of $\mathrm{Cr}(\mathrm{VI})$ from water. The hybrid material was synthesized in situ starting from graphene sheets, sodium dodecylbenzenesulfonate as surfactant and $\mathrm{Fe}(\mathrm{CO})_{5}$. The reactants were dispersed in DMF and heated at reflux. Then the hybrid material was thermally annealed at $500{ }^{\circ} \mathrm{C}$ for $2 \mathrm{~h}$ in $\mathrm{H}_{2} / \mathrm{Ar}$ atmosphere to form the final core-shell structure of the NPs (Figure 


\section{WILEY-VCH}

2 e-f). The magnetic inner cores of the NPs allowed the hybrid to be easily separated from water with a magnetic field while the external Si-S-O shell exhibited a strong binding affinity to $\mathrm{Cr}(\mathrm{VI})$, which was adsorbed from water with high efficiency. In another example a hybrid of core-shell Ag@Co and Ag@Ni NPs supported on rGO has been used for hydrogen generation from the hydrolysis of ammonia borane. ${ }^{[25 e]}$ The material was synthesized via an in situ onestep procedure, using methylamine borane for the simultaneous reduction of GO and the coreshell metallic NPs and showed improved catalytic performances compared to the same NPs supported on different materials.

a

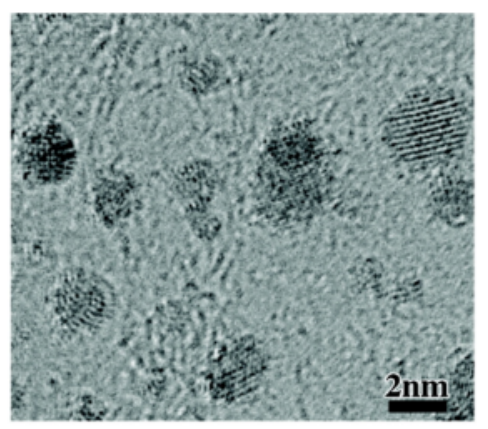

b
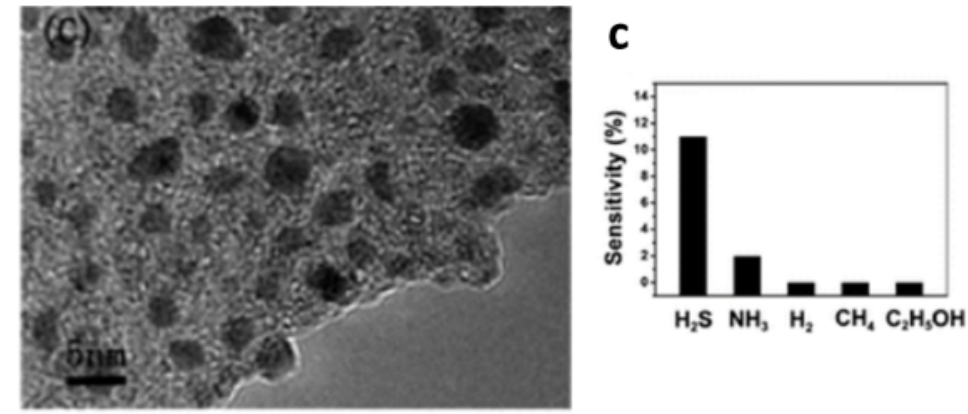

d

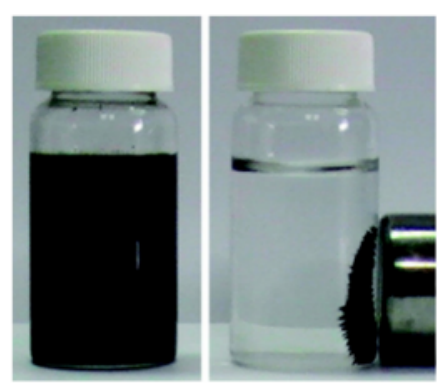

e

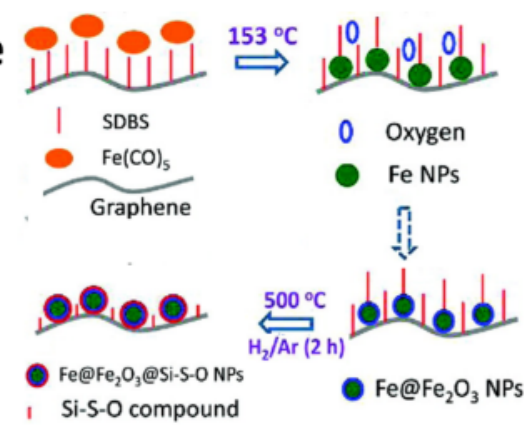

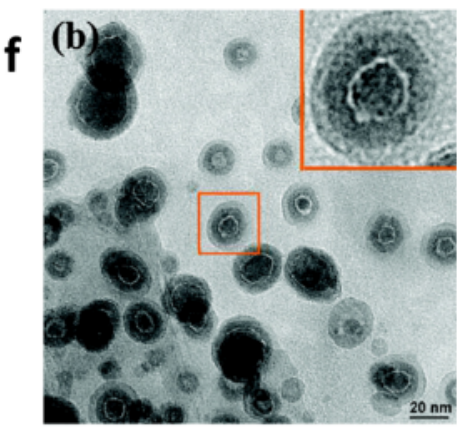

Figure 2: (a) TEM image of a graphene nanosheets/Pt NPs hybrid, showing the small $(<1 \mathrm{~nm})$ dimension of the NPs. Adapted from Ref. ${ }^{[24 b]}$ (b) TEM image of $\mathrm{GO} / \mathrm{Cu}_{2} \mathrm{O}$ hybrid $\mathrm{H}_{2} \mathrm{~S}$ sensor and (c) Sensitivity of the hybrid towards different vapours and gases. Adapted from Ref. [26a] (d) Photos showing the dispersed magnetic $\mathrm{rGO} / \mathrm{Fe}_{3} \mathrm{O}_{4} \mathrm{NPs}$ hybrid (left) and the hybrid separated from water by using a permanent magnet. Adapted from Ref. ${ }^{[21]}$ (e) Schematic of the synthesis and (f) TEM image of a graphene/Fe@ $\mathrm{Fe}_{2} \mathrm{O}_{3} @ \mathrm{Si}-\mathrm{S}-\mathrm{O}$ NPs hybrid. Adapted from Ref. [34]

\subsubsection{Assemblies of graphene and $1 D$ nanostructures}




\section{WILEY-VCH}

One dimensional (1D) nanomaterials are those materials characterized by at least two dimensions in the nanoscale (below $100 \mathrm{~nm}$ ) yielding therefore to a large aspect ratio. Most representative components of this class of nanostructures include nanotubes (NTs), nanowires (NWs), and nanorods. These materials can exhibit conducting, semiconducting, or insulating properties. Carbon nanotubes are one of the allotropes of carbon; discovered before graphene in 1993, they are hollow cylinders made of carbon atoms with $\mathrm{sp}^{2}$ hybridization forming a honeycomb lattice. The walls of the cylinders may be composed by either one single atomic thick layer of $\mathrm{C}$ atoms or by two or more layers. Conversely, nanowires are thin wires with diameter usually below $100 \mathrm{~nm}$ and can be made of several materials. Hybrid materials based on graphene assembled with 1D nanomaterials have been extensively studied in the last 10 years as testified by the continuous increase of the number of works related to these materials which can be found in the literature. In particular, hybrid materials based on graphene and conductive 1D materials such as metallic nanowires and carbon nanotubes (CNTs) have been extensively investigated as transparent conductive electrodes for optoelectronics ${ }^{[35]}$ and supercapacitors. ${ }^{[36]}$ Assemblies of graphene with semiconducting nanowires have found many applications as materials for batteries ${ }^{[37]}$ and sensing devices. ${ }^{[35 a, 38]}$ The interaction between CNTs and graphene is often driven by the strong $\pi$ - $\pi$ stacking that is formed between these two aromatic carbon allotropes, ${ }^{[13,16]}$ however, in some cases these have also been bonded covalently. ${ }^{[36 a]}$ These hybrids can be assembled in-situ, usually by growing the CNTs onto the surface of graphene, ${ }^{[39]}$ or more often the two materials are synthesized separately and then combined ex-situ. [36b, 38a, 40] Similarly, hybrids composed of metallic nanowires or semiconducting inorganic nanowires and graphene have been synthesized both in-situ, by growing the nanowires onto graphene ${ }^{[41]}$ or by growing the graphene via CVD onto or on top of the nanowires ${ }^{[37 \mathrm{c}, 42]}$, and ex-situ. ${ }^{[37 \mathrm{~b}, 38 \mathrm{~b}, 43]}$ 


\section{WILEY-VCH}

Assemblies of graphene and carbon nanotubes or metal nanowires ( $\mathrm{Cu}$ NWs and $\mathrm{Ag} \mathrm{NWs}$ ) have been the subject of intense research endeavour to generate transparent and opaque conductive electrodes. The network of metal NWs or CNTs is electrically conductive and their discontinuous morphology enables light transmission through the voids between NWs/CNTs. On the other hand, graphene is also conductive and possesses high transmittance in the visible light, thus contributing to the conductivity of the electrode, while maintaining good transparency. However, in most of the cases graphene holds an additional function: its inertness and low reactivity, together with its layered structure makes it almost impermeable to most molecules. ${ }^{[44]}$ This implies that, if the graphene layer is deposited on top of the easily oxidizable metal nanowires, it can help to protect the nanowires from oxidation.

Hybrids of graphene with 1D materials, and in particular CNTs, may benefit from the increased surface area and conductivity, making them particularly useful for supercapacitors, whose performances rely on a large specific surface. Supercapacitors are among the first and foremost important applications of graphene. ${ }^{[45]}$ The electrodes constituting a supercapacitor should store charges at the liquid-electrode interface, thus conductive materials exhibiting a large surface area are required for such application. One of the first reports of a supercapacitor based on a hybrid of graphene and CNTs was published in 2009 by Yu and Dai. ${ }^{[36 b]}$ They produced a multilayer self-assembled $\mathrm{GO} / \mathrm{CNTs}$ hybrid by $\mathrm{LbL}$ deposition of positive rGOpoly(ethyleneimine) (PEI) and negatively charged acid-oxidized multiwalled carbon nanotubes. The hybrid showed a specific capacitance of $120 \mathrm{~F} \mathrm{~g}^{-1}$. Later Jung et al. fabricated another supercapacitor based on a composite of stacked graphene and CNTs in which the graphene and CNTs were covalently tethered. ${ }^{[36 \mathrm{a}]}$ The two components were bonded together by amide bond obtained by reacting a NHS ( $N$-hydroxysuccinimide) functionalized GO with amine-functionalized CNTs. Subsequently, the aqueous solution of GO-CNTs hybrid was 


\section{WILEY-VCH}

reduced to rGO-CNT by a microwave hydrothermal (MWHT) method in presence of hydrazine and ammonia and then filtered to obtain the stacked structure, which exhibited a very high volumetric capacitance of $165 \mathrm{~F} \mathrm{~cm}^{-3}$. Li et al fabricated via an extended filtration assisted method a flexible asymmetric supercapacitor based on a 3D architecture in which the negative electrode was composed of stacked graphene sheets spaced by CNTs. ${ }^{[36 \mathrm{~d}]} \mathrm{CNTs}$ had a dual role, acting as a hard spacer to prevent restacking of graphene sheets but also as a conductive and robust network to facilitate the electrons collection and transport in order to fulfil the demand of high-rate performance of the asymmetric supercapacitor. Graphene hybrids with 1D materials have also been employed as electrodes for lithium batteries. For instance, Wang et al. fabricated a hierarchical architecture employed as anode of a Li-ion battery in which Si NWs encapsulated with CVD graphene were sandwiched between rGO sheets. ${ }^{[37 \mathrm{c}]}$ The Si NWs were firstly synthesized via a CVD vapour-liquid-solid (VLS) method and then CVD graphene was growth on their surface (Si NWs@G), finally the Si NWs@G and GO were filtered together and thermally reduced to obtain the stacked Si NWs@G@rGO structure (Figure 3 a-c). In the structure, the CVD graphene coating prevented the direct exposure of encapsulated silicon to the electrolyte and enabled the structural and interfacial stabilization of silicon nanowires, while the conductive rGO sheets accommodated the volume change of embedded Si NW@G and maintained the structural and electrical integrity of the assembly. Li et al. fabricated a freestanding electrode for Lithium sulphur batteries based on a 3D assembly of rGO and titanium nitride nanowires (TiN NWs). ${ }^{[37 \mathrm{a}]}$ The electrode was assembled by firstly dispersing $\mathrm{H}_{2} \mathrm{Ti}_{3} \mathrm{O}_{7}$ nanowires and $\mathrm{GO}$ in water, to which sodium ascorbate was added to reduce GO and form a hydrogel. Then the $3 \mathrm{D}$ gel was annealed at $800{ }^{\circ} \mathrm{C}$ in $\mathrm{NH}_{3}$ atmosphere to obtain the composite rGO/TiN NWs. In 2018 Xue et al. reported the assembly of a 3D hierarchical structure based on rGO and Ag NWs employed as anode for Li-metal batteries. ${ }^{[37 b]}$ The porous 


\section{WILEY-VCH}

3D structure was formed by freeze-drying an ice-templated hydrogel of GO and Ag NWs and then reducing with hydrazine vapours the resulting aerogel (Figure $3 \mathrm{~d}-\mathrm{e}$ ).

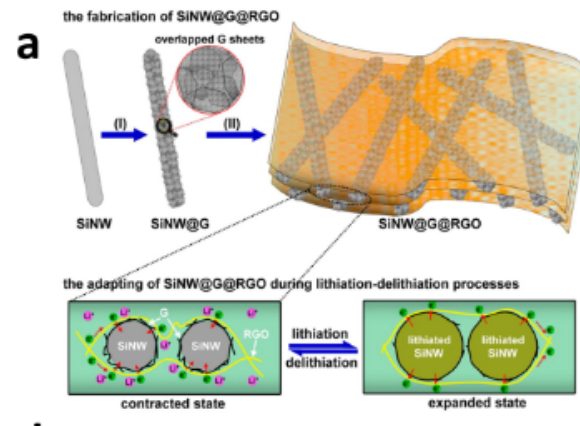

d

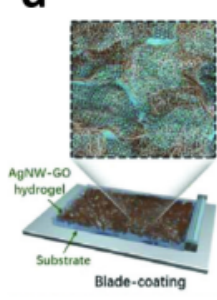

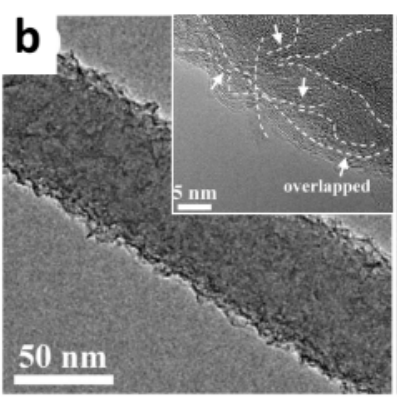
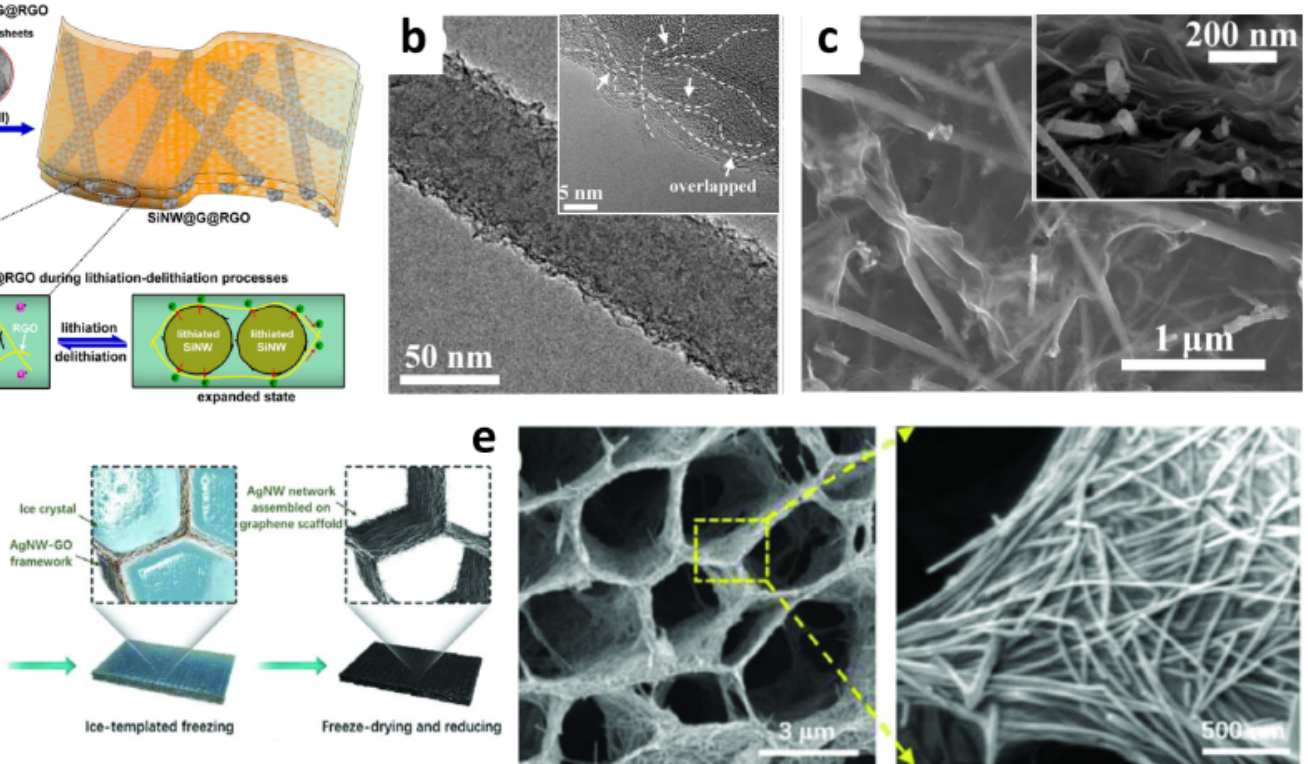

Figure 3 : (a) Schematic of the fabrication (upper panel) and adapting (lower panel) of $\mathrm{Si}$ NWs@CVD graphene/rGO hybrid. (b) TEM images of the Si NWs@CVD graphene and (c) SEM images of the hybrid Si NWs@CVD graphene/rGO. Adapted from Ref. [37c](d) Schematic of the fabrication process of the rGO-AgNWs hybrid aerogel and (e) SEM images at different magnifications of the aerogel. Adopted from Ref. ${ }^{[37 b]}$

Assemblies of graphene and 1D materials have been also largely employed in sensing devices, by taking advantage of the increased surface area, which is essential for sensing, and the additional functionality that the 1D material may confer to the hybrid. In 2010 Jeong et al. produced a flexible $\mathrm{NO}_{2}$ sensor using a CNTs/rGO hybrid film. ${ }^{[39 a]} \mathrm{A}$ rGO film was deposited onto a plastic substrate with pre-patterned electrodes, on which vertically aligned CNTs were deposited on top by CVD. Yi et al. ${ }^{[42 \mathrm{a}]}$ assembled an ethanol sensor by hydrothermally growing semiconductive vertically aligned $\mathrm{ZnO}$ nanorods on a metal bottom electrode and depositing CVD few-layer graphene on top as the top contact. A hybrid based on vertically aligned $\mathrm{ZnO}$ nanorods, hydrothermally grown on a rGO film was employed by Cuong et al. to sense $\mathrm{H}_{2} \mathrm{~S} .{ }^{[41]}$ Hybrid assemblies have also been used to sense (bio)molecules in liquid media. Woo et al. 


\section{WILEY-VCH}

prepared a hybrid $\mathrm{rGO} / \mathrm{CNT}$ electrochemical sensor for the determination of $\mathrm{H}_{2} \mathrm{O}_{2} \cdot{ }^{[38 \mathrm{a}]}$ The material was prepared by the simultaneous reduction of GO dispersed in water with hydrazine in presence of dispersed CNTs. Then the hybrid was deposited on a glassy carbon electrode. The hybrid displayed a large electrochemical surface area and fast electron transfer properties which allowed determining of $\mathrm{H}_{2} \mathrm{O}_{2}$ in concentrations as low as $9.4 \times 10^{-6} \mathrm{M}$. In another example, He et al. fabricated a dopamine electrochemical sensor based on a hybrid $\mathrm{rGO} / \mathrm{MnO}_{2}$ NWs electrode. ${ }^{[38 b]}$ The hybrid was prepared by mixing GO and $\mathrm{MnO}_{2} \mathrm{NWs}$ in solution and then drop-casting the self-assembled composite onto a glassy carbon electrode. The hybrid was then electrochemically reduced to $\mathrm{rGO} / \mathrm{MnO}_{2} \mathrm{NWs}$ and used to sense dopamine, with a detection limit of $1 \mathrm{nM}$ and good selectivity even in the presence of high concentrations of ascorbic acid and uric acid.

\subsubsection{Assemblies of graphene and other $2 D$ materials}

Despite the outstanding electronic properties of graphene, the difficulty in producing highquality graphene in large quantities at low cost and the lack of a bandgap in its electronic structure have prevented the efficient use of graphene for several electronic and optoelectronic applications. To overcome this limitation and to diversify the physical properties, in recent years the research activity has been broadened to other 2D layered materials, and in particular to transition metal dichalcogenides (TMDs), hexagonal boron nitride (h-BN) and phosphorene. Hexagonal boron nitride (h-BN) is a remarkable layered insulator with a structure similar to that of graphene. TMDs are compounds of the type $\mathrm{MX}_{2}$, with $\mathrm{M}$ a transition metal atom (Mo, W, etc.) and $\mathrm{X}$ a chalcogen atom ( $\mathrm{S}, \mathrm{Se}$, or Te), which can be made atomically thick. Phosphorene is a single or few-layer thick version of the thermodynamically stable black phosphorus. Both TMDs and phosphorene are semiconductors with bandgaps between 1 and 2 


\section{WILEY-VCH}

$\mathrm{eV}^{[46]}$ Interestingly, hybrid structures can be made by combining graphene with one or more of these $2 \mathrm{D}$ materials, by stacking them together in a vertical alignment or by stitching them in a lateral alignment (Figure 4); these hybrids structures are often referred as 2D heterostructures or van der Waals heterostructures, since the different components are held together by van der Waals forces which are the same forces that keep together the different layers in the bulk material. ${ }^{[46-47]}$ These heterostructures allow not only overcoming the inherent limitations of every single component, but also the combination of multiple $2 \mathrm{D}$ materials may lead to the emergence of novel peculiar properties which are extrinsic to the single components.

\section{Graphene 2D Heterostructures}
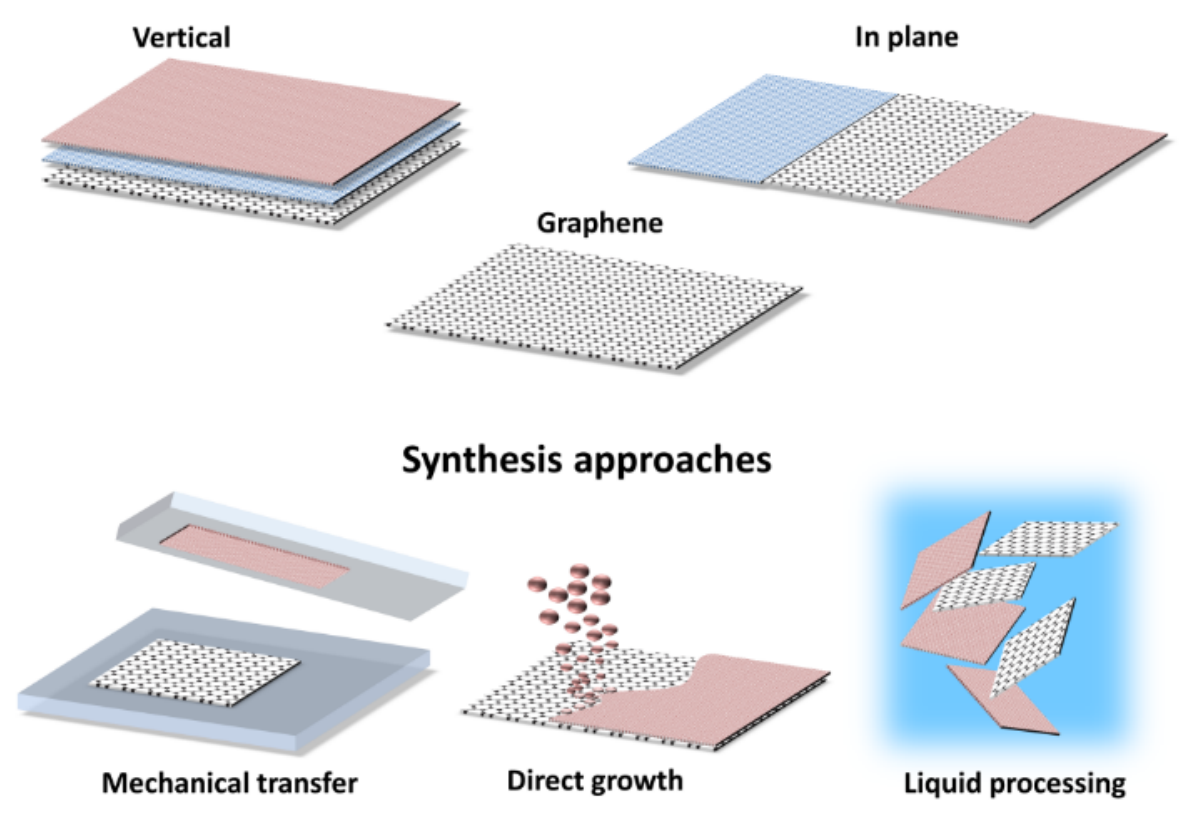

Figure 4: Schematic overview of the different kinds of graphene 2D heterostructures introduced in this paragraph, and methods usually employed to fabricate them.

The first example of a graphene-based heterostructure was reported in 2010 by Dean and coworkers. ${ }^{[48]}$ The group fabricated a transistor on a vertical heterostructure in which exfoliated single and bi-layer graphene were placed on top of thin hBN crystal by using a mechanical transfer process. The two layers were closely attached together by van der Waals forces. The use of a $2 \mathrm{D}$ layer of $\mathrm{h}-\mathrm{BN}$ for supporting the graphene flake brought new interesting 


\section{WILEY-VCH}

characteristics: for example, the graphene displayed carrier mobilities almost an order of magnitude greater than devices on $\mathrm{SiO}_{2}$ and presented reduced roughness, different reactivity and intrinsic doping. Since the publication of this work, several hundreds of papers and dozens of reviews have been published about lateral and vertical heterostructures based on h-BN and graphene which can be produced by mechanical transfer methods, assembling in liquid phase of the liquid exfoliated graphene and h-BN and direct growth by vapour deposition methods. ${ }^{[46,}$ ${ }^{47 b, 49]}$ One interesting characteristic of h-BN is its atomically smooth surface that is almost completely free of dangling bonds and charge traps, which is the reason of the superior carrier mobility that Dean et al. had observed for graphene flakes stacked onto h-BN. In plane hBN/graphene heterostructures may present semiconducting behaviour and tunable band-gap, antiferromagnetism and low thermal conductance. ${ }^{[49 b]}$ On the contrary, vertical stacks of graphene and h-BN exhibited unique physical properties. For example, since graphene and h$\mathrm{BN}$ share an almost-but not perfectly identical crystal lattice, when they are superimposed they generate a periodic moiré pattern which is at the origin of some quantic effect experientially observed in these sample for the first time, such as the Hofstadter's butterfly effect ${ }^{[50]}$ and the superlattice Dirac cone (Figure 5 b-c). ${ }^{[51]}$ Furthermore, when the angle between the lattice of h-BN and graphene is small a bandgap $(<20 \mathrm{meV})$ appears in the last. ${ }^{[52]}$ The graphene can also be fully encapsulated between two h-BN layers, in order to protect it from the environment. H-BN has been reported also as ultrathin dielectric gate, for field-effect transistor based on vertical h-BN/graphene heterostructures. ${ }^{[53]}$ 


\section{WILEY-VCH}

a

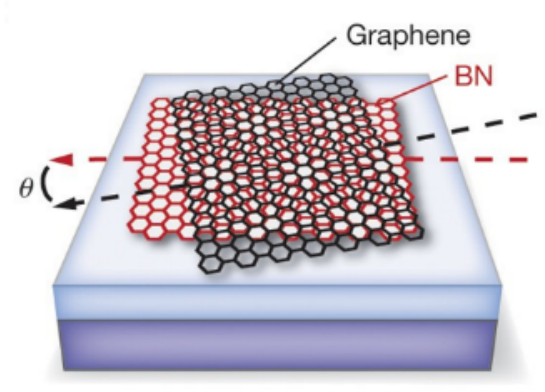

b

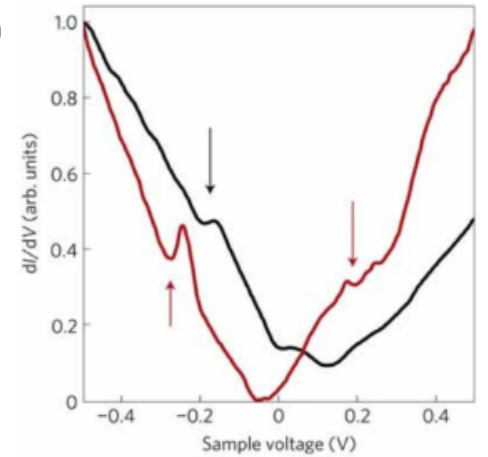

C

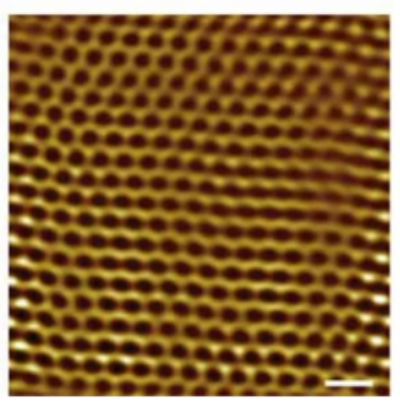

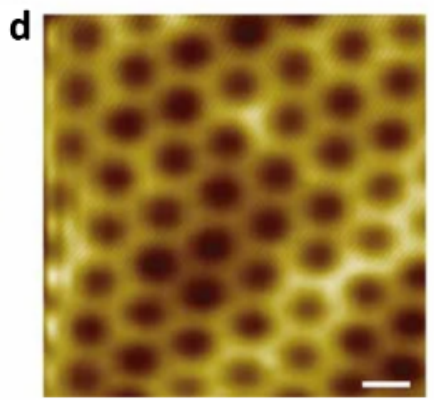

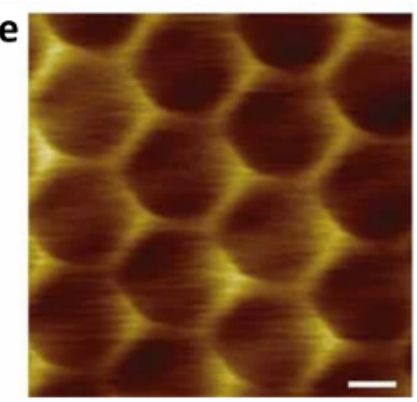

Figure 5 : (a) Sketch of graphene on h-BN showing the emergence of a moiré pattern. The moiré wavelength varies with the mismatch angle, $\theta$. Adapted from Ref. ${ }^{[50]}$ (b) Experimental $\mathrm{dI} / \mathrm{dV}$ curves of the vertical heterostructure $\mathrm{h}-\mathrm{BN} / \mathrm{Graphene}$ for two different moiré wavelengths, $9.0 \mathrm{~nm}$ (black) and $13.4 \mathrm{~nm}$ (red). The dips in the $\mathrm{dI} / \mathrm{dV}$ curves are marked by arrows. (c-e) STM topography images showing $2.4 \mathrm{~nm}$ (c), $6.0 \mathrm{~nm}$ (d) and $11.5 \mathrm{~nm}$ (e) moiré patterns. The scale bar is $5 \mathrm{~nm}$. Adapted from Ref. ${ }^{[51]}$

Vertical heterostructures can be fabricated by using different techniques that include mechanical transfer methods, chemical growth, and liquid phase assembly. As aforementioned, mechanical "wet" transfer was firstly demonstrated by Dean et al. for the production of graphene transistor devices supported by h-BN. ${ }^{[48]}$ The first step was the mechanical exfoliation of a thin flake of h-BN on a substrate, along with the exfoliation of graphene on a poly(methyl methacrylate) (PMMA) thin membrane supported on a $\mathrm{Si} / \mathrm{SiO}_{2}$ substrate. The second step was the transfer of the PMMA membrane with the exfoliated graphene flake, on top of a transparent glass slide. Finally, under an optical microscope, the glass slide was placed hanging on the top of the h-BN, with the graphene flake facing down. With the aid of a micromanipulator, the glass slide was moved to perfectly align the graphene flake over the h-BN, then both were brought 


\section{WILEY-VCH}

into contact and the two flakes were attached closely by van der Waals forces (Figure 6a). This method has been extensively used also to produce vertical heterostructures of mechanically exfoliated graphene with TMDs and phosphorene. ${ }^{[47 \mathrm{a}]}$ Furthermore, by repeating this procedure it is possible to stack multiple layers of different materials, with increasing complexity and functionality (Figure 6b).${ }^{[46,54]}$ However, since the many steps involved in the transfer method, contaminants are often present at the interface between the two $2 \mathrm{D}$ materials. These contaminants may be removed after annealing, though not all the materials can tolerate annealing without degrading. ${ }^{[46]}$ For this reason, dry transfer techniques have been developed in which the contact of the interfaces with solvents or polymers is avoided (Figure 6d). ${ }^{[55]}$ Liquid phase exfoliation of graphene and other 2D materials can be used to obtain vertical randomly stacked heterostructures. ${ }^{[56]}$ Typically the bulk materials are exfoliated in liquid phase and, after centrifugation steps, stable suspension of single and few layers 2D materials are obtained, then the suspensions are mixed together in different ratios. The electrostatic and van der Waals interaction between the graphene flakes and the other 2D materials flakes drive their self-assembly forming stacked heterostructures, which are finally separated from the liquid.

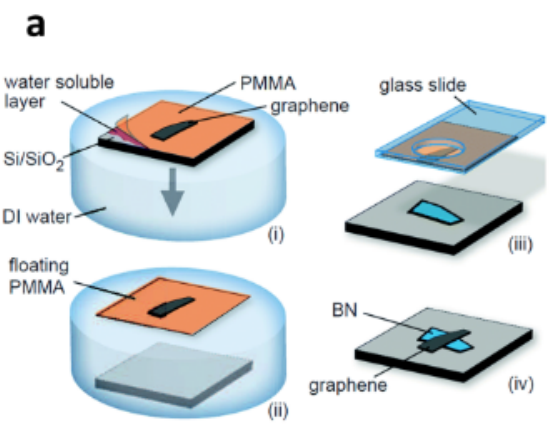

b

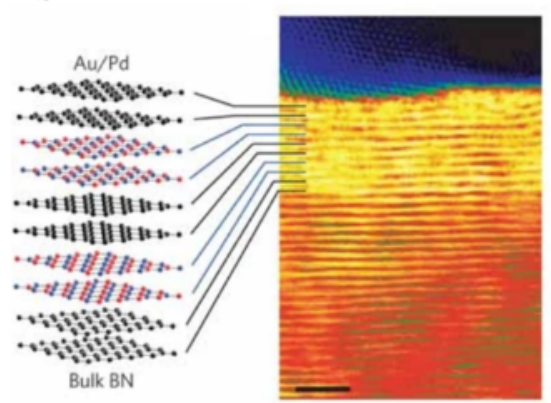

C

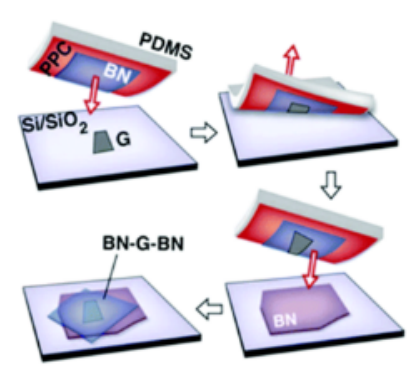

Figure 6 : (a) Schematic illustration of the "wet" transfer process used to fabricate grapheneon-BN devices. Adapted from Ref. ${ }^{[48]}$ (b) Sectional STEM image and schematic of a heterostructure fabricated by the sequential stacking of graphene and h-BN layers. Adapted from Ref. ${ }^{70}$ (c) Schematics for the polymer-free stacking technique of $h-B N / G / h-B N$ aided by van der Waals interactions. Adapted from Ref. [55a] 


\section{WILEY-VCH}

Chemical synthesis is another method used to produce vertical 2D heterostructures. Mechanical transfer methods produce high-quality heterostructures, suitable for fundamental research purposes. However, the small size of the obtained heterostructures, the laborious procedures and low output volume hinder their applications outside the research area. On the other hand, liquid-phase methods are easily scalable and produce high-volume of materials. However, heterostructures fabricated by these methods are usually randomly stacked and thus not suitable for certain purposes. Chemical vapour deposition is a technique to grow high-quality pristine 2D materials in large areas and can be employed to fabricate large-area vertical heterostructures. Individual layers of 2D materials can be grown separately and then stacked together with various transfer methods to produce large heterostructures that found application in optoelectronics. ${ }^{[47 \mathrm{a}]}$ Also in this case, the transfer methods, although simpler than the transfer of mechanically exfoliated flakes that necessitate sub-micrometric precision and electron-beam lithography technique, involve the use of solvents and polymers which brings contamination of the surfaces. Furthermore, the transfer of large-area CVD films often results in cracks and wrinkles. ${ }^{[47 \mathrm{a}]}$ It is possible to overcome this problem by directly growing one kind of $2 \mathrm{D}$ material onto another 2D material used as substrate. The first example of direct growth was reported by Liu et al. and consisted in the CVD growth of h-BN on CVD graphene. ${ }^{[57]}$ As aforementioned, graphene and h-BN are very similar, they are isoelectronic and have a very similar crystal lattice; furthermore, both can be easily grown by CVD on copper. First, graphene was grown on $\mathrm{Cu}$ foil at $950{ }^{\circ} \mathrm{C}$ using hexane as a precursor. Then h-BN was grown on the graphene through the decomposition of ammonia borane at $1000{ }^{\circ} \mathrm{C}$. However, Raman spectroscopy revealed the damage of the graphene during the second CVD growth. The growth of graphene on h-BN has been more widely studied than the opposite, since h-BN represents an excellent dielectric substrate for graphene, making these structures more useful for electronic 


\section{WILEY-VCH}

applications, and h-BN resists better to the second CVD growth compared to graphene. In this framework various methods have been developed; in an early example, CVD graphene has been grown by CVD using benzene as a precursor at $800{ }^{\circ} \mathrm{C}$ onto an epitaxial h-BN thin film on Ni. ${ }^{[58]}$ Yan et al. developed a smart method to deposit bilayer graphene films directly onto insulating substrates including h-BN. ${ }^{[59]}$ The h-BN was coated with a thin polymeric film, followed by the evaporation of $500 \mathrm{~nm}$ of Nickel on top of the polymer. Then the substrate was annealed in reducing atmosphere of $\mathrm{H}_{2} / \mathrm{Ar}$ at $1000{ }^{\circ} \mathrm{C}$ for a few minutes. The polymeric film was converted into graphene, which can be exposed after the etching of Ni. One of the main challenges regarding the growth of graphene onto $\mathrm{h}-\mathrm{BN}$ it is the modest catalytic activity of the latter, which renders necessary the presence of some exposed metal. ${ }^{[49 b]}$ However, recently the number of methods to grow graphene layers onto h-BN, with and without metal catalysts, has increased; these methods rely on techniques such plasma enhanced CVD (PE-CVD) and low pressure CVD (LPCVD) and make use of different carbon feedstocks, included liquids. ${ }^{[46-47,}$ ${ }^{49 \mathrm{~d}]}$ It is worth noting the work of Song et al. who recently developed a procedure for the growth of patterned graphene on 2D h-BN. ${ }^{[60]}$ The group firstly deposited PMMA seeds in copper, then by using LPCVD and ammonia borane as precursor they grew the h-BN layer and finally, using benzoic acid as precursor, they grew graphene whose domains nucleated exactly over the underlying PMMA seeds.

In addition to h-BN vertical heterostructures of graphene and TMDs have also been extensively studied. Although they possess quite different properties, vertical stacks of graphene/TMD/graphene have found different applications, such as in FET with high $\mathrm{I}_{\mathrm{on}} / \mathrm{I}_{\text {off }}$ ratios, in optoelectronics and photovoltaics. In addition to the numerous transfer methods that have been reviewed previously, these heterostructures have been synthesized via various direct growth procedures. For instance, Ling et al. showed that $\mathrm{MoS}_{2}$ monolayers could be grown 


\section{WILEY-VCH}

onto graphene from sources of $\mathrm{MoO}_{3}$ and $\mathrm{S}$, using fluorinated phthalocyanine as nucleation seeds. ${ }^{[61]}$ Other common graphene/TMD heterostructures have been prepared also by van der Waals epitaxy (VDWE) method, which is the growth of layered materials onto clean and smooth surfaces with no dangling bond, even in presence of a large lattice mismatch between the two layers. The result is a heterostructure with a high-quality interface due to the absence of surface defects and dangling bonds, in which the two layers are held together only by van der Waals forces. Vertical heterostructures of graphene and TMDs have found many applications as ultrafast and ultrasensitive photodetectors ${ }^{[62]}$ and light harvesting, ${ }^{[63]}$ memory devices, ${ }^{[64]}$ light-emitting diodes ${ }^{[65]}$ and highly efficient FET. ${ }^{[46-47]}$ In most of the cases graphene act as source, drain and gate electrodes, and as electron collector, while the TMD layers as the proper semiconductor. Flexible and transparent devices based on vertical heterostructures supported on PET have also been produced. ${ }^{[46]}$ On the contrary, stacking of graphene (including GO and rGO) and other 2D materials (such as TMDs, phosphorene and hBN) obtained by liquid-phase processing have found interesting applications in the field of energy storage, with applications in supercapacitors, lithium-ion batteries and sodium-ion batteries. ${ }^{[46,66]}$ For example, Bissett et al. fabricated a graphene/MoS 2 hybrid supercapacitor from liquid phase exfoliated material. ${ }^{[67]}$ Dispersions of graphene and $\mathrm{MoS}_{2}$ were mixed together and filtered to create thin flexible electrodes in a symmetrical supercapacitor based on aqueous electrolyte. The performances of the supercapacitor based on the graphene/ $\mathrm{MoS}_{2}$ composite with ratio 1:3 were greatly enhanced over either individual component. This was attributed to a combination of effects, on the one hand, $\mathrm{MoS}_{2}$ flakes could intercalate and prevent restacking of the larger graphene sheets. On the other hand, $\mathrm{MoS}_{2}$ enhanced conductivity and pseudocapacitance of the hybrid by increasing the ion adsorption sites. For lithium-ion batteries anodes, aerogels papers based on rGO and $\mathrm{MoS}_{2}$ have also been 


\section{WILEY-VCH}

employed ${ }^{[68]}$ First hydrogel of $\mathrm{rGO}$ and $\mathrm{MoS}_{2}$ were formed with hydrothermal methods that involve the in situ simultaneous reduction of GO and formation of the hybrid structure, then the samples were freeze-dried to form the aerogel. In these examples, $\mathrm{MoS}_{2}$ mostly acted as a spacer facilitating the Li-ion intercalation in the electrode. Composites of phosphorene and graphene have been successfully employed in sodium-ion batteries; in fact, phosphorene can react with sodium and lithium to form $\mathrm{Na}_{3} \mathrm{P}$ and $\mathrm{Li}_{3} \mathrm{P}$, increasing ion storing potential of the electrode and thus the specific capacity of the battery that can reach the $2596 \mathrm{mAh} \mathrm{g}^{-1} \cdot{ }^{[56 \mathrm{~b}]} \mathrm{In}$ these composite with phosphorene graphene plays a role as an elastic buffer, accommodating the big changes in volume of the electrode that deforms reversibly without breaking.

Lateral, in-plane heterostructures in which unidimensional junctions are created between the different materials have also attracted much interest. ${ }^{[46-47,49 b]}$ The combination of more $2 \mathrm{D}$ materials on the same plane allows achieving ultra-thin devices. However, the development of these structures has been slower than for vertical heterostructures for two main reasons. The first reason is the challenging connection of the two materials on the same plane, since thin unidimensional interfaces need to be perfectly in contact. This implies the lack of a straightforward method, such as the mechanical stacking for vertical heterostructures, to produce lateral heterostructures. To ensure the intimate connection between the $2 \mathrm{D}$ materials, all the methods rely on the bottom-up synthesis of at least one of the materials. The second reason is that these interfaces can only be realized by in situ techniques, in which one of the materials starts to grow directly from the edge of the other. Since the conditions for the direct growth of the second material from the edge of the first material may damage the latter, compatible techniques had to be developed. Nevertheless, in the last years, the advances in the techniques allowed the growth of these in-plane heterostructures that possess interesting properties. The first lateral heterostructures realized, and also the most studied, are based on h- 


\section{WILEY-VCH}

$\mathrm{BN}$ and graphene because the two materials share many characteristics, and these are the structures with the most interesting properties. As aforementioned, graphene and h-BN have the same structure and a lattice mismatch of just $1.7 \%$, furthermore, they both grow well on $\mathrm{Cu}$ and Ni substrates. The first structures realized was a random heterostructure formed by h$\mathrm{BN}$ and graphene domains growth simultaneously by $\mathrm{CVD}$ from $\mathrm{CH}_{4}$ and ammonia borane. ${ }^{[69]}$ This method suffered from a poor control over the size and shape of the domains of each material, as well as over the quality of the interfaces. More recently, numerous methods which allow controlling the size, shape and position of each material have been developed. In general, these are all two-steps growth processes and involve the use of some kind of mask and/or lithographic process to pattern the first material before the deposition of the second (Figure 7). ${ }^{[46-47,49 c, 70]}$ It is worth to note that in some conditions the second material grows with an epitaxial orientation respect to the first material, and it has been shown that the two materials are strongly connected by covalent bonds. ${ }^{[49 c]}$ Few examples of in-plane heterostructures of graphene and TMDs also exist in the literature; however, the number of studies is still small compared to the structures with h-BN. ${ }^{[71]}$ In general, all these structures have been fabricated by CVD growth of the first material, followed by patterning and by the CVD growth of the second material. Differently from the case of $h-B N$, graphene and TMDs have different structure and crystal lattice. These differences lead to the overlapping of the two materials at the interfaces, where the two materials interact by van der Waals forces. Perfect unidimensional interfaces between the two layers are more challenging and have not been realized yet. Lateral heterostructures of graphene and TMDs have been used to fabricate atomically thin FETs that possess good Ohmic contact between the TMD and the two source and drain electrodes made of monolayer graphene. ${ }^{[71 \mathrm{~b}, 71 \mathrm{c}]}$ 


\section{WILEY-VCH}

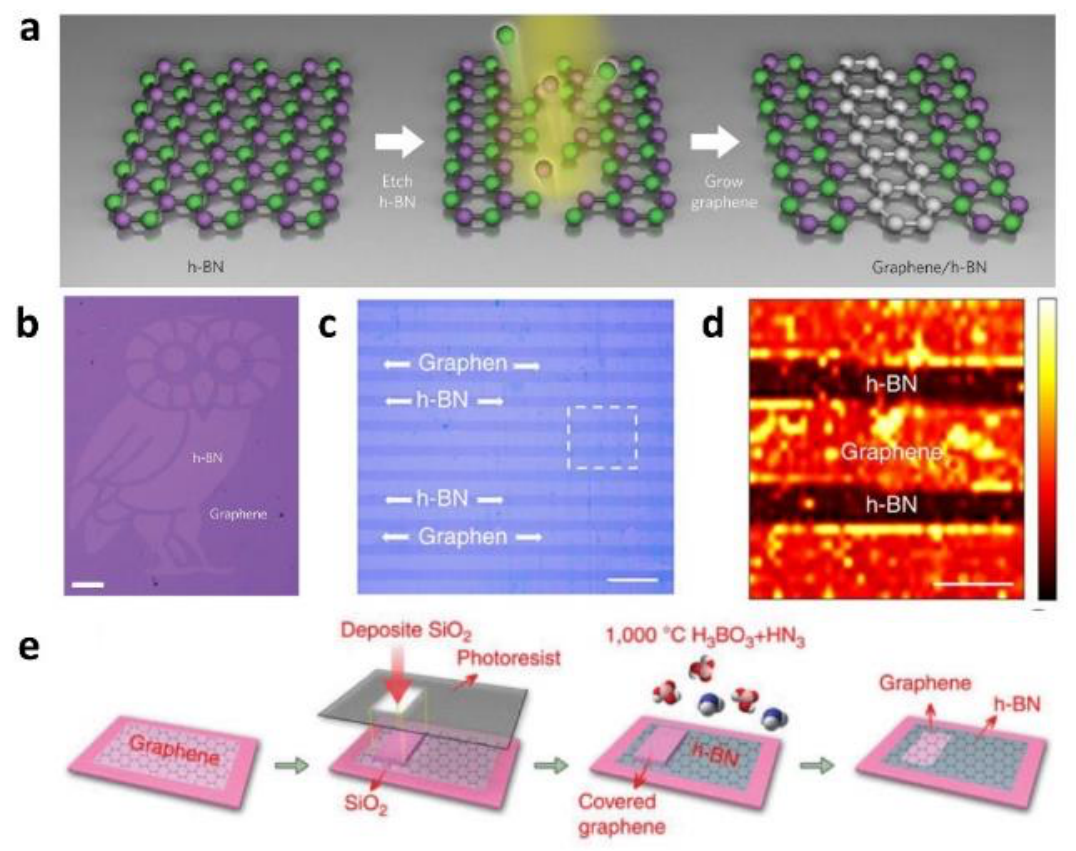

Figure 7: (a) Schematic of the fabrication process of a patterned in-plane graphene/h-BN heterostructure. (b) Optical microscope image of the so obtained heterostructure on a $\mathrm{Si} / \mathrm{SiO}_{2}$ substrate. Adapted from Ref. ${ }^{[70 a]}$ (c) Optical microscope image of graphene/h-BN alternative strips on $\mathrm{Si} / \mathrm{SiO}_{2}$ substrate . (d) Raman mapping of the marked area in (c) at $2 \mathrm{D}$ peak $\left(2700 \mathrm{~cm}^{-1}\right)$ of graphene. (e) Schematic of the fabrication of the graphene/h-BN patterned inplane heterostructure involving the CVD growth of graphene, a photolithographic process to pattern the graphene and conversion of the unprotected graphene to h-BN. Adapted from Ref. [70b]

\subsection{Graphene modified with (macro)molecules}

The chemical modification of graphene opens the possibility to tailor its properties, such as the electrical properties, to add new functionalities and to enhance its processability, solubility, and compatibility with other materials. For example, graphene can become a semiconductor with an adjustable bandgap via the functionalization of its basal plane. ${ }^{[72]}$ The functionalization of graphene can be carried out at the covalent or not covalent level. A difference should be made between pristine graphene, graphene oxide and reduced graphene oxide, because the presence of some oxygen-containing groups in the last two increases the number of available reactions and kind of non-covalent interactions with other molecules in comparison with pristine graphene. In fact, a limited number of methods have been reported for the covalent 


\section{WILEY-VCH}

functionalization of pristine graphene; these methods usually involve very reactive species generated in situ such as nitrenes and free radicals. ${ }^{[73]}$ If the purpose is to very mildly affect the electronic properties of pristine graphene and just increase its processability and solubility the non-covalent functionalization should be preferred. Molecules can physisorb on pristine graphene exploiting van der Waals and $\pi-\pi$ interactions, among which $\pi-\pi$ stacking interactions have a higher magnitude. On the other hand, GO, and to a lesser extent rGO, possess residual oxygen containing groups in form of epoxides, hydroxyls, carboxylic acids and ketones on their surfaces that make available a wide series of reactions. Furthermore, because of the oxygen groups and carboxyls, in GO also the type of non-covalent interactions with molecules which can be used are more numerous and include ionic, electrostatic interactions and hydrogen bonds.

\subsubsection{Chemical covalent functionalization of graphene}

The functionalization of pristine graphene sheets with organic molecules has been developed for several purposes. In the first instance, the effort could be justified by the need to improve the dispersibility of pristine graphene in common organic solvents. The dispersion of graphene in organic solvents or water can be indeed seen as the first and crucial step towards the formation of graphene-based hybrid materials. The covalent nature of the bond endows to the hybrid a notable robustness. Furthermore, chemical modification with functional molecules is a viable approach to confer to graphene new properties, such as photoluminescence, semiconductivity or selective interactions with certain analytes. In most cases, when molecules are covalently attached on the graphene surface, a modification of the aromatic structure of graphene takes place, thereby perturbing its electrical properties. Among the various possible perturbations, the possibility of opening a tunable bandgap is the most explored for its implications in the fields of electronics and photonics. Pristine graphene is a material composed 


\section{WILEY-VCH}

exclusively of carbon atoms with $\mathrm{sp}^{2}$ hybridization, likewise its structurally related allotropes graphite, carbon nanotubes and fullerenes. For this reason, graphene has benefited from the numerous reactions previously developed for fullerenes and carbon nanotubes. However, the curved structure and misaligned $\pi$-orbitals of fullerenes and carbon nanotubes make them more reactive than graphene which as a result of its fully delocalized $\pi$ system is rather inert.

In the case of pristine graphene, free radicals or dienophiles are used to covalently attach the $\mathrm{C}=\mathrm{C}$ bonds of its basal plane. The use of diazonium salts has been one of the first methods to functionalize pristine graphene. These salts usually are freshly prepared before the reaction, because of their instability. The functionalization reaction takes place through the decomposition of diazonium cation that releases $\mathrm{N}_{2}$ and the formation of an aryl radical that attacks an $\mathrm{sp}^{2}$ carbon of graphene. ${ }^{[7]}$ Tour et al. made use of this reaction to decorate graphene nanoribbons with nitrophenyls (Figure 8 a). ${ }^{[75]}$ The groups showed that during chemical functionalization the conductivity of graphene decreased due to the re-hybridization of carbon atoms from $\mathrm{sp}^{2}$ to $\mathrm{sp}^{3}$ and consequent disruption of the aromatic system. Similarly, Niyogi and co-workers covalently attached nitrophenyls to graphene via diazonium chemistry. ${ }^{[76]}$ The reaction created a controllable band gap in graphene, opening its application as a semiconducting nanomaterial. Benzoyl peroxide is another route to generate free aryl radicals reactive with graphene. Liu et al. employed benzoyl peroxide to functionalize mechanically exfoliated graphene. ${ }^{[77]}$ The reaction was conducted in toluene solution and initiated photochemically by focussing a laser onto the graphene surface and the success was monitored by following the appearance of the D band in the Raman spectrum of graphene (Figure 8 b-d) 


\section{WILEY-VCH}
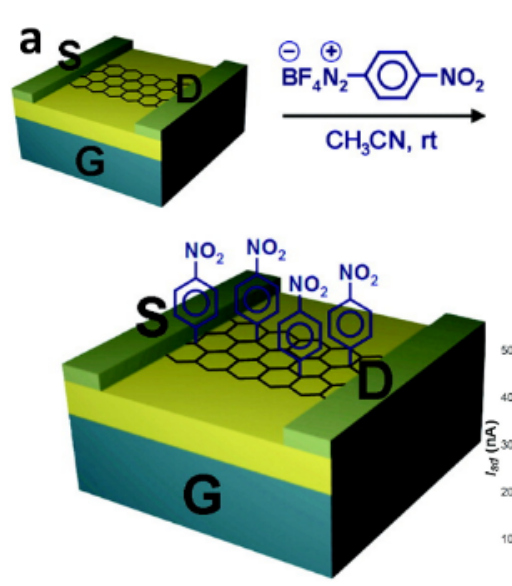

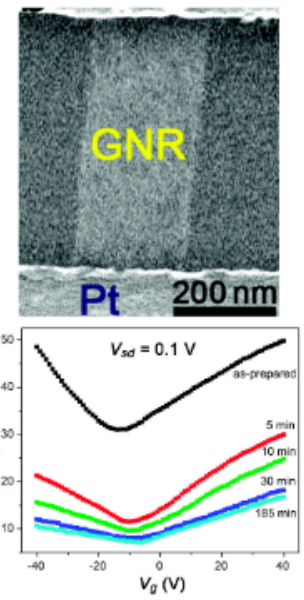

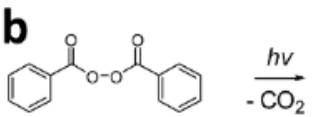

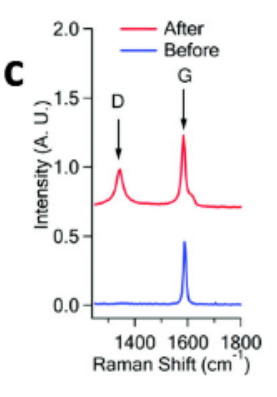

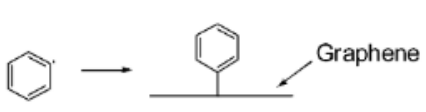

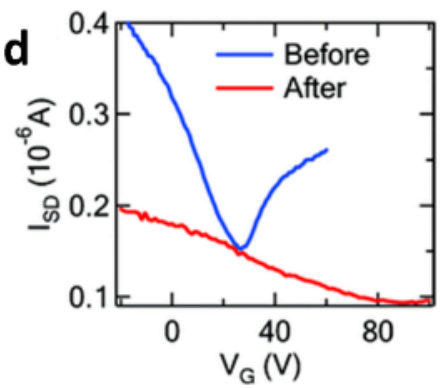

Figure 8: (a) Schematic representation of the covalent functionalization of a graphene nanoribbon (GNR) with nitrophenyls. Insets: SEM image of the GNR device and transfer curves of the device recorded at $\mathrm{V}_{\text {sd }}=0.1 \mathrm{~V}$ for the same electronic device in panel (a) after several consecutive grafting experiments. Adapted from Ref. ${ }^{[75]}$ (b) Schematic reaction of graphene with benzoyl peroxide photochemically activated. (c) Raman spectra and (d) transfer curves of graphene before and after the functionalization. Adapted from Ref. ${ }^{[77]}$

Cycloadditions are the second class of possible reactions with pristine graphene. Azomethine ylides are one of the most common dienophiles that have been successfully applied in the functionalization of graphene through 1,3 dipolar cycloaddition (Figure 9 a). ${ }^{[78]}$ This reaction has been extensively used by Prato and his group to functionalize CNTs with a variety of derivatives that conferred applications in the fields of biotechnology, drug delivery and optoelectronic devices. ${ }^{[74]}$ The azomethine ylide can be formed in situ by condensation of an aldehyde with an amine. This constitutes a great advantage because of the possibility to choose among several aldehydes or $\alpha$-amino acids as precursors that yield a variety of functional groups that can be grafted on graphene. For instance, Zang et al. decorated liquid-phase exfoliated graphene with palladium porphyrins by employing the porphyrin aldehyde and sarcosine as precursors. ${ }^{[78 \mathrm{~b}]}$ Another technique for the functionalization of pristine graphene that can afford a large variety of substituents relies on the use of organic azides. Their reactivity with the $C=C$ bonds in graphene is triggered by light or heat and goes through the formation of a reactive nitrene intermediate (Figure 9 b). For example, Liu et al. ${ }^{[73]}$ functionalized solvent-exfoliated 


\section{WILEY-VCH}

graphene flakes with different para-substituted perfluorophenylazide, using either heat or light irradiation (Figure 9 c). They also functionalized with the same procedure graphene immobilized in FET. They found out that in this case, the carrier mobility of functionalized graphene was doubled in comparison to pristine graphene and they did not observe a defective $\mathrm{D}$ peak in the Raman spectrum, suggesting that this reaction did not perturb the aromatic structure of graphene. This reaction was used also to functionalize graphene with a variety of alkyl azides which greatly increased the dispersibility of graphene in organic solvents, ${ }^{[79]}$ and to functionalize graphene with azidophenylalanine amino acids.${ }^{[80]}$ Cycloadditions to graphene can be realized via benzynes reactive intermediates that can be generated by removal of two ortho-substituents of benzene. For example, Zhong et al. covalently attached aryl rings to graphene using 2-(trimethylsilyl)aryl triate as benzyne precursor, greatly increasing the dispersibility of modified graphene in various solvents. ${ }^{[81]}$

a

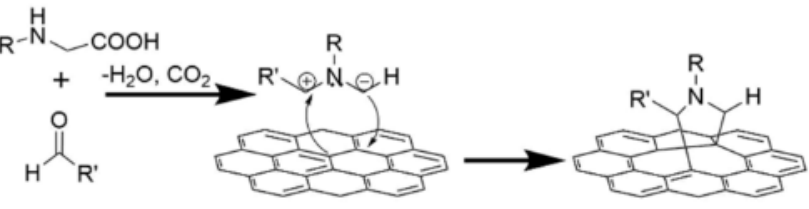

b

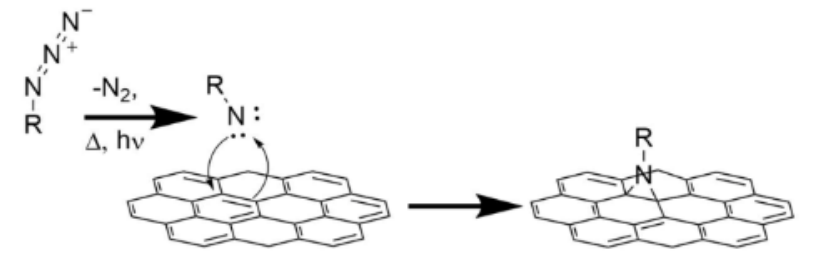

C

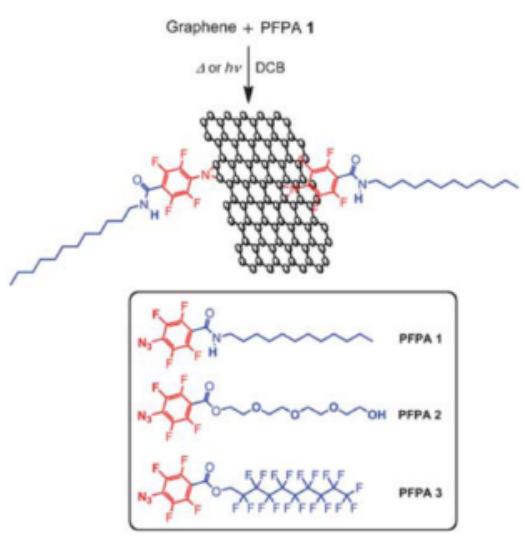

Figure 9: (a) Reaction scheme of the graphene functionalization with azomethine ylide. (b) Reaction scheme of the graphene functionalization with organic azides goes through formation of nitrene intermediate. (c) Solution-phase functionalization of pristine graphene with perfluorophenylazides. Adapted from Ref. ${ }^{[73]}$

Graphene oxide is a mono or few-layers graphitic material characterized by a random distribution of ordered $\mathrm{sp}_{2}$ carbon regions and disordered oxygen-rich regions with $\mathrm{sp}_{3}$ carbons. After the reduction of GO by chemical, thermal and microwave treatments most of the oxygens 


\section{WILEY-VCH}

are eliminated and the aromatic conjugation is restored. This causes the increase in electrical conductivity that ranges from around $1 \times 10^{-4} \mathrm{~S} / \mathrm{m}$ of GO up to $10^{2} \mathrm{~S} / \mathrm{m}$ of $\mathrm{rGO} .^{[11,82]}$ However, many defects remain in rGO, which are confirmed by its lower charge carrier mobility and electrical conductivity compared to pristine graphene, and by the persistence of a strong D band in the Raman spectrum of rGO. Because of this reason, when graphene is derived from the reduction of GO is usually referred to as reduced graphene oxide. The presence of hydroxyl, carboxyl and epoxy groups on GO allows a wide series of reactions and makes it the material of election for introducing functionalities on graphene through the covalent attachment of organic functional groups.

The carboxylic acids existing at the edges of GO flakes can be used for amide and esters formation with amines and alcohols. For example, Liu et al. prepared an oligothiophene functionalized GO exploiting the amide bond formation between an amine-terminated oligothiophene and the carboxylic groups at the edges of GO flakes (Figure 10 a). ${ }^{[83]}$ The hybrid material exhibited an improved absorption of light with optical limiting characteristics and efficient quenching of the photoluminescence of oligothiophene. Yu et al. ${ }^{[84]}$ functionalized GO flakes with regioregular poly(3-hexylthiophene) (P3HT) terminated with methanol groups through the formation of ester bonds with the carboxyl groups of GO. This material, which exhibited increased solubility in organic solvents, was used as active material in an organic solar cell displaying an increase of $200 \%$ in power conversion efficiency compared to the $\mathrm{P} 3 \mathrm{HT}$ alone. $\mathrm{Xu}$ et al. reported the covalent functionalization of GO via amide bond formation with porphyrins (Figure 10 b) ${ }^{[85]}$ The energy transfer efficiency from the porphyrin to GO was greatly enhanced by the covalent bond between them and the hybrid material exhibited better optical limiting properties compared to fullerene $\mathrm{C}_{60}$ and to the physical mixture of $\mathrm{GO}$ and the porphyrin. The reaction of carboxylic acids with amines and alcohols to form respectively 


\section{WILEY-VCH}

amides and esters are rather slow and reversible reactions and necessitate harsh reaction conditions such as high temperature and presence of dehydrating agents to accelerate the reaction and shift the equilibrium toward the products. For this reason, often the carboxylic acid is activated to a more reactive intermediate before the reaction with the mild nucleophile amine or alcohol. Such activation of the carboxylic acid becomes mandatory when GO needs to be functionalized with molecules sensitive to harsh conditions such as proteins. For instance, Shen et al. functionalized GO flakes with the protein bovine serum albumin via diimide activation of the carboxyl groups before amidation. ${ }^{[86]}$ The reaction consisted in two steps: firstly, the carboxyl groups on GO were activated by ethyl(dimethylaminopropyl) carbodiimide (EDAC), which in presence of N-hydroxysuccinimide (NHS) formed a stable ester. Secondly, the active ester, which is a good leaving group, reacted at room temperature with the amines of BSA forming an amide bond (Figure $10 \mathrm{c}$ ). It was proven that this synthetic path makes it possible to avoid the protein denaturation. The same diimide chemistry has been used to functionalize GO with DNA strands, and further exploited as specific receptors for DNA fragments. ${ }^{[87]}$
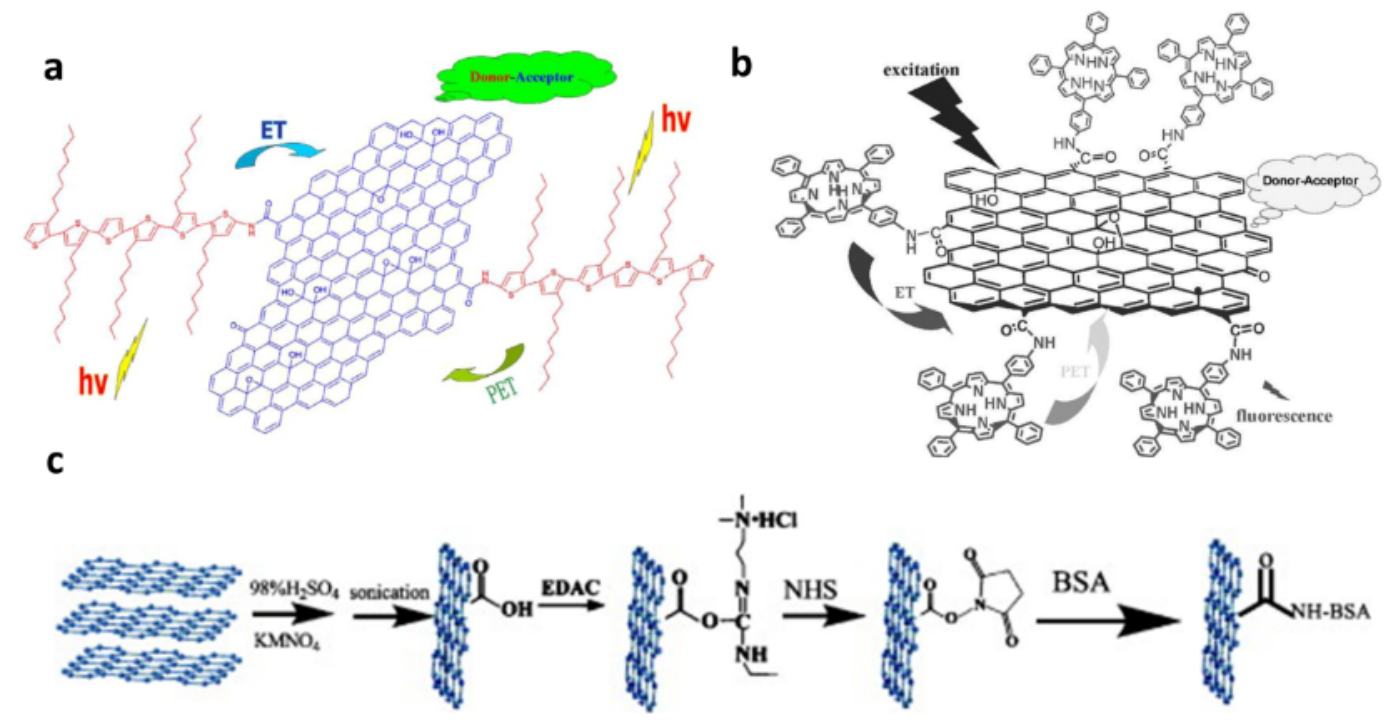

Figure 10: a) Structure of oligothiophene functionalized GO and energy transfer mechanism. Adapted from Ref. ${ }^{[83]}$ b) Schematic structure of the porphyrin functionalized GO and quenching mechanism. Adapted from Ref. ${ }^{[85]} \mathrm{c}$ ) Mechanism of the reaction between GO and BSA protein involving the use of a diimide intermediate. ${ }^{[86]}$ 


\section{WILEY-VCH}

GO can be grafted via amide and ester bond formation to polymers that bear hydroxyl and amine functional groups such as poly(vinyl alcohol (PVA), poly(ethylene glycol) (PEG), and polyallylamine. The properties of these hybrid materials are a combination of the properties of graphene such as mechanical strength and electrical conductivity with the properties of the polymeric part like dispersibility in certain solvents. For instance, GO can be made better dispersible in water by covalently modifying it with PEG chains. Liu et al. ${ }^{[88]}$ functionalized GO with PEG molecules terminating with amine groups by amide bond formation and used it as a deliverer for the water-insoluble cancer drug camptothecin. Camptothecin, which has an aromatic structure, grafts non-covalently via $\pi-\pi$ stacking to the GO flakes, which in turn is solubilized in water by the PEG chains. Salvagione et al. covalently tethered PVA with GO via ester bond formation. The ester could be formed either by Steglich esterification between the carboxylic acids of GO and PVA using dicyclohexylcarbodiimide as a coupling reagent or by firstly converting the carboxyl groups to the more reactive acyl chloride.

Another class of reaction that involves GO is the epoxide ring-opening reactions. The epoxide ring can be opened by nucleophiles such as amines and thiols in presence of basic or acid catalysis. Since GO is acidic for the presence of carboxyl groups and some sulphate groups, the reaction can take place by self-catalysis, without need for the addition of acids. ${ }^{[89]}$ The reaction between GO and amines or thiols can take place in mild conditions and have been exploited to attach a great range of amines and thiols to GO. ${ }^{[90]}$ Furthermore, it has been shown by Vacchi et al. that the reaction between GO and amines occurs via ring-opening of the epoxides preferentially to the amidation, since the first takes place in milder conditions and epoxides are more abundant than carboxyl groups in GO. ${ }^{[1]}$ The authors suggested that in the papers that describe the functionalization of GO by amide formations the functionalization of the epoxides 


\section{WILEY-VCH}

would have occurred more easily. Shan et al. ${ }^{[92]}$ described the functionalization of rGO with the biocompatible poly(l-lysine) via epoxide ring-opening. This modified rGO was soluble in biological media. After functionalization, the modified GO was used as an $\mathrm{H}_{2} \mathrm{O}_{2}$ efficient biosensor by firstly covalently attaching it to a gold electrode, then by immobilizing a peroxidase on the poly(l-lysine) chains (Figure 11 a). Compton et al. firstly functionalized the epoxide groups of GO flakes with hexylamine and then reduced the modified GO to modified rGO with hydrazine. ${ }^{[90]}$ This modified rGO exhibited a better solubility and processability compared to GO, while it displayed good electrical conductivity. Thomas et al. functionalized GO with potassium thioacetate via ring-opening of epoxide groups to form thiol-functionalized GO. ${ }^{[90 b]}$ This material showed high affinity for Au NPs that selectively deposited on its surface. The chemical modification of GO with specific functional groups is greatly exploited in the field of sensing. In fact, designed receptors can be grafted to GO in order to endow to this $2 \mathrm{D}$ material a specific sensitivity to target gases, molecules or biomolecules. ${ }^{[93]}$ In a recent study, Teradal et al. ${ }^{[93 \mathrm{c}]}$ assembled a capacitive-type porous graphene oxide (pGO) vapours sensor in which the GO was deposited on a pre-patterned electrode through a freeze-drying technique in order to maximize the area exposed to the gas. In parallel, the GO was also chemically functionalized with aniline (phenyl-GO), dodecylamine (dodecyl-GO) and ethanolamine (ethanol-GO) by amide bond formation: firstly the carboxylic acids of GO where converted to the acyl chloride with $\mathrm{SOCl}_{2}$ and then these were reacted with the amines (Figure $11 \mathrm{~b}$ ). These three differently functionalized GOs were deposited and combined with pGO in a sensor array that makes it possible to selectively detect the presence of water, $\mathrm{NH}_{3}$, toluene, $\mathrm{EtOH}$, phenol and cyclohexane (Figure $11 \mathrm{c}$ ). Chemically modified GO has been used also for metal ions sensing and removal. ${ }^{[94]}$ For instance, Seenivasan et al. ${ }^{[94 a]}$ used a cysteine functionalized $\mathrm{GO} /$ polypyrrole nanocomposite for $\mathrm{Pb}^{2+}$ ions detection and removal from water. The cysteine 


\section{WILEY-VCH}

functionalized GO (sGO) was synthesized in a two-steps process via amide and carbamate bond formation using carbonyldiimidazole as a sacrificial linker. Then the nanocomposite of sGO with polypyrrole was formed on a working electrode by electrochemical deposition. The concentration of $\mathrm{Pb}^{2+}$ ions was measured by anodic stripping of the modified electrode, and the sensor presented a limit of detection of $0.07 \mathrm{ppb}$.

a

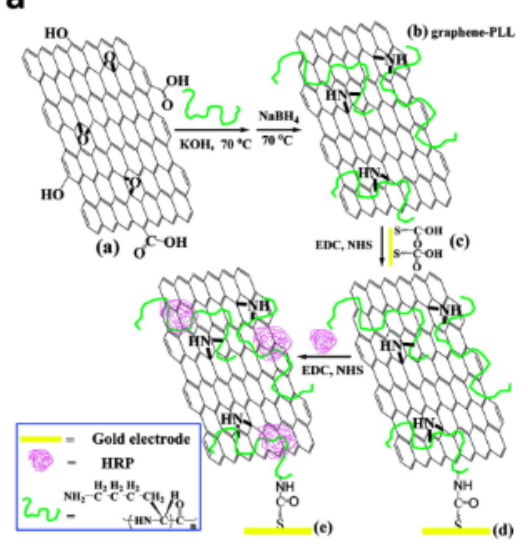

b

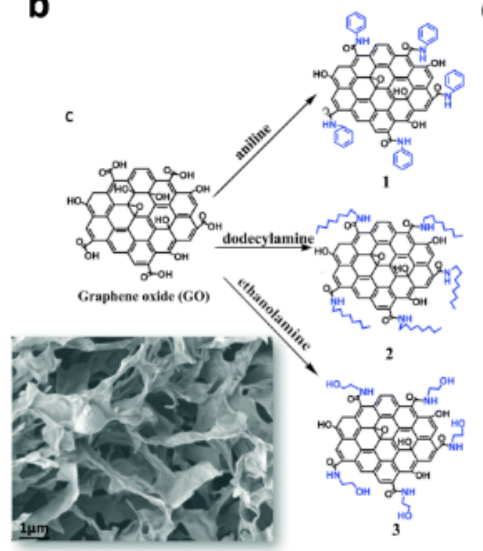

C

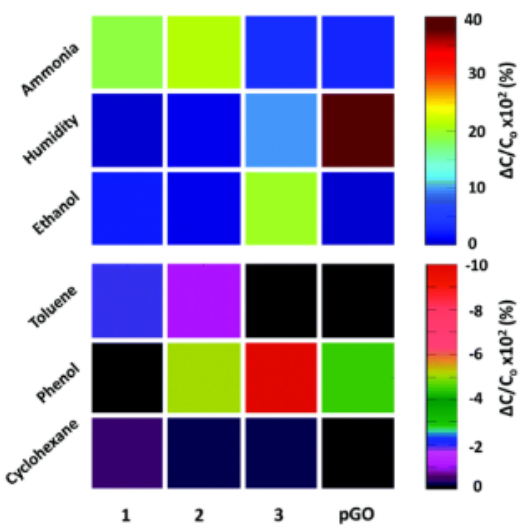

Figure 11: a) Schematic Diagram of GO-poly(1-lysine) synthesis and assembly process of modified GO at a gold electrode and following assembly with a peroxidase (HRP). Adapted from Ref. ${ }^{[92]}$ b) Schematic structure of the synthesis of phenyl-GO (1); dodecyl-GO (2); and ethanol-GO (3) and SEM image of the p-GO. c) Sensitivity to different vapours (180 ppm) using the functionalized pGO capacitive sensor: phenyl-GO (1); dodecyl-GO (2); and ethanolGO (3). The colours indicated in the diagram correspond to the sensitivity, according to the colour keys shown on the right. Adapted from Ref. ${ }^{[93 c]}$

Graphene oxide has also been covalently functionalized with polymers such as polystyrene (PS), poly(acrylamide) (PAM), poly(methyl methacrylate) (PMMA) using the atom transfer radical polymerization (ATRP) approach. ${ }^{[7,95]}$ The technique involves the covalent attachment of the initiator to GO, followed by the ATRP in presence of the monomers. Following this approach, Lee et al. ${ }^{[95]}$ synthesized GO sheets functionalized with PS, PMMA and poly(butyl acrylate). Firstly, GO was functionalized with the polymerization initiator $\alpha$-bromoisobutyryl bromide which reacted with the hydroxyl groups of GO by ester formation. Then the polymers were grown by suspending the functionalized GO in DMF in presence of the monomers and 


\section{WILEY-VCH}

copper as a catalyst. The obtained nanocomposites exhibited enhanced mechanical and thermal properties compared with the pure polymers. Similar mechanisms have been proposed to produce GO covalently grafted with PMMA and PS. ${ }^{[96]}$ In all the cases the composites were produced in two steps, by firstly covalently attaching the ATRP initiator to the functional groups of GO or directly on its basal plane, and secondly by growing the polymer on the functionalized GO. The resulting nanocomposites possessed higher dispersibility in organic solvents and improved mechanical properties such as an increase in the elastic modulus, hardness and strength. Free radical polymerization has been exploited also by Yang et al. ${ }^{[97]}$ to functionalize rGO with water-soluble poly(acrylamide) (PAM) and use it to adsorb $\mathrm{Pb}(\mathrm{II})$ and methylene blue from water (Figure 12 a). GO and rGO have been also functionalized with conductive polymers such as polypyrrole, polythiophene and polyaniline. These hybrid materials found mostly application as supercapacitors ${ }^{[98]}$ and solar cells. ${ }^{[84]}$ For example, in 2019 Li et al. synthesized an aerogel based on rGO grafted with polyaniline and tested it as a supercapacitor. ${ }^{[98 b]}$ Firstly amino groups were introduced on GO by conversions of the carboxylic groups to amides and attachment of benzamide molecules on the basal plane of GO via diazonium salt chemistry (Figure $12 \mathrm{~b}$ ). Then polyaniline was polymerized from the amino groups. The final hybrid material exhibited a maximum capacitance of $553 \mathrm{~F} \mathrm{~g}^{-1}$ in $1 \mathrm{M} \mathrm{H}_{2} \mathrm{SO}_{4}$.

a

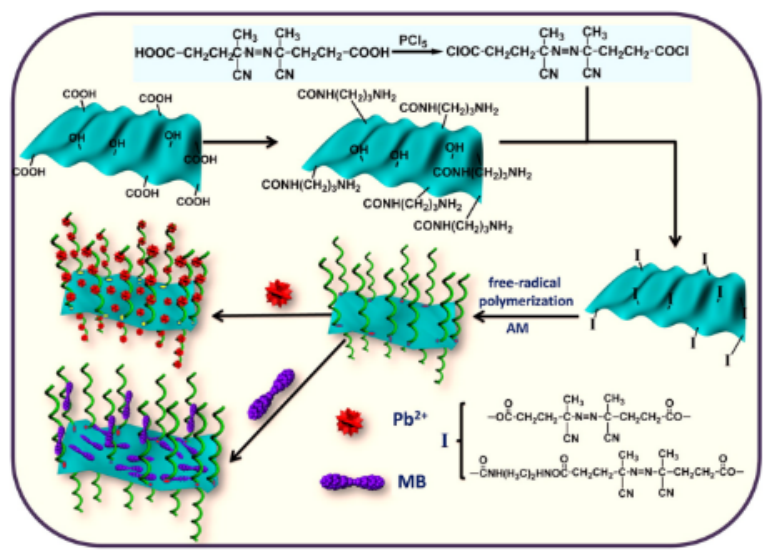

b

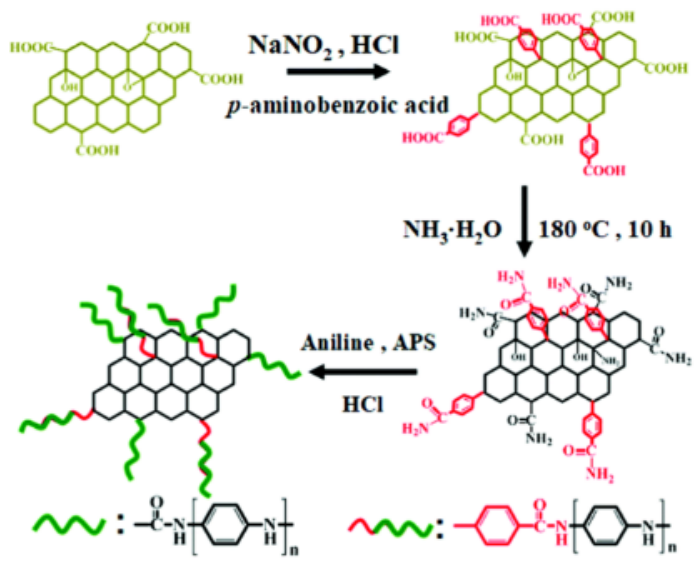




\section{WILEY-VCH}

Figure 12: a) Schematic of the synthesis of polyacrylamide functionalized rGO by free radical polymerization and adsorption of $\mathrm{Pb}(\mathrm{II})$ and Methylene Blue. Adapted from Ref. ${ }^{[97]} \mathrm{b}$ ) Schematic illustration showing the covalent functionalization of GO with polyaniline. Adapted from Ref. ${ }^{[98 b]}$

\subsubsection{Hybrid based on non-covalent interactions}

The majority of hybrid structures based on graphene with other molecules or nanostructures rely on non-covalent interaction rather than on covalent bonds. Non-covalent interactions rule the world around us; for instance, the presence of liquids is due to non-covalent interaction. Non-covalent interactions between molecules are the basis of supramolecular chemistry and determine the existence of biological systems, such as the DNA structure, the cell membrane and the 3D structure of proteins. Supramolecular/non-covalent bonds are usually reversible and kinetically labile, thus allowing for important mechanisms such as molecular recognition and self-correction. ${ }^{[99]}$ Among these forces we have ionic bonds, which are the strongest, with energies that range from 100-350 kJ/mol, followed by ion-dipole and coordinate (dative) bonds with energies between 50 and $200 \mathrm{~kJ} / \mathrm{mol}$, hydrogen bonds $(8-39 \mathrm{~kJ} / \mathrm{mol}), \pi-\pi$ and $\pi-\mathrm{CH}$ interactions $(5-30 \mathrm{~kJ} / \mathrm{ml})$ and lastly van der Waals interactions $(0.5-5 \mathrm{~kJ} / \mathrm{mol})$ (Figure 13). ${ }^{[16]}$ Pristine graphene may interact non-covalently with other molecules via $\pi$ - $\pi$ stacking and van der Waals forces, while GO and rGO in addition to these may also interact forming hydrogen, ligand-metal and ionic bonds through their oxygen functional groups. 


\section{WILEY-VCH}

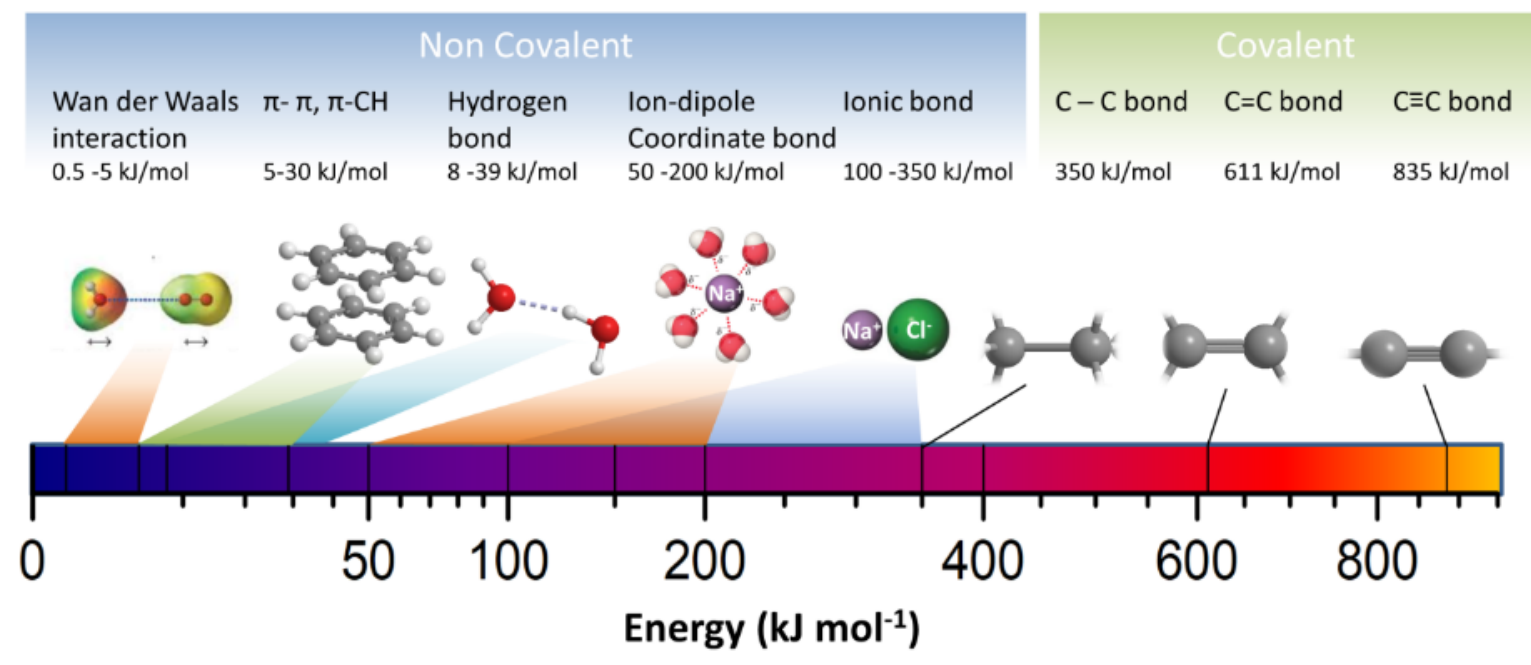

Figure 13: Schematic of the different covalent and non-covalent interactions with the corresponding energy ranges expressed in $\mathrm{kJ} / \mathrm{mol}$.

All forms of graphene, including its derivatives $\mathrm{GO}$ and $\mathrm{rGO}$, can be functionalized with aromatic molecules exploiting the $\pi-\pi$ interaction with its extended aromatic structure. The more extended are the two aromatic structures, the strongest is the resulting supramolecular bond. Besides, another requirement for the formation of $\pi-\pi$ interaction is the overlapping of the two $\pi$ systems, which is favoured by the planarity of the aromatic system. ${ }^{[16]}$ Experiments have shown that affinity and the binding constants between graphene materials and aromatic structures such as pyrenes follows the order $\mathrm{GO}<\mathrm{rGO}<$ pristine graphene, because of the increased aromaticity and planarity of the structure passing from GO to pristine graphene. ${ }^{[100]}$ The formation of $\pi-\pi$ stacking between aromatic molecules and graphene has been used to facilitate its exfoliation from graphite, increase its dispersibility in organic solvents and water, employed as a platform for drug delivery and form hybrid structures and nanocomposites with new advanced properties and functionalities. ${ }^{[16,74,101]}$ Molecules containing pyrene and perylenediimide (PDI) units are often used because of their strong interaction with graphene. In 2009 Su et al. ${ }^{[102]}$ functionalized rGO sheets with pyrene and PDI sulfonic acid derivatives. The resulting hybrids exhibited strong interaction between $\mathrm{rGO}$ and the two aromatic molecules 


\section{WILEY-VCH}

and were highly dispersible in water due to the presence of sulfonic groups attached to the pyrene and PDI, which induced repulsive forces between the hybrid nanostructures. Furthermore, they observed from the shift of the G band in Raman spectra the existence of a remarkable charge-transfer effect between the electron donor pyrene and rGO or between the electron acceptor PDI and rGO. The presence of the absorbed molecule was confirmed also by AFM imaging of the hybrid, which revealed an increased thickness of the rGO monolayer compatible with the height of the molecule. Pyrenes and other mall aromatic molecules decorated by lipophilic or hydrophilic have extensively been used to facilitate the ultrasoundinduced liquid-phase exfoliation (UILPE) of graphene. ${ }^{[16,103]}$ The small and flat aromatic molecules act as wedges: they penetrate between the layers of graphite with the aid of sonication and weaken the interactions that hold together the graphitic sheets by forming new competitive $\pi-\pi$ interaction with those. Eventually, the graphenic layers are completely dissociated and separated from each other; at this point the hydrophilic or lipophilic lateral chains that decorate the organic molecules aid to keep the graphene sheets dispersed in water or organic solvents respectively and to avoid the restacking between them. Palermo et al. ${ }^{[104]}$ studied the effects of the different functional groups decorating the aromatic molecule on the exfoliation and stabilization of graphene by UILPE. They employed four different pyrene molecules functionalized with one to four sulfonic groups, which are electron acceptors, while two of these molecules were also functionalized asymmetrically with one and two hydroxyl groups, which are electron donors (Figure 14 a). They find out that the more sulfonic groups were attached to the pyrene the less these molecules were adsorbed on graphite, while the exfoliation and stabilization in water were favoured by the molecule with the highest dipole moment, namely the one with two sulphonic and two hydroxyl groups. Pyranine modified rGO was used by Chen et al. ${ }^{[105]}$ to assemble a fast and highly sensitive humidity sensor. The hybrid was 


\section{WILEY-VCH}

produced in a one-pot procedure by contemporarily reducing GO with hydrazine and forming the non-covalent assembly with pyranine which assisted in stabilizing rGO in water (Figure 14 b). Qu et al. ${ }^{[106]}$ assembled a supramolecular hybrid of $\mathrm{rGO}$ and pyrene functionalized fullerene and emploied it as an electron accepting layer in an organic solar cell. The pyrene was used as an anchoring bridge to link $\mathrm{rGO}$ and the $\mathrm{C}_{60}$ fullerene. The performances of the cell with the hybrid material were greatly improved compared to the cell assembled with the single components.

a

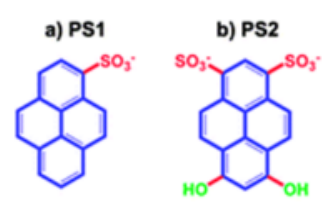

RATIO OF DYE CAPTURE ON GRAPHITE

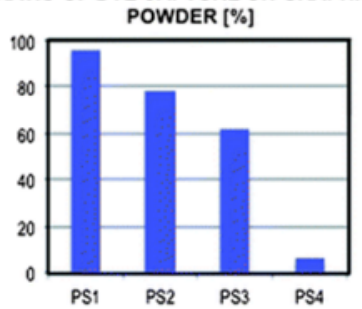

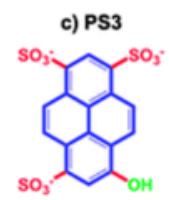

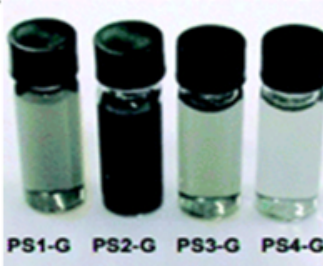

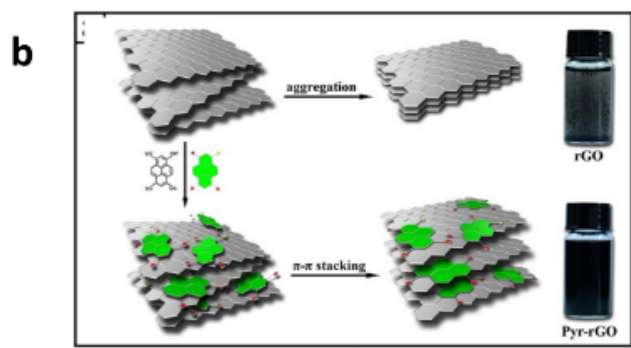
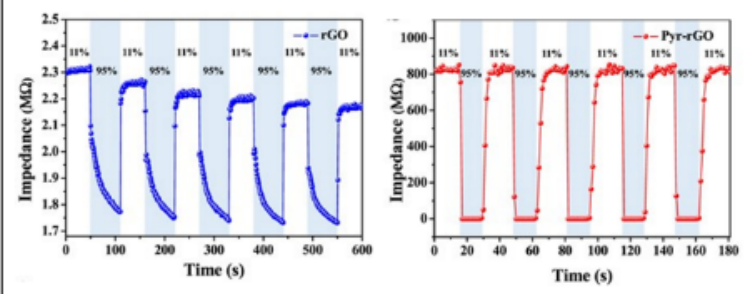

Figure 14: (a) Molecular structure of the 4 pyrene units modified with hydroxyl and sulfonic groups. Bottom left: the ratio of the molecules absorbed on graphite. Bottom right: Suspensions of UILP exfoliated graphene with the 4 dyes. Adapted from Ref. ${ }^{[104]}$ ( b) Stabilization of rGO by pyranine. Bottom: Impedance at $100 \mathrm{~Hz}$ of rGO (blue) and Pyranine-rGO (right) sensors under exposure to humidity between $11 \%$ and $95 \%$. Adapted from Ref. ${ }^{[105]}$

Aromatic electron-rich or electron-poor molecules adsorbed onto graphene can also modify its electrical properties. This can be seen in various theoretical papers revealing that molecules such as perylene-3,4,9,10-tetracarboxylic-3,4,9,10-diimide (PTCDI) can open a bandgap in pristine graphene. ${ }^{[107]}$ The use of molecular dopants interacting at the supramolecular level with graphene has the advantage to enable the tailoring of the electrical properties of graphene without the introduction of any structural defect or irreversible change of its form. In 2011 Zhang et al. ${ }^{[108]}$ showed that a 6 -fold increase of the $\mathrm{I}_{\mathrm{on}} / \mathrm{I}_{\text {off }}$ ratio of a FET based on mechanically 


\section{WILEY-VCH}

exfoliated bi-layer graphene could be achieved via the physisorption triazine molecules on the surface of the 2D material. The molecules were thermally evaporated and AFM characterization showed the formation of a uniform layer of molecules on the basal plane of graphene. An electrical bandgap up to $\sim 111 \mathrm{meV}$ at room temperature was estimated. ${ }^{[108]}$ Chen et al. ${ }^{[109]}$ studied the p-doping effect of the electron acceptor, tetrafluoro-tetracyanoquinodimethane (F4TCNQ) on epitaxial graphene grown on $\mathrm{SiC}(0001)$. By using synchrotron-based highresolution photoemission spectroscopy they revealed that the p-type doping of graphene is caused by the electron transfer from graphene to F4-TCNQ. Metallo-phthalocyanines (MPc) and porphyrins are other aromatic compounds that have been used to functionalize noncovalently graphene. In 2009 Gao et al. ${ }^{[10]}$ investigated the self-assembly of various magnetic phthalocyanines on epitaxial graphene monolayer by means of STM. They observed that the Pc self-assembled on the top of graphene by forming highly ordered supramolecular structures with a Kagome geometry that follow the lattice of epitaxial graphene yielding a Moiré pattern (Figure 15 a-b). Sainio and co-workers showed by STM that cobalt phthalocyanine selfassembled on epitaxial graphene grown on $\operatorname{Ir}(111)$ forming large and ordered domains with a square unit cell. ${ }^{[11]}$ The interaction between the delocalized $\pi$ system of graphene and the $\pi$ system of the adsorbed molecule can be so strong which can modify the structure of the latter. $\mathrm{Xu}$ et al. $^{[112]}$ demonstrated this rationale using a porphyrin substituted with methylpyridinium groups at the extremities (TMPyP). Normally the steric hindrance causes these groups to be aligned almost perpendicularly to the porphyrin plane. However, when they were absorbed on rGO the pyridinium groups were forced to align with the plane of the porphyrin to maximize the interaction with rGO. This was observed by a significant red-shift in the maximum of absorbance of the porphyrin (Figure $15 \mathrm{c}$-d). Furthermore, in the TMPyP/rGO assembly they observed a $100 \%$ quenching of the fluorescence of porphyrin by rGO and a huge acceleration 


\section{WILEY-VCH}

of the coordination reaction between $\mathrm{Cd}^{2+}$ ions and porphyrin that the group exploited to build a new optical probe for sensing $\mathrm{Cd}^{2+}$ ions. Jiang et al. ${ }^{[113]}$ created a water-dispersible selfassembled structure between a hydrophilic copper phthalocyanine (TSCuPc) and graphene sheets, by simply sonicating them together in water. The presence of the hybrid was pointed out by the UV-vis absorption that showed the disappearance of the band typical of the Pc dimer and the emergence of the band of the monomer, which was red-shifted because of the interaction with graphene. This hybrid was tested for photothermal and photodynamic therapy; after irradiating the hybrid dispersed in water at $650 \mathrm{~nm}$ for $10 \mathrm{~min}$ they observed a significant raise in the temperature of the suspension (Figure $15 \mathrm{e}$ ). Guo et al. ${ }^{[114]}$ used hemin, which is a Fe(III) porphyrin, to synthetize a hybrid with graphene sheets via $\pi-\pi$ interaction. The hybrid material exhibited interesting properties: it possessed intrinsic peroxidase-like activity and it was able to differentiate between single-strand and double-strand DNA owing to the different affinity of the hybrid towards the two DNA forms.
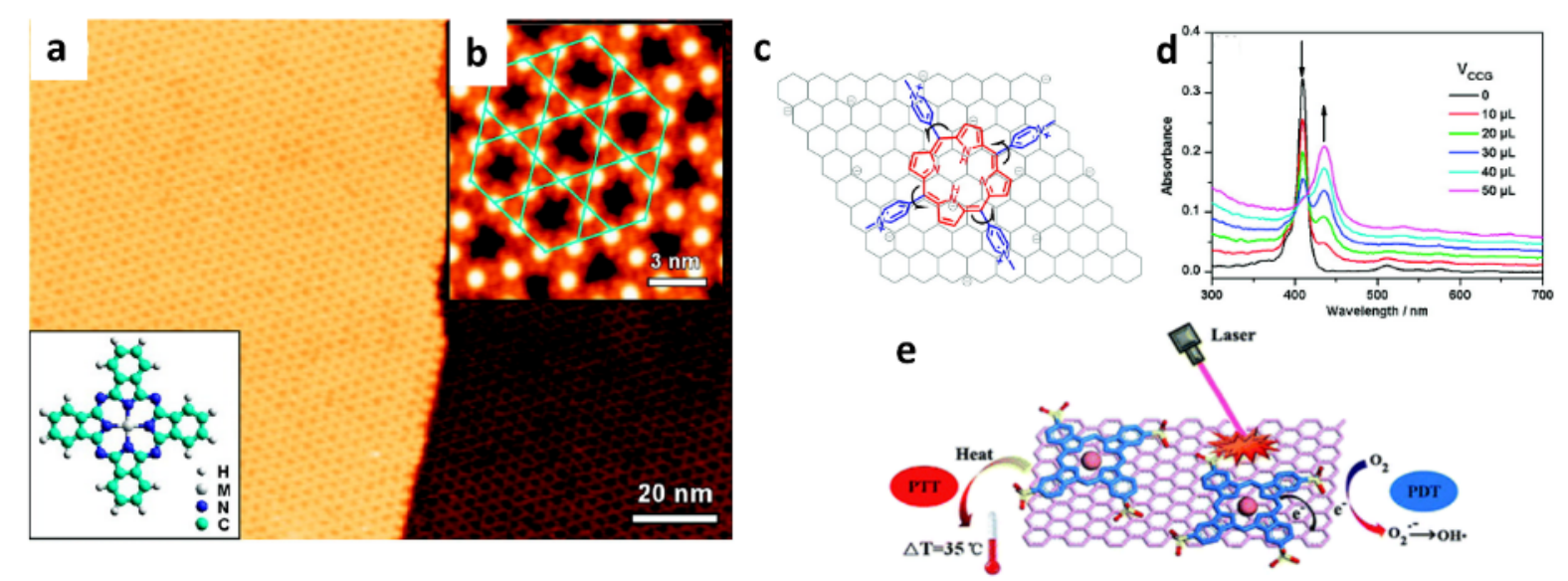

Figure 15: (a) STM images obtained of FePc adsorbed at RT on epitaxial graphene monolayer. The structural model of Pc is shown in the inset. (b) Magnified STM image showing details of the Kagome lattice of FePc. Adapted from Ref. ${ }^{[110]}$ (c) Schematic of the TMPyP adsorbed on graphene. (d) Absorption spectra recorded during the process of titration of an aqueous solution of TMPyP with rGO dispersion showing the red-shift in the absorbance peak of TMPyP. 


\section{WILEY-VCH}

Adapted from Ref. ${ }^{[12]}$ (e) Schematic showing the TsCuPc adsorbed on graphene and its use for photothermal (PTT) and photodynamic (PDT) therapy. Adapted from Ref. [113]

In addition to $\pi$ - $\pi$ stacking, non-aromatic molecules can adsorb onto graphene via van der Waals interactions; this is particularly true for alkyl chains and polymers whose collective interaction with graphene might be even stronger than the discussed $\pi-\pi$ interaction. ${ }^{[103]}$ Small molecules exposing alkyl lateral chains can form ordered self-assembled structure onto pristine graphene, and can also modify its electrical properties. ${ }^{[115]}$ De Feyter et al.$^{[116]}$ reported the formation of self-assembled monolayers (SAMs) of oleylamine (OA) on highly oriented pyrolytic graphite (HOPG) and epitaxial graphene on SiC. The detail of the supramolecular ordered structure was highlighted from AFM and STM images which revealed the formation of a lamellar structure in which the oleylamine molecules lie parallel one another forming a lamellar adlayer onto the basal plane of graphene. When the electrical characteristics of such hybrid structure was tested in back-gated FET, it was observed that oleylamine induced a tunable n-doping effect in graphene and at the same time did not cause any change in the electron and hole mobility. The same group studied also SAM of octadecylamine (ODA) and nonacosylamine (NCA) on HOPG and CVD graphene showing the formation of similarly ordered structure and a different ndoping level for the two molecules. ${ }^{[117]}$ In fact, the doping unit is the amine group, while the alkyl chains are responsible for the ordered self-assembly of the molecules. Since NCA has a longer alkyl chain than ODA, when NCA is assembled onto graphene it causes a lower density of amines on the surface, which ultimately leads to a lower doping effect, as it was confirmed by Raman spectroscopy and electrical measurements on FETs. Molecules composed by a photochromic or photoreactive head and a long aliphatic chain can form ordered molecular SAM on graphene and exhibit light-triggered dynamic doping effect, as it was recently demonstrated by our group. ${ }^{[118]}$ In 2017, Gobbi et al. ${ }^{[118 a]}$ verified the formation of selfassembled 2D supramolecular lattices on graphene using 3-trifluoromethyl-3-(3- 


\section{WILEY-VCH}

octadecyloxyphenyl)diazirine (MBB-1) (Figure 16 a). This molecule exhibited a photoreactive diazirine head group substituted with an electron-withdrawing $\mathrm{CF}_{3}$ moiety. Upon UV light irradiation of MBB-1 in chloroform, the photolysis of the diazirine unit produced a new molecule (MBB-2), exposing the $\mathrm{CF}_{3}$ group perpendicular to the molecular plane (Figure 16 b). Both molecules spin-coated on CVD graphene monolayers self-assembled forming ordered SAM exhibiting a lamellar structure. However, while MBB-1 did not exhibit a noticeable shift in the Dirac point of graphene, MBB-2 revealed a strong p-doping, with a positive shift in the Dirac point of more than $25 \mathrm{~V}$. The reason was attributed to a strong gating effect caused by the vertical orientation of the $\mathrm{CF}_{3}$ group in respect to the graphene plane in MBB-2, and thus by the high vertical dipole moment. In another work, Gobbi et al. studied the ordered SAM on graphene of a photochromic molecule consisting of a spiropyran head and a long aliphatic chain both on solid ${ }^{[118 b]}$ and at the solid-liquid interface. ${ }^{[118 c]}$ Spiropyran (SP) is a molecule that can reversibly isomerize to a metastable zwitterionic form with high dipole moment called merocyanine (MC) (structures in Figure 16 c). Both molecular forms were deposited on a graphene FET, on which they formed highly ordered lamellar structures. However, while the SP form had no doping effect on graphene, the MC form produced a high n-doping effect. By irradiating the supramolecular assembly with UV and visible light, it was possible to trigger the reversible isomerization of the molecules between the SP and MC forms, thereby yielding a reversible on-off switch of n-doping. The self-assembly of the same molecule on graphene was also studied at the solid-liquid interface. In this case only the MC form of the molecule was able to adsorb from solution on graphene forming an ordered lamellar SAM (Figure $16 \mathrm{c}$ ). By following the shift in the Dirac point of a graphene FET coated with a drop of the SP solution it was possible to monitor in real time the dynamic of the SAM formation caused by the isomerization of SP to MC upon irradiation with UV light (Figure $16 \mathrm{~d}-\mathrm{f}$ ). 


\section{WILEY-VCH}
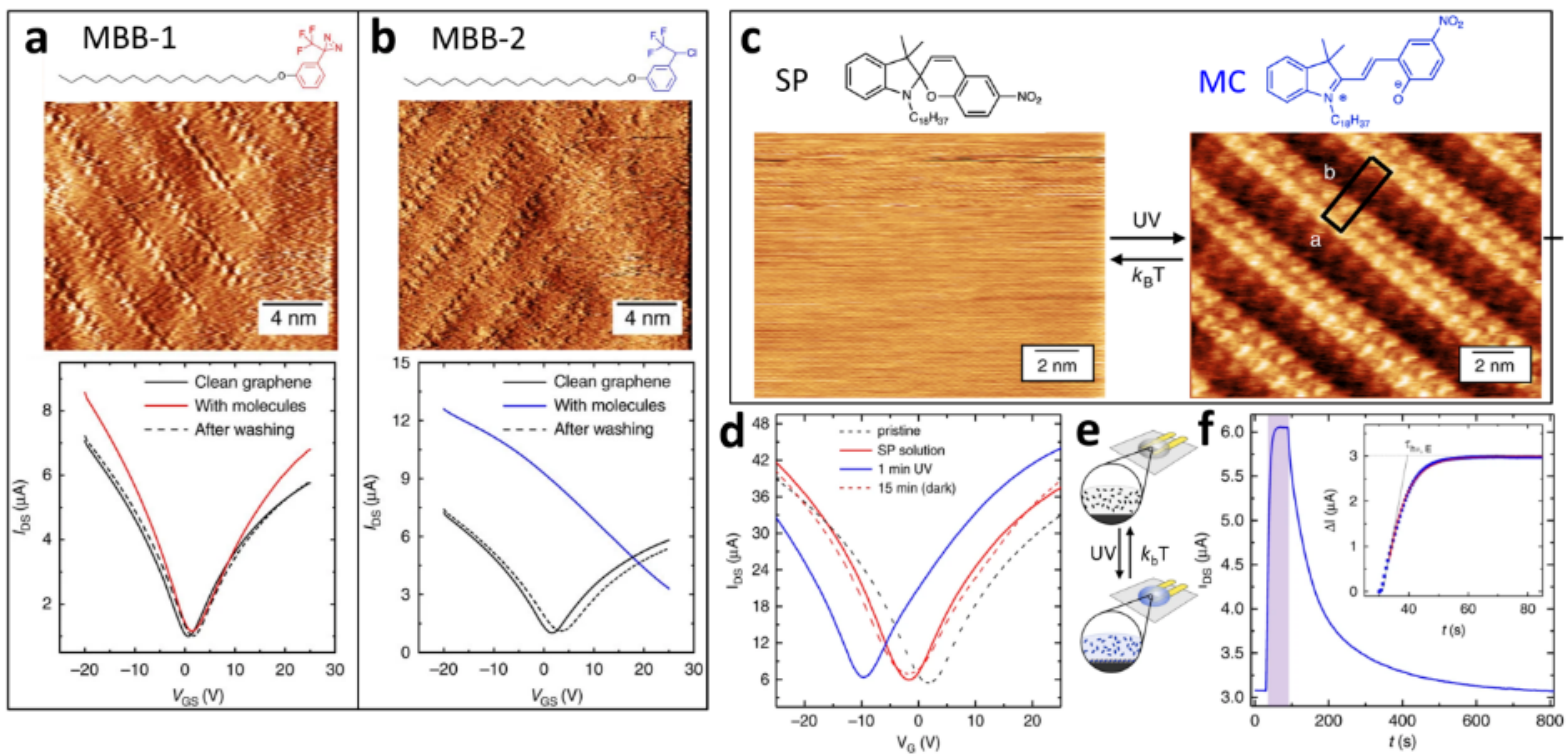

Figure 16: (a-b) Top panel: molecular structure of MBB-1 (a) and MBB-2 (b). Centre: STM image (current channel) of the SAM of (a) MBB-1 and (b) MBB-2 on CVD graphene. Lower panel: transfer curves of a graphene FET device before and after the deposition of (a) MBB-1 and (b) MBB-2. Adapted from Ref. ${ }^{[118 a]}$ (c) Molecular structure of SP and MC isomers and STM of SP (left) and MC (right) isomers assemblies at the solid-liquid interface between HOPG and a SP/MC solution. (d) Transfer curves of a graphene FET with a drop of SP solution on top as deposited, after UV irradiation (MC form), and after thermal relaxation (SP form). (e) Schematic of the isomerization between the two forms SP (black) and MC (blue) and selfassembly process on the graphene FET. (f) Dynamic monitoring of $\mathrm{I}_{\mathrm{DS}}\left(\right.$ at $\mathrm{V}_{\mathrm{GS}}=0 \mathrm{~V}$ ) before, during (in purple) and after UV irradiation. Adapted from Ref. ${ }^{[18 c]}$

Various other larger macromolecules such as polymers, including conductive ones like polyaniline, and biomolecules as DNA have been combined with graphene by tailoring their interactions primarily via van der Waals forces, albeit other kinds of interactions could also be present. Assemblies of these macromolecules with graphene have been extensively studied by targeting at diverse applications in many different fields. ${ }^{[16,74,119]}$ For instance, Choi et al. ${ }^{[120]}$ reported a free-standing flexible conductive $\mathrm{rGO} / \mathrm{Nafion}$ hybrid which was employed for the electrochemical sensing of organophosphate. The hybrid was formed by self-assembly of Nafion and rGO in solution, which was driven by the van der Waals interaction and hydrophobic effect between the fluorinated alkyl chain of Nafion and rGO. The hydrophilic sulphonated part of Nafion guaranteed to the hybrid a high dispersibility in water. After filtering 


\section{WILEY-VCH}

the resulting conductive hybrid paper was used as biosensing platform for organophosphate with high sensitivity and detection limit of $1.37 \times 10^{-7} \mathrm{M}$. Bai et al. ${ }^{[121]}$ produced a hybrid of rGO and sulfonated polyaniline (SPANI), by reducing GO in presence of SPANI. The hybrid was stably dispersed in water and after deposition on a glassy carbon electrode showed improved electrochemical stability and enhanced electrocatalytic activity. Wang et al. ${ }^{[122]}$ fabricated a graphene/PANI hybrid paper via in-situ electropolymerization of PANI. The flexible and mechanically resistant hybrid paper was tested as a supercapacitor and exhibited a capacitance of $233 \mathrm{~F} \mathrm{~g}^{-1}$ and $135 \mathrm{~F} \mathrm{~cm}^{-3}$. DNA has been used extensively to decorate graphene non-covalently. ${ }^{[119]}$ DNA can interact with graphene and GO via van der Waals, $\pi-\pi$ interactions and hydrogen bond formations. In 2009 Mann et al. ${ }^{[123]}$ presented one of the first examples of hybrid DNA-rGO. They showed that the reduction of GO in presence of single strand DNA produced a hybrid structure which is highly dispersible in water, that self-assembled forming layered assembly after solvent evaporation (Figure 17 a). Liu et al. ${ }^{[124]}$ reported a rGO/DNA/AuNPs hybrid structure. DNA strands terminated with a thiol group were selfassembled onto GO flakes, which were then reduced with hydrazine. The resulting hybrid rGO/DNA dispersions were highly stable in water and were used to selectively adsorb AuNPs thanks to the thiol groups, forming the hybrid rGO/DNA/AuNPs (Figure 17 b-c). In $2010 \mathrm{Xu}$ et al. ${ }^{[125]}$ discovered that GO and DNA could self-assemble yielding a stable hydrogel with high mechanical strength and environmental stability, high dye-adsorption capacity, and self-healing function. The hydrogel structure was formed by heating a water suspension of GO and double strand DNA: upon heating the DNA unwounded into a single strand form, cross-linking together the GO flakes via strong non-covalent interactions (Figure $17 \mathrm{~d}-\mathrm{f}$ ). 


\section{WILEY-VCH}

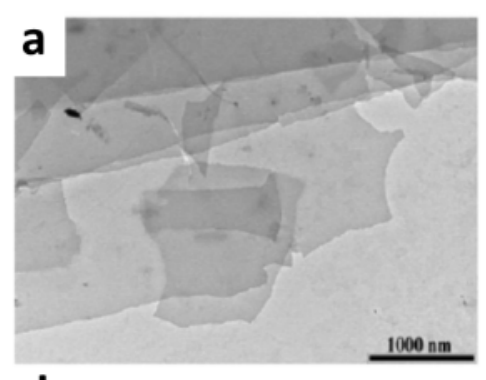

d

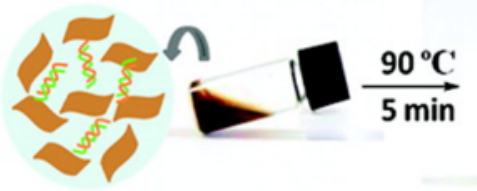

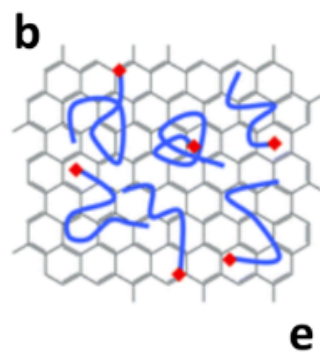
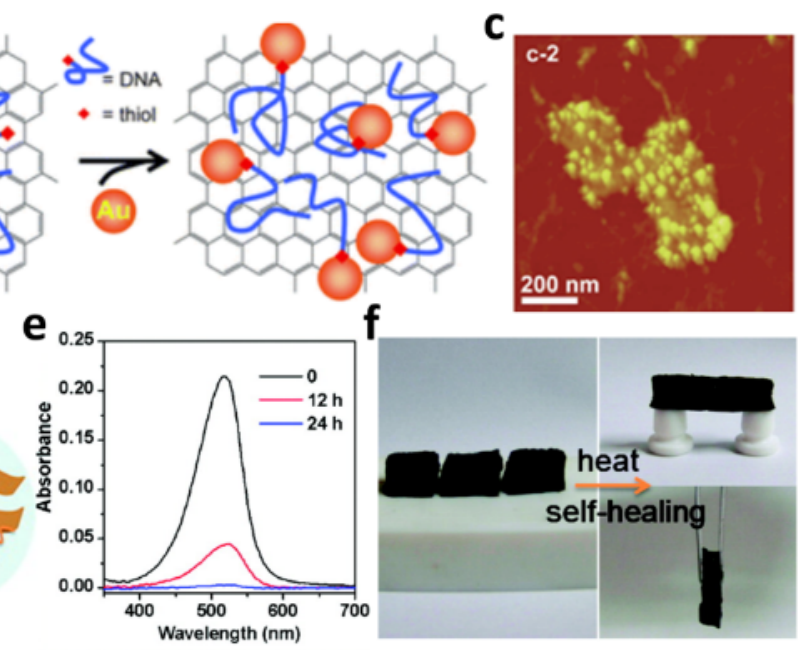

Figure 17: (a) TEM image of layered structure rGO-DNA. Adapted from Ref. ${ }^{[123]}$ (b) Schematic of the self-assembled hybrid rGO/DNA-SH and selective adsorption of Au NPs. (c) AFM image of rGO/DNA-SH/Au NPs hybrid. Adapted from Ref. ${ }^{[124]}$ (d) Schematic procedure of preparing GO/DNA self-assembled hydrogel and the proposed gelation mechanism. Absorption spectra of an aqueous solution of safranin-O after adsorption by GO/DNA hydrogel and (f) self-healing capability of the hydrogel. Adapted from Ref. ${ }^{[125]}$

As previously discussed, GO exposes several oxygen-containing functional groups, including carboxylic groups which donate to GO a negative charge, in fact, GO can be considered an anionic polyelectrolyte. For this reason, GO can form self-assembled structures via hydrogen bonds, yet more importantly via electrostatic and ionic interactions. These considerations are partially true also for rGO, which conserves part of the oxygen functionalities and thus part of the negative charge. Müllen and co-worker firstly exploited this effect in 2009 to assemble noncovalently functionalized GO and rGO dispersible in organic solvents such as chloroform ${ }^{[126]}$. They mixed a GO water suspension with positively charged quaternary ammonium salts with long alkyl chains. The amphiphilic molecules were self-assembled on the GO and rGO surface with the hydrophobic part oriented towards the water phase. Upon mixing the suspension with chloroform the hybrid was transferred to the organic phase (Figure 18 a). The electrostatic interactions between $\mathrm{GO} / \mathrm{rGO}$ and positively charged molecules have been extensively used to build self-assembled layer-by-layer (LbL) structures for disparate applications. Zhao et al. ${ }^{[127]}$ 


\section{WILEY-VCH}

reported a self-assembled LbL structure of alternating GO and PVA, obtained by soaking cyclically a substrate in a PVA and GO solution. The assembly in a layered structure was driven by the formation of hydrogen bonds between GO and PVA (Figure 18 b). The composite exhibited a $98.7 \%$ improvement of elastic modulus and a $240.4 \%$ increase of hardness compared to PVA. Hu and Mi presented a membrane for forward osmosis composed by a LbL assembly via electrostatic interactions between GO and the positively charged poly(allylamine hydrochloride). ${ }^{[128]}$ In 2014 Zou and $\mathrm{Kim}^{[129]}$ showed that the positively charged branched polyethyleneimine (PEI) dripped into a water suspension of GO induced the self-assembly of the latter in porous layered structures. The LbL assembly was driven by diffusion of PEI through the porous structure and by the electrostatic interaction between the two components and therefore could progress without additional external forces or stimuli. By adjusting the concentration of GO the researchers were able to obtain hollow spheres composed by the LbL assembly with diameters of several $\mathrm{mm}$, that once analysed at SEM revealed a porous layered structure (Figure $18 \mathrm{c}-\mathrm{d}$ ). These beads showed efficacy in absorbing oil and exhibited electrical conductivity once reduced. LbL structures have also been employed for sensing. For example, Zhang et al. ${ }^{[130]}$ fabricated a humidity sensor based on the LbL assembly of GO and the cationic polyelectrolyte poly(diallyldimethylammonium chloride) (PDDA). The nanostructured film was fabricated by immersing alternatively a substrate into PDDA and GO suspensions for five repetitive cycles, after which it was chemically reduced. The assembled device exhibited a 37\% response passing from $0 \% \mathrm{RH}$ to $97 \% \mathrm{RH}$ and long-term stability over time. 


\section{WILEY-VCH}
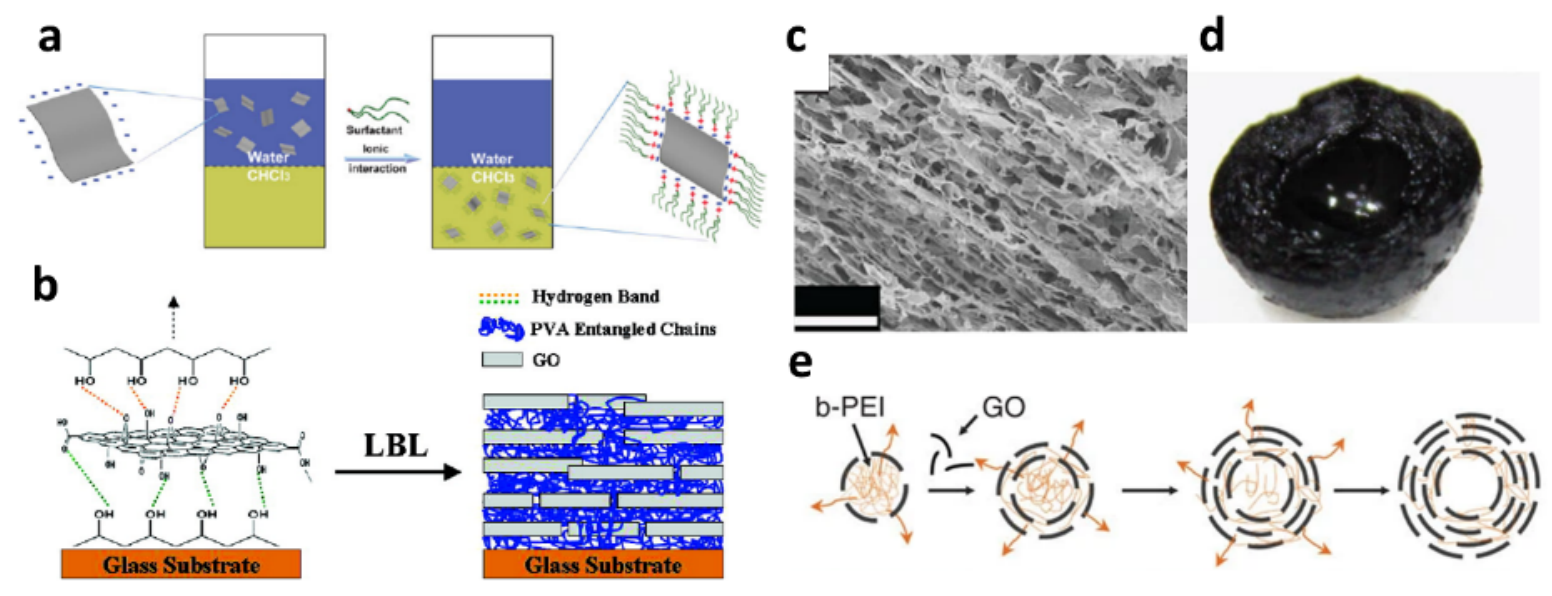

Figure 18: (a) Schematic of the self-assembly between negatively charged GO and positively charged quaternary ammonium salts, that make it soluble in chloroform. Adapted from Ref. ${ }^{[126]}$ (b) Schematic of the LbL assembly between GO and PVA. Adapted from Ref. ${ }^{[127]}$ (c) SEM image and (d) photograph of the self-assembled LbL GO/PEI porous layered structure and (e) schematic of the spontaneous formation of the structure. Adapted from Ref. [129]

\section{Application of hybrid assembly based on graphene}

Graphene possesses some outstanding characteristics such as the enormous surface to volume ratio, high electrical and thermal conductivity, and mechanical strength. Its combination with organic molecules or inorganic nanostructures is a powerful strategy to impart to such a unique 2D material some additional functionalities, yielding functional hybrids which can find applications in many different fields, including sensing of molecules and biosensing, ${ }^{[27 \mathrm{a}]}$ water purification and removal of contaminants, ${ }^{[27 a, 131]}$ (photo)catalysis and water splitting, ${ }^{[132]}$ biomedical application, ${ }^{[133]}$ energy storage, ${ }^{[36 a, 36 d, 56 b, 134]}$ photodetectors ${ }^{[135]}$ and transparent electrodes for optoelectronics. ${ }^{[135]}$ In Table 1 is presented a summary of the major applications of graphene-based hybrids, with the performances of the state of the art benchmarked to the current commercial technologies. In the following section, the applications of such hybrids are presented and discussed in detail. 
Table 1: Major applications of graphene-based hybrid materials: state of the art, potentials and drawbacks compared to commercial technologies.

\begin{tabular}{|c|c|c|c|c|}
\hline $\begin{array}{l}\text { Field of } \\
\text { application }\end{array}$ & $\begin{array}{l}\text { State of the art of } \\
\text { commercial } \\
\text { technologies }\end{array}$ & $\begin{array}{l}\text { State of the art of } \\
\text { graphene hybrid }\end{array}$ & $\begin{array}{l}\text { Potentials/advantages } \\
\text { of graphene hybrid }\end{array}$ & Challenges/drawbacks \\
\hline $\begin{array}{l}\text { Water purification } \\
\text { (Reverse } \\
\text { osmosis } \\
\text { membrane) }\end{array}$ & $\begin{array}{l}\text { Polyamide Thin-Film } \\
\text { Composite (duPont } \\
\text { FilmTec }{ }^{\mathrm{TM}} \text { SW30HR) } \\
\text { NaCL rejection: } 99.6 \% \\
\text { Flow rate: } 950 \mathrm{~L} \mathrm{~h}^{-1}\end{array}$ & $\begin{array}{l}\text { GO-FLG-deoxycholate } \\
\text { membrane } \\
\text { NaCl rejection: } 85 \% \\
\text { Flow rate: } 60 \mathrm{~L} \mathrm{~h}^{-1}\end{array}$ & $\begin{array}{l}\text { Nanoporous graphene: } \\
\text { predicted } \mathrm{NaCl} \text { rejection } \\
\text { of } 100 \% \text { at } 5 \mathrm{~L} \mathrm{~h}^{-1} \mathrm{~cm}^{-2} \\
\mathrm{MPa}^{-1}[137]\end{array}$ & $\begin{array}{l}\text { Scale up graphene } \\
\text { dimensions while } \\
\text { maintaining } \\
\text { mechanical properties }\end{array}$ \\
\hline $\begin{array}{l}\text { Gas sensing } \\
\left(\mathrm{NO}_{2} \text { sensor }\right)\end{array}$ & $\begin{array}{l}\text { Alphasense NO2-B43F } \\
\text { l.o.d. = } 40 \text { ppb linear } \\
\text { response up to } 5 \text { ppm }\end{array}$ & $\begin{array}{l}\mathrm{rGO}-\mathrm{In}_{2} \mathrm{O}_{3} \\
\text { nanocrystals hybrid } \\
\text { [138] } \\
\text { I.o.d. = } 10 \mathrm{ppb} \text { linear } \\
\text { response up to } 1 \mathrm{ppm}\end{array}$ & $\begin{array}{l}\text { Lower cost of rGO and } \\
\text { GO based sensors, } \\
\text { lower I.o.d. }\end{array}$ & $\begin{array}{l}\text { Long term stability and } \\
\text { reproducibility }\end{array}$ \\
\hline $\begin{array}{l}\text { Metal sensing } \\
\text { (Hg sensing) }\end{array}$ & $\begin{array}{l}\text { CV-AFS } \\
\text { I.o.d. }=2 \times 10^{-6} \mathrm{ppm}^{[139]}\end{array}$ & $\begin{array}{l}\text { CVD graphene- } \\
\text { aptamer hybrid }{ }^{[140]} \\
\text { l.o.d. }=2 \times 10^{-7} \mathrm{ppm} \text {, } \\
\text { high selectivity }\end{array}$ & $\begin{array}{l}\text { Lower cost and } \\
\text { portability of the } \\
\text { graphene-based } \\
\text { sensor. Lower I.o.d. }\end{array}$ & $\begin{array}{l}\text { Long term stability and } \\
\text { reproducibility }\end{array}$ \\
\hline $\begin{array}{l}\text { Photocatalysis } \\
\text { and } \\
\text { electrocatalysis } \\
\text { (electrolytic HER) }\end{array}$ & $\begin{array}{l}\text { PEM electrolysis } \\
\text { Efficiency up to } 80 \% \\
\text { [141] }\end{array}$ & $\begin{array}{l}\mathrm{rGO} / \mathrm{MoS}_{2} \text { hybrids } \\
\text { Tafel slope }=4 \mathrm{mV} \\
\mathrm{dec}^{-1} \text { overpotential }= \\
0.1 \mathrm{~V}\end{array}$ & $\begin{array}{l}\text { Lower cost of the rGO } \\
\text { based hybrid compared } \\
\text { to the expensive Pt and } \\
\text { Ir based catalysts used } \\
\text { in commercial PEM }\end{array}$ & $\begin{array}{l}\text { Performances still } \\
\text { lower than commercial } \\
\text { Pt/Ir catalysts }\end{array}$ \\
\hline $\begin{array}{l}\text { Energy storage } \\
\text { (lithium ion } \\
\text { batteries) }\end{array}$ & $\begin{array}{l}\text { Panasonic } \\
\text { NCR18650GA } \\
\text { Energy density = } 224 \\
\mathrm{mWh} \mathrm{g}^{-1} \text { (a) } \\
64 \% \text { capacitance } \\
\text { retention after } 500 \\
\text { cycles }\end{array}$ & $\begin{array}{l}\mathrm{N} \text {-doped rGO / } \mathrm{SnO}_{2} \\
\mathrm{NPs} \text { hybrid }{ }^{[134 \mathrm{~b}]} \\
\text { Energy density }=4049 \\
\mathrm{mWh} \mathrm{g}^{-1}(\mathrm{~b}) \\
99 \% \text { capacitance } \\
\text { retention after } 500 \\
\text { cycles }\end{array}$ & $\begin{array}{l}\text { Higher power densities } \\
\text { and energy densities, } \\
\text { longer lifetime and } \\
\text { cyclability }\end{array}$ & Reduction of cost \\
\hline $\begin{array}{l}\text { Energy storage } \\
\text { (super capacitor) }\end{array}$ & $\begin{array}{l}\text { Maxwell PCAP0300 } \\
\text { pseudocapacitor } \\
\text { Specific energy = } \\
0.0091 \mathrm{Wh} \mathrm{g}^{-1}(\mathrm{a}) \\
\text { Gravimetric capacitance } \\
=12.5 \mathrm{~F} \mathrm{~g}^{-1} \text { (a) }\end{array}$ & $\begin{array}{l}\text { rGO decorated with } \\
\mathrm{Ni}_{3} \mathrm{~S}_{2} \mathrm{NPs}^{[142]} \\
\text { Specific energy }= \\
0.071 \mathrm{Wh} \mathrm{g}^{-1}{ }^{(\mathrm{b})} \\
\text { Gravimetric } \\
\text { capacitance }=1424 \mathrm{~F} \\
\mathrm{~g}^{-1(\mathrm{~b})}\end{array}$ & $\begin{array}{l}\text { Higher specific energy } \\
\text { and gravimetric } \\
\text { capacitance }\end{array}$ & $\begin{array}{l}\text { Improve durability and } \\
\text { reduction of cost }\end{array}$ \\
\hline $\begin{array}{l}\text { Transparent } \\
\text { electrodes }\end{array}$ & $\begin{array}{l}\text { ITO Glass Substrates } \\
\mathrm{T}_{(550 \mathrm{~nm})}=84 \% \mathrm{R}_{\mathrm{s}}=12 \\
\Omega \mathrm{sq}^{-1}\end{array}$ & $\begin{array}{l}\text { Ag NWs / CVD } \\
\text { graphene on PC }{ }^{[43 b]} \\
\mathrm{T}_{(550 \mathrm{~nm})}=88 \% \mathrm{R}_{\mathrm{s}}=8 \\
\Omega \mathrm{sq}^{-1}\end{array}$ & $\begin{array}{l}\text { Lower sheet resistance } \\
\text { and higher } \\
\text { transparency compared } \\
\text { to ITO and potentially } \\
\text { cheaper }\end{array}$ & $\begin{array}{l}\text { Prices still high and } \\
\text { low long term stability } \\
\text { and robustness }\end{array}$ \\
\hline
\end{tabular}

CV-AFS: cold vapour atomic fluorescence spectroscopy. PEM: polymer electrolyte membrane. 1.o.d.: limit of detection. FLG: few layers graphene. (a): calculated over the entire cell weight (except casing). (b): calculated over the electrode weight.

\subsection{Sensing materials}

One important field of application of graphene-based hybrid materials is chemical sensing of small molecules, biomolecules and metal ions as well as sensing of electromagnetic radiations (i.e. photodetectors) and sensing of other physical properties (e.g. temperature and pressure). Most of the sensing devices have an electrical output. In other words, the quantity that is measured is commuted to a change in a measurable electrical property. The performances of a chemical sensor are characterized by different figures of merit, among which the most important 


\section{WILEY-VCH}

are the sensitivity, selectivity, limit of detection, hysteresis, and response time. The main advantages of using graphene as a chemical sensor with an electrical readout are its high conductivity, its low intrinsic electrical noise, ${ }^{[143]}$ that allows the detection of minimal changes in the charge carrier density or electrical resistance, and its vast surface area, that makes it possible to maximize the exposure to the analyte. ${ }^{[27 a]}$ Graphene, GO and rGO can be combined with molecules or nanostructures that act as specific receptors capable to interact with the sensed analyte as a route to increase its sensitivity and selectivity toward the measured quantity. Besides, the hybrids based on graphene typically exhibit significantly increased porosity compared to films of pristine graphene, which results in an enhanced accessible surface area.

\subsubsection{Chemical sensing}

Gas sensors play an important role in many industrial sectors, in environmental monitoring and personal safety. In an effort to improve the sensitivity and selectivity of gas sensors and reduce their consumption and cost, graphene-based hybrid materials are emerging as alternatives to current technological applications based mainly on metal oxides and inorganic semiconductors. ${ }^{[144]}$ For example, metallic nanoparticles (NPs) can enhance the sensitivity and selectivity to the sensed analytes due to specific catalytic effects. ${ }^{[27 a]}$ Li et al. ${ }^{[145]}$ fabricated a NO sensor based on reduced GO decorated with Pd NPs. This device exhibited high sensitivity with an impressive limit of detection of $2 \mathrm{ppb}$, which was remarkably higher than that of a reference device assembled without functionalized rGO. GO decorated with Pt NPs ${ }^{[25 b]}$ and CVD graphene decorated with Pd NPs ${ }^{[25 c]}$ have been used to sense hydrogen gas, by taking advantage of the high affinity of hydrogen towards these noble metals. An assembly of sulfonated rGO and Ag NPs was used to Huang et al. to sense $\mathrm{NO}_{2}$. ${ }^{[146]}$ The Ag NPs were reduced in situ on the rGO surface (Figure 19 a-b). This sensor displayed a $74.6 \%$ response to 50 ppm of $\mathrm{NO}_{2}$ (Figure 19 c). GO and rGO decorated with metal oxides such as $\mathrm{ZnO}, \mathrm{SnO}_{2}$, 


\section{WILEY-VCH}

$\mathrm{In}_{2} \mathrm{O}_{3}$, and $\mathrm{Cu}_{2} \mathrm{O}$ nanoparticles, nanorods and nanocrystals have also been used in the sensing of gases such as $\mathrm{NO}_{2},{ }^{[138,147]} \mathrm{NH}_{3},{ }^{[26 b]} \mathrm{H}_{2} \mathrm{~S},{ }^{[26 a, 41]} \mathrm{CO}^{[26 b]}$. For example, Liu et al. ${ }^{[138]}$ fabricated a hybrid of rGO and flower-like $\mathrm{In}_{2} \mathrm{O}_{3}$ nanocrystals via a facile one-step hydrothermal method and used it to sense $\mathrm{NO}_{2}$ (Figure $19 \mathrm{~d}$ ). This rGO- $\mathrm{In}_{2} \mathrm{O}_{3}$ composite showed enhanced sensing performance towards $\mathrm{NO}_{2}$ in comparison with the pure $\operatorname{In}_{2} \mathrm{O}_{3}$ sample, with a limit of detection of $10 \mathrm{ppb}$ (Figure 19 e).
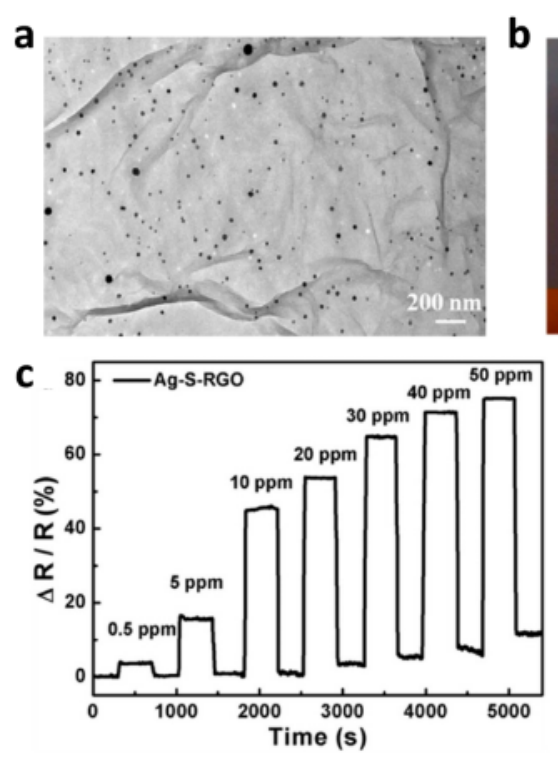

b

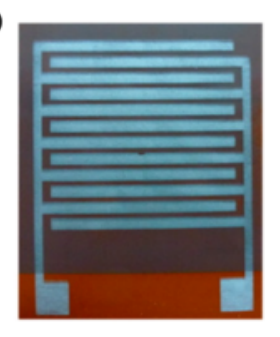

e d
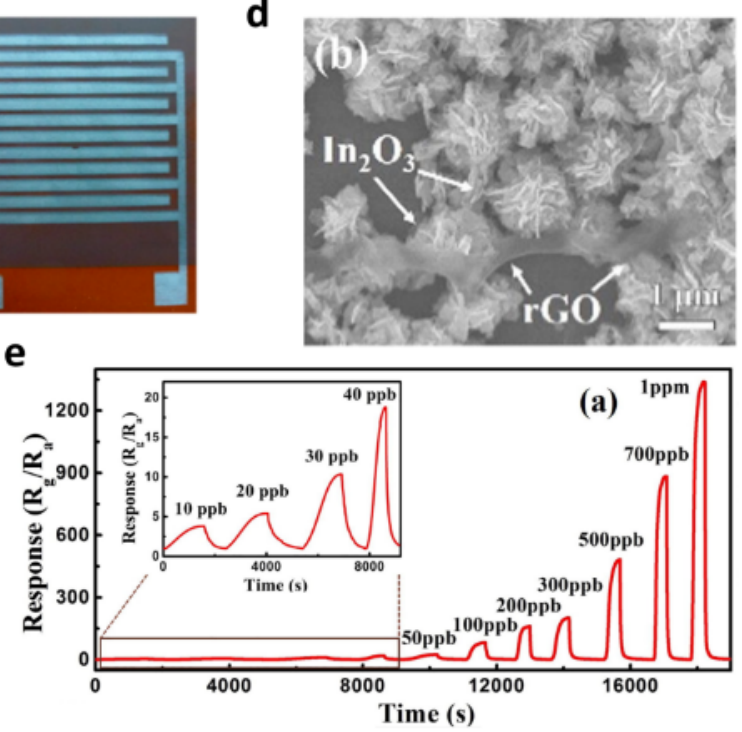

Figure 19: (a) Transmission electron microscopy (TEM) image of sulfonated rGO/Ag NPs and (b) photograph of the sensing device. (c) Response of the s-rGO/Ag NPs sensor as a function of time in various concentrations of $\mathrm{NO}_{2}$ gas. Adapted from Ref. ${ }^{[146]}$ (d) $\mathrm{SEM}$ of rGO- $\mathrm{In}_{2} \mathrm{O}_{3}$ hybrid. e) Response of the rGO- $\operatorname{In}_{2} \mathrm{O}_{3}$ hybrid to various concentrations of $\mathrm{NO}_{2}$. Adapted from Ref. [138]

In order to target specifically the sensed gases, GO and rGO have also been functionalized covalently or non-covalently with polymers and molecules exhibiting specific functional groups. Assemblies with polymers may also contribute to increased porosity. For example, AlMashat and collaborators ${ }^{[148]}$ reported a hydrogen sensor based on an assembly of polyaniline (PANI) adsorbed on the rGO surface, synthesized by in-situ polymerization of aniline selectively on rGO surface. The hybrid rGO/PANI displayed a $16.6 \%$ response to $1 \% \mathrm{H}_{2}$ gas, which was better compared to pure rGO and PANI (Figure 20 a-b). An assembly of rGO and 


\section{WILEY-VCH}

PANI has been used also to sense $\mathrm{NH}_{3} \cdot{ }^{[149]}$ Conductive polymers have also been used to assemble with GO gas-sensitive conductive hydrogels by Shi and co-workers. ${ }^{[150]}$ In particular, hydrogels of GO/polypyrrole (PPy), GO/PEDOT, and GO/PANI were fabricated by in-situ chemical polymerization of the corresponding monomers in aqueous dispersions of GO. The GO/PPy gel in particular, once lyophilized, exhibited a good sensitivity to $\mathrm{NH}_{3}$ gas. LbL assembly of GO with charged polyelectrolytes have also been used for gas sensing; for example, Zhang and co-workers ${ }^{[130]}$ developed a flexible, resistive-type humidity sensor based on the LbL assembly of GO and poly(diallyldimethylammonium chloride) (PDDA). After the alternate deposition of GO and PDDA for 5 consecutive cycles on interdigitated electrodes, the assembly was chemically reduced by $\mathrm{HBr}$ and used to sense moisture. The device exhibited a $37 \%$ response (from $0 \% \mathrm{RH}$ to $97 \% \mathrm{RH}$ ), excellent linearity and a long stability over time (Figure 20 c-e). Chemically modified GO/rGO decorated with small functional molecules has also been used for gas sensing. For instance, we showed recently that it is possible to greatly enhance the humidity sensing performances of $\mathrm{rGO}$ by functionalizing it with hydrophilic molecules, i.e. triethylene glycol chains. ${ }^{[151]}$ The resistive sensor was produced by depositing the functionalized material between two gold electrodes. The sensing performances to humidity of this sensor were compared to a rGO sensor and to a hydrophobic decane decorated rGO. The $\mathrm{rGO} /$ triethylene glycol hybrid exhibited the best sensing performances, with ultrafast ( $25 \mathrm{~ms})$ response and recovery, high sensitivity $(0.31 \% / \mathrm{RH})$, high stability and selectivity to humidity. The choice of functional units allows adjusting the sensitivity to specific gases, as Jelinek et $a l .{ }^{[93 c]}$ showed recently in their work, in which they combined in a given sensors array three different porous GO functionalized with different dangling units (phenyl-GO, ethanol-GO and dodecyl-GO). The different response to different vapours of the three materials allowed the sensor to discern between water, $\mathrm{NH}_{3}$, toluene, $\mathrm{EtOH}$, phenol and cyclohexane. 


\section{WILEY-VCH}

a

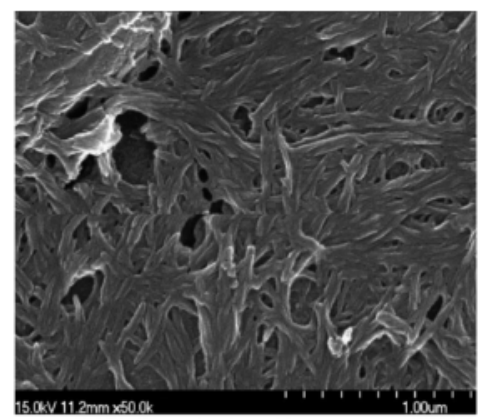

b

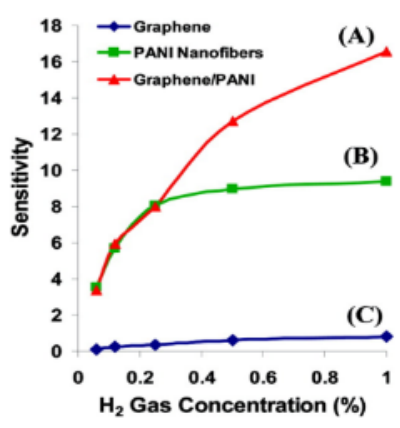

C
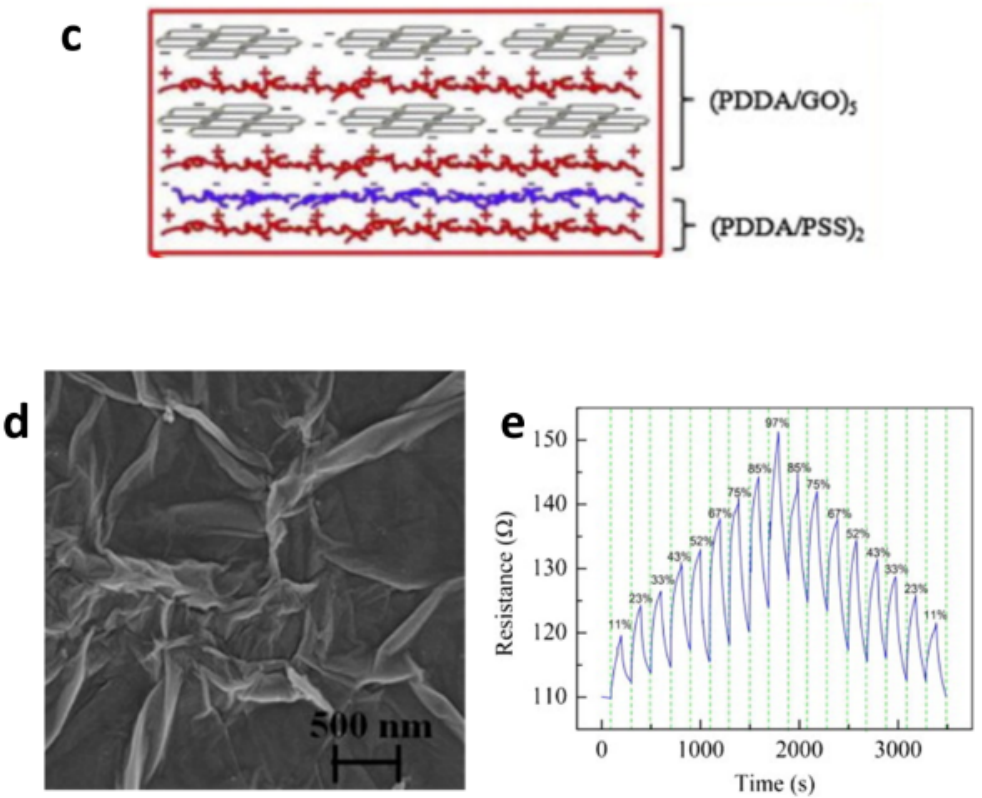

Figure 20: (a) SEM of rGO-PANI hybrid. b) Sensitivity of the rGO, rGO-PANI, and PANI sensors to different $\mathrm{H}_{2}$ concentrations. Adapted from Ref. ${ }^{[148]}$ Schematic (c) and SEM (d) of the PDDA/GO LbL assembly. (e) Resistance of the PDDA/GO sensor as a function of time at different RH \%. Adapted from Ref. ${ }^{[130]}$

Graphene-based hybrid materials have also been used for the sensing of metal ions in solution.

These sensors rely on different mechanisms of detections: electrical sensors such as resistive sensors, field-effect transistor (FET) based sensors and electrochemical sensors are the most diffused, however, fluorescence-based sensors have also been developed. For instance, flexible liquid-gated FET based on rGO have demonstrated their utility in real time monitoring of human sweat. ${ }^{[152]}$ In FET based sensors usually, the metal ions are adsorbed by the functional molecules or nanostructures that compose the hybrid and provoke a change in the Dirac point or carrier mobility of graphene metal, which is detected as a change in one of the FET characteristics such as the field-effect mobility, threshold voltage and $I_{\mathrm{on}} / I_{\mathrm{off}}$ ratio. In a typical example, Zhang et al. ${ }^{[153]}$ reported the sensing of $\mathrm{Hg}^{2+}$ with a FET based on mechanically exfoliated graphene functionalized with SAM of 1-octadecanethiol. The sensing of $\mathrm{Hg}^{2+}$ was detected as a shift in the Dirac point, and the detection limit was $10 \mathrm{ppm}$ of $\mathrm{Hg}^{2+}$. Furthermore, 


\section{WILEY-VCH}

they observed from AFM images of the sensor an increase in the height of $1.6 \mathrm{~nm}$ after the adsorption of $\mathrm{Hg}^{2+}$ by the thiol groups (Figure $21 \mathrm{a}-\mathrm{b}$ ). For the detection of heavy metal ions, hybrids of graphene with biomolecules are commonly used thanks to the high binding affinity and selectivity of the last to metal ions. Sudibya et al. ${ }^{[154]}$ presented a FET sensor based on a micro-patterned film of rGO functionalized via pyrene linkers with metal binding metallothionein type II protein for the selective recognition of $\mathrm{Hg}^{2+}$ and $\mathrm{Cd}^{2+}$. The high affinity of the protein for these two metals allowed the sensor to detect concentrations of $\mathrm{Hg}^{2+}$ and $\mathrm{Cd}^{2+}$ as low as 1nM. An et al. ${ }^{[140]}$ reported a flexible device based on CVD graphene functionalized non-covalently with an aptamer for detection of $\mathrm{Hg}^{2+}$. In particular, the aptamer was covalently bonded to a linker comprising a naphthalene unit attached via $\pi-\pi$ stacking to graphene. The graphene/aptamer hybrid was contacted with gold electrodes and used as liquidion gated FET sensor that was able to detect selectively $\mathrm{Hg}^{2+}$ ions with an extremely low detection limit of $10 \mathrm{pM}$ (Figure $21 \mathrm{c}-\mathrm{e}$ ). Graphene-aptamer complexes have also been used as fluorescent probes for the detection of metal ions, in which graphene acts by quenching the emission of a fluorophore or quantum dots. In other cases, GO itself may act as a fluorophore whose emission is quenched by the presence of the metal. One of the approaches is based on Förster resonance energy transfer (FRET) and it is useful for the quantitative determination of target molecules. For example, Li et al. ${ }^{[155]}$ fabricated a fluorescence probe $\mathrm{Hg}^{2+}$ ions based on a supramolecular assembly between GO and single-stranded DNA (ssDNA) aptamer. The $\mathrm{GO} / \mathrm{ssDNA}$ assembly is dispersed in solution and exhibits strong fluorescence emission of GO at $600 \mathrm{~nm}$; in presence of $\mathrm{Hg}^{2+}$ ions, the ssDNA folds to form double-stranded DNA complexed with the metal ions, which quench the emission of GO. An assembly of GO and a short ssDNA fragment has been used by Wen et al. ${ }^{[156]}$ to sense $\mathrm{Ag}^{+}$ions. In this case, a ssDNA with high affinity for $\mathrm{Ag}^{+}$ions was used. The DNA bears attached a fluorescein molecule and is non- 


\section{WILEY-VCH}

covalently adsorbed on GO, which quenches the fluorescence of fluorescein. In presence of $\mathrm{Ag}^{+}$the DNA folds to complex the metal ions and is released from the GO surface; in this way the fluorescence of fluorescein is restored. This sensor showed very high selectivity to $\mathrm{Ag}^{+}$ versus 12 different interfering metal ions.
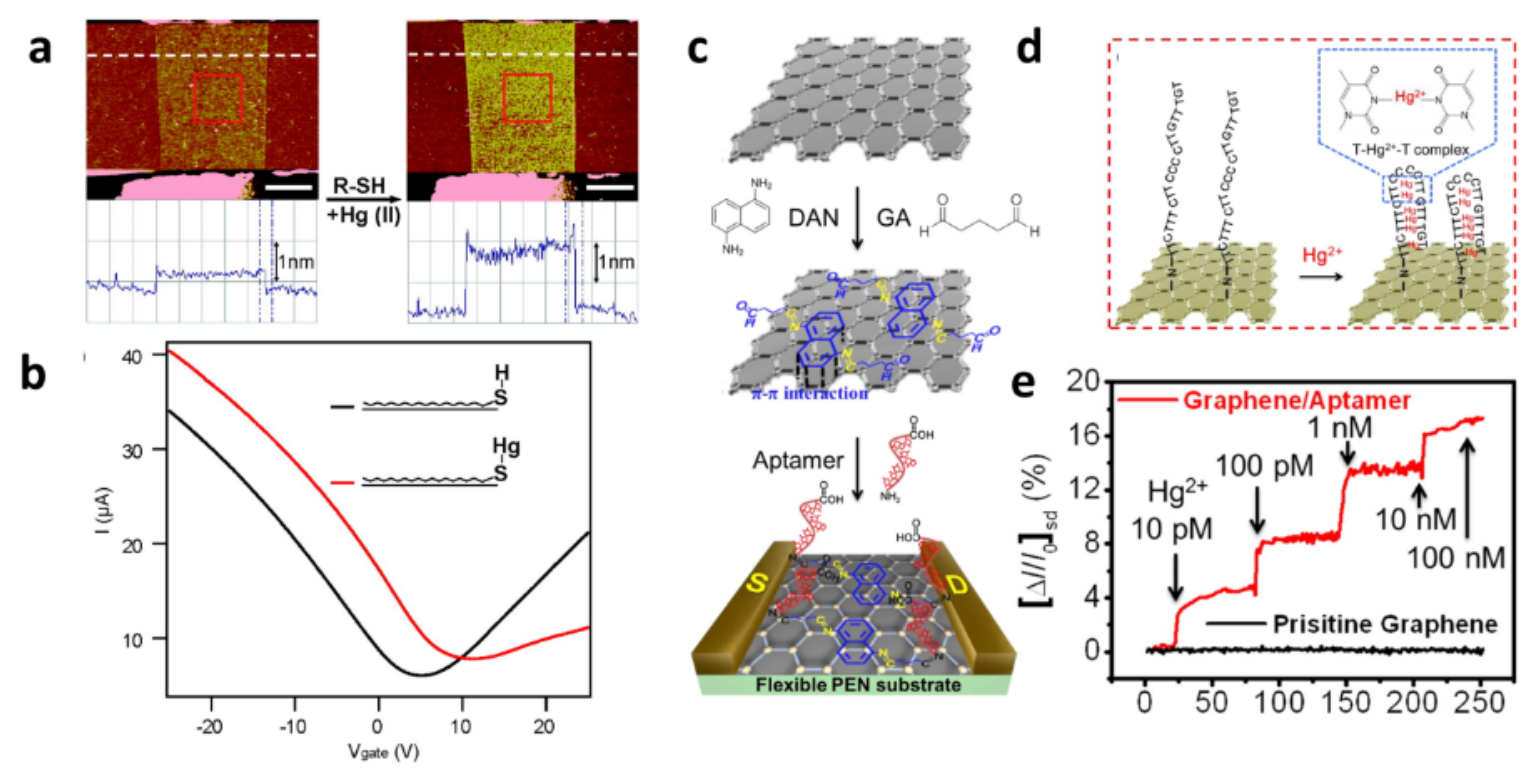

Figure 21: (a) AFM and height profiles of the mechanically exfoliated graphene functionalized with SAM of 1-octadecanethiol before (left) and after (right) the adsorption of $\mathrm{Hg}^{2+}$. (b) Transfer characteristic of the assembled FET device before (red) and after (black) $\mathrm{Hg}^{2+}$ adsorption. Adapted from Ref. ${ }^{[153]}$ (c) Schematic of the assembly steps of the CVD graphene/DNA aptamer device. (d) Schematic of the selective binding of the aptamer to $\mathrm{Hg}^{2+}$. (e) Real-time responses (\%) of the CVD graphene/aptamer device (red) and CVD graphene only (black) to various $\mathrm{Hg}^{2+}$ concentrations. Adapted from Ref. ${ }^{[140]}$

Fluorescence sensors have also been used to sense biomolecules, such as DNA. Dong and coworkers ${ }^{[27 c]}$ realized a GO/QDs hybrid capable of detecting specific DNA fragments. The QDs are functionalized with a molecular beacon $(\mathrm{MB})$ complementary to the target DNA. In absence of the analyte, the QDs are adsorbed on the GO surface by van der Waals interactions between the $\mathrm{MB}$ and $\mathrm{GO}$ and their fluorescence is quenched. In presence of the target DNA, the MB links it and straightens, bringing the QD far from the surface of GO and restoring its fluorescence. In addition to fluorescence sensors, biomolecules and organic molecules have 


\section{WILEY-VCH}

been detected by FET sensors and electrochemical sensors. For example, a FET sensor for target DNA fragments was produced by the non-covalent functionalization of rGO with a peptide nucleic acid. ${ }^{[157]}$ The highly specific interaction of the peptide nucleic acid with the complementary DNA strand caused a shift in the transfer characteristic curve of the FET; the sensor had a detection limit of $0.1 \mathrm{pM}$.

Electrochemical sensors are powerful tools for the selective determinations of molecules and ions in solution. In fact, the different molecules can be selectively detected by their different reduction and oxidation potentials. Graphene hybrid materials are used as free-standing electrodes or deposited on another electrode. The advantage of using graphene is its high surface area, that allows for efficient adsorption of the analyte, its low charge transfer resistance and relatively wide potential window. ${ }^{[27 a]}$ The sensitivity and selectivity of graphene can be enhanced by combining it with functional molecules or nanostructures. For instance, Guo et $a l .{ }^{[158]}$ reported a one-step, microwave-assisted fabrication of Pt NPs functionalized rGO for sensing $\mathrm{H}_{2} \mathrm{O}_{2}$ and the explosive trinitrotoluene (TNT). The hybrid material was deposited on a glassy carbon electrode (GCE) and used to sense various analytes by cyclic voltammetry. In particular, the modified electrode showed much higher current densities and sensitivities compared to the bare GCE and exhibited particular catalytic activity towards $\mathrm{H}_{2} \mathrm{O}_{2}$ and TNT which were detected in concentrations as low as $80 \mathrm{nM}$ and $1.3 \mu \mathrm{m}$ respectively. Luo et al. ${ }^{[159]}$ reported a rGO/Cu NPs modified GCE for the determination of glucose. The increased catalytic activity of this modified electrode allowed glucose detection in a concentration of $0.5 \mu \mathrm{M}$.

\subsubsection{Other kind of sensors}

Graphene hybrids have also been extensively investigated for pressure and strain sensors and temperature sensors. For example, Huang et al. ${ }^{[160]}$ developed a hybrid rGO/molecules highperforming pressure sensor. Tree different molecules of different stiffness were covalently 


\section{WILEY-VCH}

bonded to rGO by exploiting the epoxide ring opening reaction, then the material was deposited by spray-coating between two electrodes, forming a millefeuille-like structure in which the dangling molecules act as deformable pillar between the rGO layers. By applying a small pressure to the multilayer structure, the deformation of the pillars brings the rGO layer closer, favouring the electron tunnelling which ultimately yields a huge decrease in the electrical resistance of the device. Interestingly, the softer molecular pillar produced the highest sensitivity, which was as high as $0.82 \mathrm{kPa}^{-1}$, with a detection limit of just $7 \mathrm{~Pa}$. In another example, an ultrafast pressure sensor based on a hybrid multilayer structure of GO and graphene flakes sandwiched between two electrodes was produced. ${ }^{[161]}$ Chen et al. ${ }^{[38 \mathrm{c}]}$ built a strain sensor based on a stacked structure of a $\mathrm{rGO} / \mathrm{Ag}$ NWs hybrid deposited on a stretchable polymeric substrates. The deformation of the $\mathrm{rGO} / \mathrm{Ag}$ NWs network between $0.3 \%$ and $2 \%$, induced a change in the resistance of the strain sensor, with gauge factors as high as 4000 . A similar strain sensor based on a CVD graphene/Ag NWs hybrid deposited onto pre-stretched polyacrylate was produced by $\mathrm{Li}$ et al. ${ }^{[162]}$. This sensor was capable of detecting strains between $5 \%$ and $200 \%$, with response time below $1 \mathrm{~ms}$.

\subsection{Water purification}

The availability of potable water for the entire world population, which is constantly growing, has become a major concern in recent years. The scientific community is called to the challenge of developing new, more efficient technologies for the desalination of seawater and the removal of metallic and organic pollutants. Graphene, and in particular GO and rGO, have found promising applications as membranes for the desalination of water and as adsorbents for pollutants. One of the most employed technologies for water desalination is reverse osmosis and graphene may contribute to the construction of more efficient semipermeable membranes. Graphene has the unique property of being extremely thin, yet being impermeable to the 


\section{WILEY-VCH}

passage of small molecules and ions, ${ }^{[163]}$ nonetheless the creation of nanopores in the graphene structure may allow the selective permeability to water. ${ }^{[137,164]}$ While several methods to achieve the formation of nanopores of controlled size in graphene layers have already been developed, large continuous layers of graphene are still too expensive to be implemented into commercial products. ${ }^{[165]}$ A more affordable and achievable alternative is the use of multilayer stacks of GO/rGO flakes, which can form mechanically strong membranes semipermeable to water. ${ }^{[131,166]}$ In these, water molecules can pass through the narrow hydrophobic channels formed by the unoxidized regions of GO. ${ }^{200}$ The rejection of ions or bigger molecules takes place mainly for size exclusion since these cannot pass between the stacked GO flakes, yet anions can also be rejected by electrostatic interactions. ${ }^{[167]}$ However, over time GO membranes tend to lose the semipermeability as a result of the increase in the interlayer spacing between flakes. ${ }^{[168]}$ On the other hand, rGO membranes have higher stability but the narrower interlayer distance decreases also the permeability to water. The selective permeability to water and the stability of $\mathrm{GO} / \mathrm{rGO}$ membrane can be enhanced by combining GO with molecules or nanostructures that can increase the strength of the membrane by connecting together the different flakes and tune the size of the channels, thus permitting a higher flux of water and rejection of other molecules and ions. For example, $\mathrm{Hu}$ and $\mathrm{Mi}$ developed a membrane of stacked GO flakes, assembled by the LbL method in which the flakes were cross-linked by 1,3,5-benzenetricarbonyl trichloride (TMC) (Figure 22 a). ${ }^{[169]}$ The cross-linking of GO flakes not only increased the stability of the membrane against dissolution in water but also defined the spacing between the flakes. The membrane exhibited a water flux up to 10 times higher than that of common commercial nanofiltration membranes, and $95 \%$ rejection of Rhodamine-WT, however, the rejection of salt was low (Figure $22 \mathrm{~b}$-c). Endo et al. ${ }^{[136]}$ presented a selective water permeable membrane produced by spray-coating water dispersions of GO, few-layer 


\section{WILEY-VCH}

graphene flakes and deoxycholate onto a porous polysulfone support. The assembly was then cross-linked by $\mathrm{Ca}^{2+}$ ions. They showed that the insertion of deoxycholate in the structure allowed increasing the interlayer distances and the filtration performances and the rejection of $\mathrm{NaCl}$. This membrane was robust enough to withstand $120 \mathrm{~h}$ of cross-flow shear and exhibited a $\mathrm{NaCl}$ rejection of $85 \%$ and $96 \%$ for an anionic dye. The interlayer distance in the membrane can be engineered by introducing nanostructures such as CNTs or nanosheets. Recently Hiu et $a l .{ }^{[170]}$ fabricated a hybrid GO/laponite flakes layered membrane for the selective permeation. The strength of the membrane was assured by the strong electrostatic interaction between the negatively charged GO and the positive charged laponite nanosheet. The membrane showed an efficient rejection of basic fuchsin and brilliant yellow dyes. Gao et al. ${ }^{[171]}$ produced a $40 \mathrm{~nm}$ thick membrane composed by GO intercalated by single-walled carbon nanotubes (SWCNTs) that exhibited a water permeation of $660-720 \mathrm{~L} \mathrm{~m}^{-2} \mathrm{~h}^{-1} \mathrm{bar}^{-1}$ and a rejection $>97 \%$ for molecules greater than $1.8 \mathrm{~nm}$ (Figure 22 d-f). Similarly, Han et al. ${ }^{[40]}$ prepared a hybrid multilayer assembly of rGO and multiwalled carbon nanotubes (MWCNTs), in which MWCNTs act as spacers between the rGO flakes. The hybrid membrane showed a water flux up to $11.3 \mathrm{~L} \mathrm{~m}^{-2} \mathrm{~h}^{-1}$ bar $^{-1}$, almost double of that of $\mathrm{rGO}$ alone, high dye rejection $(>99 \%$ for Direct Yellow and $>96 \%$ Methyl Orange) and moderate salt rejection $(51.4 \%$ for $\mathrm{NaCl})$. Sun et $a l .{ }^{[172]}$ fabricated a layer stacked membrane of GO and ZnO NPs whose interlayer spacing could be finely adjusted by partial reduction of GO via UV-irradiation. The addition of ZnO NPs to GO made it possible to achieve a 5 fold increase of the permeability to water, while maintaining unaltered the rejection of organic dyes $(98.4 \%)$ and enhancing the antibacterial properties. 


\section{WILEY-VCH}
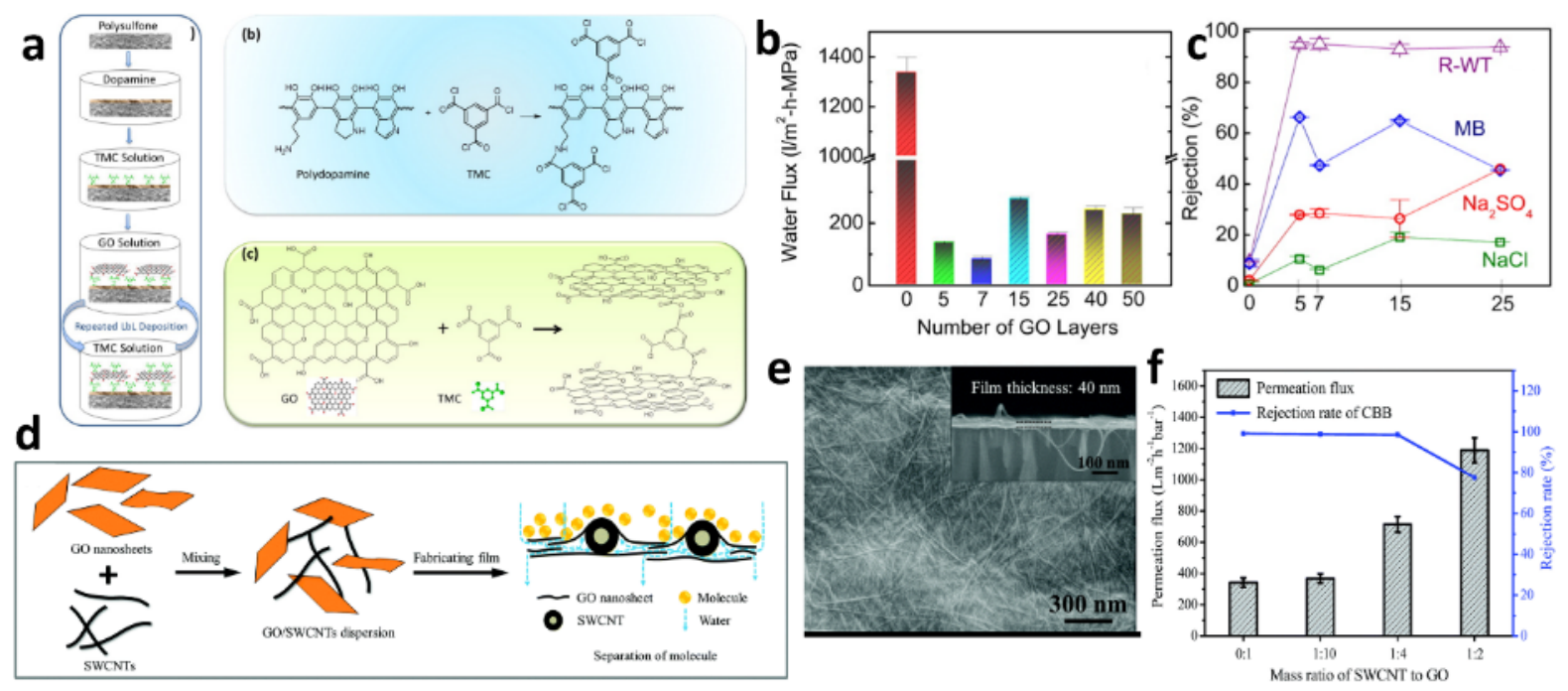

Figure 22: (a) Schematic representation of the LbL assembly between GO and TMC anchored at the polysulfone substrate by polydopamine. (b) Water permeation of the GO/TMC LbL structure and (c) Rejection in \% of the reported salts and dyes as a function of the number of layers. Adapted from Ref. ${ }^{[169]}$ (d) Schematic representation of the fabrication of the hybrid GO/SWCNTs and (b) SEM image of the hybrid (cross-section in the inset). (f) Permeability to water and rejection rate of Coomassie Brilliant Blue (CBB) as a function of the mass ration between SWCNTs and GO. Adapted from Ref. ${ }^{[171]}$

In addition to the insufficient availability of freshwater, water pollution by heavy metals and organic pollutants represents two other major problems brought into play by the population growth and the increasing use of the soil by humans. Water purification via removal of the pollutant can be accomplished by using adsorbents that sequester the pollutant via non-covalent interactions. An effective way to purify water is by adsorption of its contaminants on the surface of a material named absorber, which then can be easily removed. Adsorption depends on several parameters, such as $\mathrm{pH}$, temperature and concentration of the pollutant, and on the nature of the adsorbent. Graphene and graphene oxide have promising application as adsorbent mainly thanks to their large surface area and porosity. ${ }^{[29]}$ In particular, the lipophilicity of graphene (and $\mathrm{rGO}$ ) and its conjugated structures make them good adsorbents for aromatic and hydrophobic molecules, while the hydrophilicity and the numerous oxygen functional groups makes GO a good adsorbent for ionic species. In addition, the functional groups on GO can also act as bonding sites for further functionalization. The functionalization of these materials with 


\section{WILEY-VCH}

molecules or nanomaterials increases the surface area for adsorption, prevents the aggregation of the graphene/GO flakes that would result in a decrease of the adsorbing surface, and makes the adsorption more selective. ${ }^{[27 a, 29]}$ Nanomaterials, and nanoparticles in particular, have already been used for adsorbing pollutants from water because of their properties originating from their small size, such as large surface area and catalytic activity. ${ }^{[173]}$ However, bare nanoparticles may easily agglomerate due to a high surface free energy which results in a reduction of the active adsorption sites. The formation of hybrid assembly with graphene hinders the aggregation of both nanoparticles and graphene, yielding to a large increase in the adsorption capabilities. Moreover, if magnetic NPs are used, these can facilitate the separation of the adsorbent from water by means of a magnetic field. In 2010 Chandra et al. ${ }^{[21]}$ fabricated a hybrid $\mathrm{rGO} / \mathrm{Fe}_{3} \mathrm{O}_{4}$ magnetic NPs for the efficient removal of $\mathrm{As}(\mathrm{II})$ and $\mathrm{As}(\mathrm{V})(99 \%$ removal). The hybrid was synthesized by in situ simultaneous reduction of GO and Fe(II/III) precursors and exhibited uniformly distributed magnetite NPs on the rGO surface. The magnetic behaviour of the hybrid allowed for a simple separation from water (Figure 23 a-b). Following this work other papers based on assemblies of GO and rGO with iron-based magnetic nanoparticles have been presented, for increasing the adsorption efficiency of As(III/V) (e.g. by increasing the $\mathrm{pH}$ range of adsorption $)^{[174]}$ and for adsorbing other heavy metals such as $\mathrm{Pb}(\mathrm{II})^{[175]}, \mathrm{Cr}(\mathrm{VI})^{[34,175 b, 176]}, \mathrm{Hg}(\mathrm{II})^{[175 b]}$. In some cases, the magnetic nanoparticles have shown also efficient catalytic activity towards the reduction or oxidation of the metal ions to less toxic forms, for instance from $\mathrm{Cr}(\mathrm{VI})$ to $\mathrm{Cr}(\mathrm{III}) \cdot{ }^{[176]}$ As aforementioned, GO is a better adsorbent for heavy metal cations than $\mathrm{rGO}$, because of its negative charge and the presence of oxygen atoms that can coordinate the metals. Yang et al. ${ }^{[175 a]}$ decorated both GO and rGO with magnetic iron oxide NPs and demonstrated that the GO hybrid was a good adsorbent for $\mathrm{Pb}(\mathrm{II})$, but not for 1naphthol and 1-naphthylamine, while the rGO based hybrid behaved oppositely. In addition to 


\section{WILEY-VCH}

nanoparticles, GO and rGO functionalized with organic molecules and polymers have also found applications as adsorbents for metal ions and organic pollutants with increased adsorption capabilities and selectivity. Polymers with heterocyclic units such as polypyrrole (PPy) and polythiophene comprise heteroatoms that are capable to coordinate cations, making them good adsorbent for metal ions. Chandra and $\mathrm{Kim}^{[177]}$ prepared a highly porous structure of rGO functionalized non-covalently with PPy. This hybrid showed highly selective $\mathrm{Hg}(\mathrm{II})$ removal from water (Figure $23 \mathrm{c}-\mathrm{d}$ ). A hybrid of rGO and PPy was used by Li et al. ${ }^{[178]}$ for the removal of $\mathrm{Cr}(\mathrm{VI})$. In this case, the porosity was increased by templating the polymer over a $\mathrm{MnO}_{2}$ scaffold. Chitosan is another polymer with many coordinating groups that can form supramolecular assemblies with GO. Li et al. ${ }^{[179]}$ prepared an assembly of GO, chitosan and magnetic cyclodextrin containing a $\mathrm{Fe}_{3} \mathrm{O}_{4}$ core and used it for the adsorption of $\mathrm{Pb}(\mathrm{II})$. Zhang et $a l .{ }^{[180]}$ synthesized a biodegradable, highly porous hydrogel of chitosan and GO for the adsorption of heavy metal from aqueous solutions. Shahzad et al. ${ }^{[181]}$ synthesized a hybrid assembly of GO and EDTA-functionalized magnetic chitosan that exhibited maximum adsorption capacity $206.5 \mathrm{mg} \mathrm{g}^{-1}$ for $\mathrm{Pb}^{2+}$ and 207.2 for $\mathrm{Cu}^{2}$.
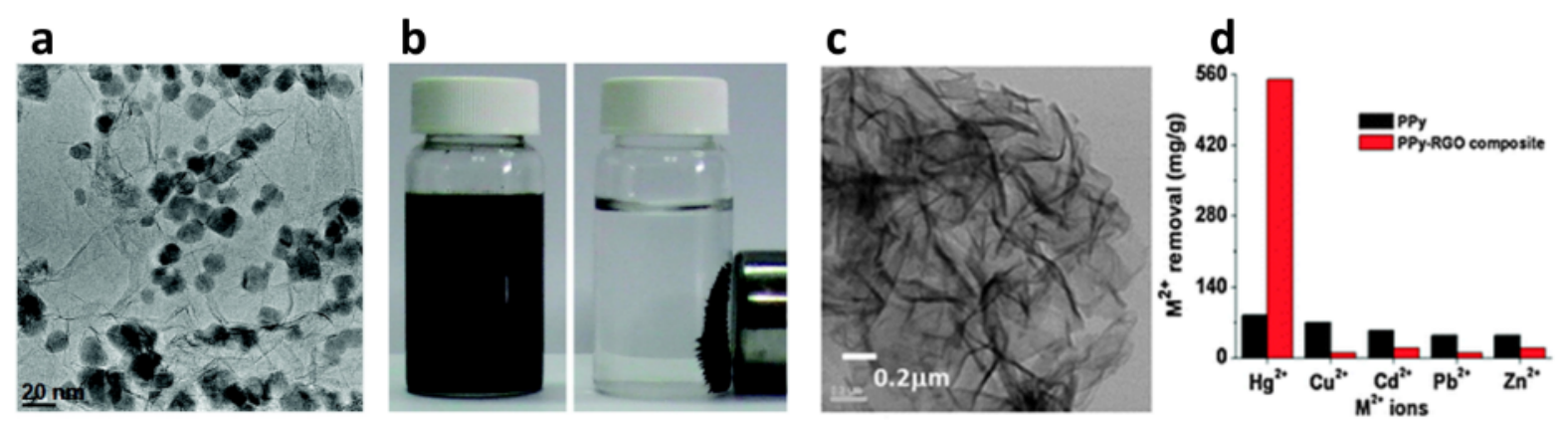

Figure 23: (a) TEM image of the $\mathrm{Fe}_{3} \mathrm{O}_{4} \mathrm{NPs} / \mathrm{rGO}$ hybrid and (b) magnetic separation of the adsorbent from solution. Adapted from Ref. ${ }^{[21]}$ (c) TEM image of the PPy/rGO hybrid. (d) Maximum adsorption capacity of various metal ions from aqueous solution using the PPy/rGO hybrid showing the high selectivity towards $\mathrm{Hg}(\mathrm{II})$. Adapted from Ref. ${ }^{[177]}$

Hybrid of GO and rGO have also been successfully employed as adsorbents for organic pollutants, e.g. dyes, polycyclic aromatic and halogenated organic compounds, oils, and 


\section{WILEY-VCH}

gasoline. Also in this case, magnetic hybrids have been largely investigated for the easier separation from water of the exhausted adsorbent. Different groups have synthesized hybrids of rGO and magnetic nanoparticles of $\mathrm{Fe}_{3} \mathrm{O}_{4}$, Fe or Ni via in-situ deposition methods and have used with them to absorb various aromatic dyes and gasoline. ${ }^{[29]}$ Fan et al. ${ }^{[182]}$ fabricated a magnetic chitosan GO hybrid and used it for the adsorption of methyl blue. The hybrid was synthesised by first coating $\mathrm{Fe}_{3} \mathrm{O}_{4}$ NPs chitosan, cross-linked by glutaraldehyde, and then grafted by covalent and non-covalent interactions to GO flakes. In this case, the adsorbing performances were greatly enhanced by the presence of chitosan, which can form ionic interactions between its amino groups and the negatively charged methyl blue. Instead of using dispersions of the hybrid adsorbent, which then need to be separated from water, another strategy consists in the use of monolithic sponge-like hybrids in form of foams or aerogels with high porosity that can be easily removed from water after the adsorption. These sponge-like hybrids are particularly useful to remove large quantities of spilt oils since when the oil is charged in the pores of the foam is retained there by hydrophobic effect. For instance, Dong et $a l .{ }^{[39 b]}$ fabricated a hybrid foam of CVD graphene/CNTs in which the CNTs grown over the graphene surface have the function of enhancing the hydrophobicity of the hybrid forming a nanostructured rough surface (Figure 24 a-c). The hybrid was formed by CVD deposition of graphene over a Ni foam template, followed by the CVD deposition of CNTs and dissolution of the Ni scaffold. The ultralight resulting foam exhibited a high adsorption capacity of different oils and organic solvents between 80 and 130 times the weight of the dry foam, and good recyclability (Figure 24 d). Shi and co-workers ${ }^{[183]}$ prepared a $\mathrm{rGO} / \mathrm{TiO}_{2}$ nanoneedles hybrid

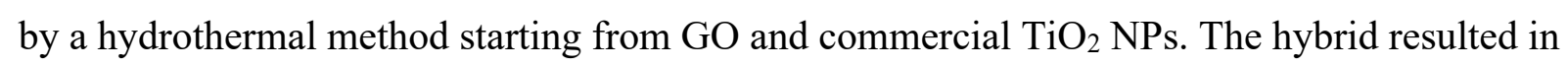
a highly porous structure with a good adsorption capacity of methylene blue $(83 \mathrm{mg} / \mathrm{g})$. Li et 


\section{WILEY-VCH}

$a l .{ }^{[184]}$ prepared a mesoporous hybrid of rGO coated by $\mathrm{Mg}(\mathrm{OH})_{2}$ via hydrothermal method and demonstrated its adsorption capabilities towards methylene blue.
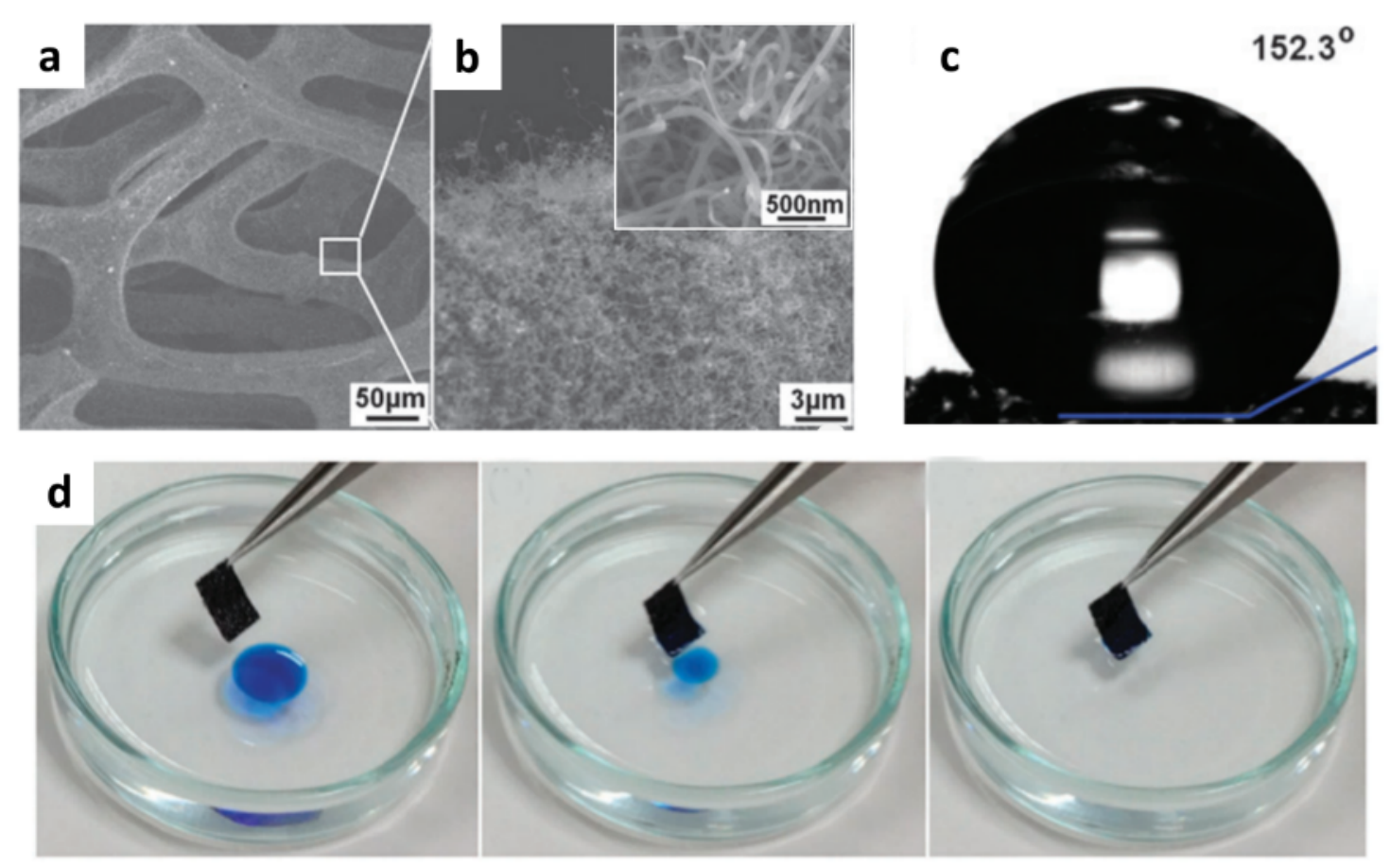

Figure 24: (a-b) SEM images of CVD graphene/CNT hybrid foam with different magnifications. (c) Water contact angle of the CVD graphene/CNT hybrid foam (the water contact Angle of the graphene foam without CNTs is $108.5^{\circ}$ ). (d) Photographs of the removal of a toluene droplet from water using the graphene/CNT hybrid foam. Adapted from Ref. ${ }^{[39 b]}$

\subsection{Catalysis, Photocatalysis and water splitting}

Hybrid assemblies based on graphene can be used as catalysts, to increase the rate of several reactions, and as photocatalysts. In particular, in photocatalysis the catalyst is usually a semiconductor which absorbs light, producing an exciton, in which one electron is excited from the valence band to the conduction band, that can then reduce or oxidize another molecule by donating it one electron or hole (Figure 25 a). The term photocatalysis can also include the use of a molecule, called photosensitizer, which absorbs light, forming an excited state that can either transfer the energy to another molecule, which, in turn, gets excited or react by oxidizing or reducing another molecule. Photocatalysis can be used to split water and produce oxygen 


\section{WILEY-VCH}

and hydrogen in the processes that are referred to as oxygen evolution reaction (OER) and hydrogen evolution reaction (HER). Furthermore, photocatalysis can also be used to degrade pollutant in water by formation of highly reactive radicals (such as $\bullet \mathrm{OH}$ and $\mathrm{O}_{2}^{-\bullet}$ ). An electrocatalyst instead is a catalyst used to increase the rate and reduce the overpotential of electrochemical reactions, in galvanic cells and electrolytic cells. Electrocatalysts are employed in fuel cells, for the oxygen reduction reaction (ORR) and hydrogen oxidation reaction (HOR). Pristine graphene is inert and has low catalytic activity; however, the chemical modification of graphene and the combination with nanostructures can greatly enhance its catalytic activity. ${ }^{[185]}$ Graphene can be combined with NPs or other nanostructures of materials that are commonly exploited for their catalytic activity, such as noble metals, transition metal oxides $\left(\mathrm{TiO}_{2}, \mathrm{Co}_{3} \mathrm{O}_{4}\right.$, $\mathrm{MnO}_{2}, \mathrm{Mn}_{3} \mathrm{O}_{4}$, etc..), transition metals chalcogenides (CdS, $\mathrm{ZnS}_{2}, \mathrm{Co}_{3} \mathrm{~S}_{4}, \mathrm{MoS}_{2}$, etc...) and nitrides, and greatly enhance their catalytic performances. ${ }^{[185-186]}$ Graphene can offer a large area where the catalytic nanostructures can be uniformly distributed, preventing their aggregation. Moreover, the electrical and mechanical properties of graphene confer to the hybrid electrical conductivity, with enhanced charge separation, increased mechanical strength, thermal and chemical stability. Graphene itself can become a good catalyst after the substitutional doping with nitrogen atoms, especially as electrocatalyst for ORR. ${ }^{[187]}$

The most investigated hybrid of graphene for catalytic applications comprises $\mathrm{TiO}_{2} \mathrm{NPs}$. $\mathrm{TiO}_{2}$ in fact is one of the prototypical materials used in photocatalysis for its superior photocatalytic properties, low cost, high availability, stability, and nontoxicity. Several synthetic techniques have been employed to prepare $\mathrm{TiO}_{2} /$ graphene hybrids, which include UVassisted photoreduction, ${ }^{[188]}$ sol-gel, ${ }^{[189]}$ hydro/solvothermal, ${ }^{[190]}$ and self-assembling methods. ${ }^{[191]}$ Most frequently, GO is used because of its low cost, easy processability and dispersibility in water. The same $\mathrm{TiO}_{2}$ nanoparticles can catalyse its photoreduction to rGO. ${ }^{[192]}$ 


\section{WILEY-VCH}

For instance, Zhang et al. ${ }^{[189]}$ prepared a $\mathrm{rGO} / \mathrm{TiO}_{2}$ nanocomposite for photocatalytic HER. The hybrid, containing just $5 \%$ of rGO, exhibited an evolution of $\mathrm{H}_{2}$ almost two times higher than commercial standard $\mathrm{TiO}_{2} \mathrm{P} 25$. The enhancement was primarily due to the conductivity of the rGO substrate, which collects the electrons and lowers the charge-recombination rate. Xiang et al. ${ }^{[190 \mathrm{a}]}$ prepared a $\mathrm{rGO} / \mathrm{TiO}_{2}$ nanosheets hybrid via a microwave-assisted hydrothermal method for the photocatalytic HER using methanol as sacrificial reagent (Figure $25 \mathrm{a}-\mathrm{b})$. The hybrid with $1 \%$ of $\mathrm{rGO}$ showed a $\mathrm{H}_{2}$ production rate of $736 \mu \mathrm{mol} \mathrm{h}^{-1} \mathrm{~g}^{-1}, 41$ times superior to the pure $\mathrm{TiO}_{2}$ nanosheets. Hybrids of $\mathrm{rGO}$ and $\mathrm{TiO}_{2}$ have also been extensively investigated for the photocatalytic degradation of organic pollutants. ${ }^{[190 \mathrm{~b}, 190 \mathrm{c}, 193]}$ The potential of $\mathrm{rGO} / \mathrm{TiO}_{2}$ hybrids for the degradation of organic pollutants was shown in 2009 by Zhang et $a l .{ }^{[190 \mathrm{~b}]}$, who presented a hybrid of $\mathrm{rGO}$ and commercial $\mathrm{TiO}_{2} \mathrm{P} 25 \mathrm{NPs}$ synthesized via hydrothermal method that could efficiently adsorb methylene blue from water and then photodegrading it under visible light irradiation. The efficiency of photocatalysis was 4.3 times higher than P25 alone. These performances were overtaken 3 years later by Lee et al. ${ }^{[190 \mathrm{c}]}$ who prepared crystalline $\mathrm{TiO}_{2} \mathrm{NPs}$ wrapped into rGO sheets. The material was prepared by one-step hydrothermal reduction of $\mathrm{GO}$ and phase change from amorphous $\mathrm{TiO}_{2}$ to crystalline (Figure $25 \mathrm{c})$. The band-gap of $\mathrm{TiO}_{2}$ in the hybrid was significantly reduced to $2.8 \mathrm{eV}$ and the efficiency of photocatalysis of the hybrid towards the degradation of methylene blue was 13 times higher than P25 NPs (Figure 25). 


\section{WILEY-VCH}
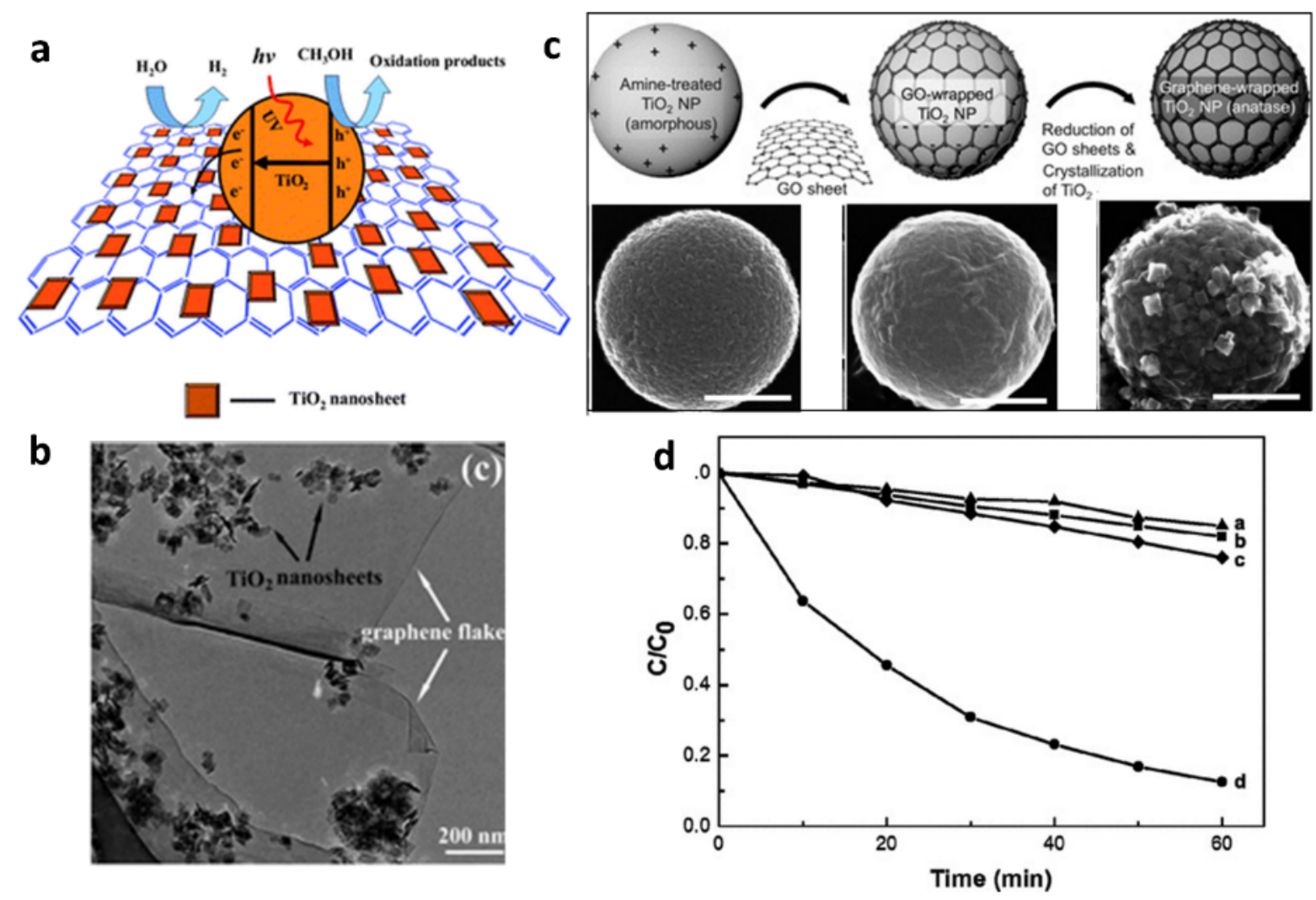

Figure 25: (a) Schematic illustration for the charge transfer and separation in the $\mathrm{rGO} / \mathrm{TiO}_{2}$ nanosheets hybrid under UV light irradiation; (b) TEM image of the hybrid. Adapted from Ref. ${ }^{\text {[190a] }}$ (c) Schematic illustration of synthesis steps for rGO-wrapped crystalline $\mathrm{TiO}_{2} \mathrm{NPs}$ and corresponding SEM images. (d) Photodegradation of methylene blue under visible light by (a) $\mathrm{P} 25$, (b) bare anatase $\mathrm{TiO}_{2} \mathrm{NPs}$, (c) $\mathrm{rGO} / \mathrm{TiO}_{2} \mathrm{NPs}$ (two-step hydrothermal), and (d) rGO/crystalline $\mathrm{TiO}_{2}$ NPs. Adapted from Ref. [190c]

Hybrids of graphene with noble metals, transition metals and metal oxides are largely employed for electrocatalysis. In particular, Pt is the most used catalyst in fuel cells; however, it suffers from various drawbacks, including low durability. ${ }^{[185]}$ The combination of Pt with graphene can greatly enhance performances and durability of Pt electrocatalysts. Yoo and co-workers ${ }^{[24 b]}$ found out that the deposition of Pt on rGO nanosheets produced nanoclusters of Pt below 0.5 nm. The nanostructuration of $\mathrm{Pt}$ modified its electronic structures producing a great enhancement in the electro-oxidation of methanol. Wan et al. ${ }^{[194]}$ developed in situ preparation of rGO decorated with hollow Pt nanocrystals. Also in this case, the synergic integration of Pt with rGO, and the nanostructuration provoked an enhancement in the electro-oxidation of 


\section{WILEY-VCH}

methanol. The nanostructuration can be obtained by using templates. Tiwari et al. ${ }^{[195]}$ showed that the deposition of Pt onto rGO could be templated by ssDNA. Depending on the Pt salt precursor used both Pt nanoclusters $(\sim 1 \mathrm{~nm})$ and Pt nanodendrites could be deposited (Figure 26 a). The synthesized $\mathrm{Pt} / \mathrm{ssDNA} / \mathrm{rGO}$ hybrids displayed very high catalytic activities towards the ORR, outdoing a Pt/C commercial catalyst. Furthermore, the hybrid catalyst was stable in a wide range of $\mathrm{pH}(1-13)$ and presented impressive durability and stability (95\% of performances after 10000 cycles) (Figure 26 b-c). Since Pt is highly expensive, alternative catalysts based on cheaper transition metals and metal oxides have been developed, and their combination with graphene showed promising enhancement of their performances. For instance, $\mathrm{Co}_{3} \mathrm{O}_{4}$ alone displays low catalytic activity towards ORR and OER. Dan et al. ${ }^{[196]}$ fabricated a hybrid of amine doped rGO and $\mathrm{Co}_{3} \mathrm{O}_{4}$ NPs (Figure 26 d). The synergic combination of the two materials produced a great enhancement in ORR and OER compared to the single component and to $\mathrm{Pt} / \mathrm{C}$ catalyst. Furthermore, compared to $\mathrm{Pt} / \mathrm{C}$, the hybrid catalyst showed higher durability and stability in alkaline media (Figure 26 e).
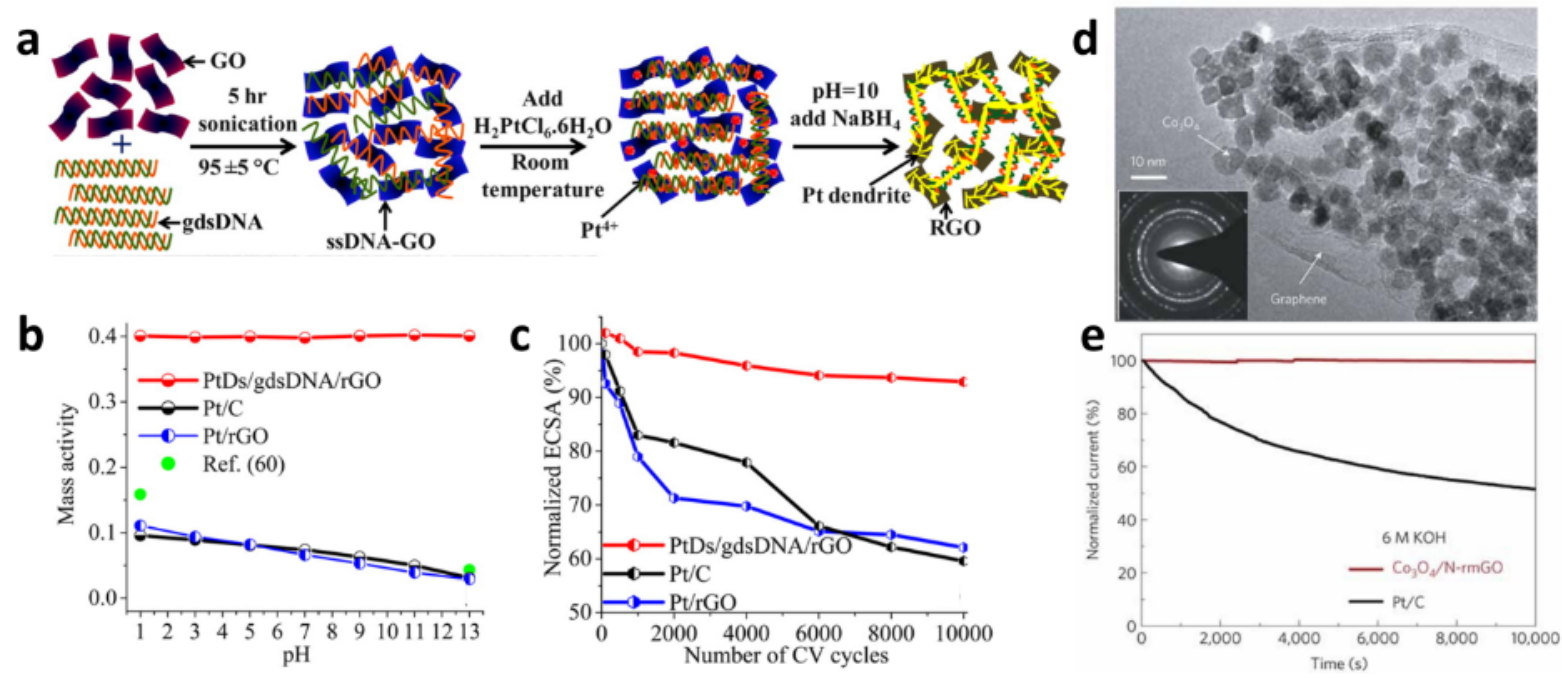

Figure 26: (a) Schematic of the synthesis of rGO/Pt Nanodendrites hybrid. (b) Mass activity of the catalysts reported in $\mathrm{O}_{2}$-saturated solutions of various $\mathrm{pH}$. (c) Electrochemically active surface area (EASA) loss for the catalysts reported, in $\mathrm{O}_{2}$-saturated $0.1 \mathrm{M} \mathrm{HClO}_{4}$ solution with a cyclic potential scan between 0.6 and $1.2 \mathrm{~V}$ at a scan rate of $50 \mathrm{mV} / \mathrm{s}$. Adapted from Ref. ${ }^{[195 \mathrm{a}]}$ (d) TEM image of the $\mathrm{N}$ doped $\mathrm{rGO} / \mathrm{Co}_{3} \mathrm{O}_{4}$ NPs hybrid. (e) Chronoamperometric responses of 


\section{WILEY-VCH}

$\mathrm{N}$ doped $\mathrm{rGO} / \mathrm{Co}_{3} \mathrm{O}_{4} \mathrm{NPs}$ hybrid and $\mathrm{Pt} / \mathrm{C}$ on carbon fibre paper electrodes kept at $0.70 \mathrm{~V}$ versus $\mathrm{RHE}$ in $\mathrm{O}_{2}$-saturated $6 \mathrm{M} \mathrm{KOH}$ electrolyte. Adapted from Ref. ${ }^{[196]}$

Hybrids of graphene with TMDs have also exhibited catalytic activity and showed promising application for substituting Pt in HER. For example, Li et al. ${ }^{[197]}$ reported a hybrid of rGO sheets decorated with $\mathrm{MoS}_{2}$ NPs, synthesized through a one-step solvothermal reaction in dimethylformamide, using $\left(\mathrm{NH}_{4}\right)_{2} \mathrm{MoS}_{4}$ as $\mathrm{MoS}_{2}$ precursor. The hybrid displayed excellent catalytic activity towards HER, being far superior to that of the single components and similar to that of platinum (Figure $27 \mathrm{a}-\mathrm{c}$ ). The high performance was attributed to the strong electronic coupling between the $\mathrm{MoS}_{2} \mathrm{NPs}$ and rGO. Zheng et al. ${ }^{[198]}$ presented a hybrid rGO decorated with $\mathrm{MoS}_{2}$ nanosheets, synthesized via solvent-evaporation-assisted intercalation of $\mathrm{MoS}_{2}$ precursor in graphite oxide, followed by the exfoliation and reduction. The formation of $\mathrm{MoS}_{2}$ nanosheets was templated by the narrow space existing between the graphite oxide layers. The hybrid exhibited excellent HER activity with small onset overpotential of $140 \mathrm{mV}$ (Figure 27 d-f). These performances were attributed to the nanostructuration of $\mathrm{MoS}_{2}$ with abundance of exposed active edge sites and to the excellent electrical conductivity of rGO. Van der Waals vertical heterostructures of $\mathrm{MoS}_{2}$ and graphene, produced by CVD, have also displayed excellent performances in photocatalytic HER. ${ }^{[199]}$ Different stacks were studied and the bilayer stack with graphene on top of $\mathrm{MoS}_{2}$ showed the highest photoresponse with large reaction current density and lowest charge-transfer resistance toward HER, with performances far superior to $\mathrm{Pt} / \mathrm{C}$ catalyst. Theoretical calculations revealed that $\mathrm{MoS}_{2}$ induced $\mathrm{p}$-type doping in graphene, facilitating the hydrogen adsorption on the graphene side, with $\Delta G_{\mathrm{H}}$ close to $0 \mathrm{eV}$. 


\section{WILEY-VCH}
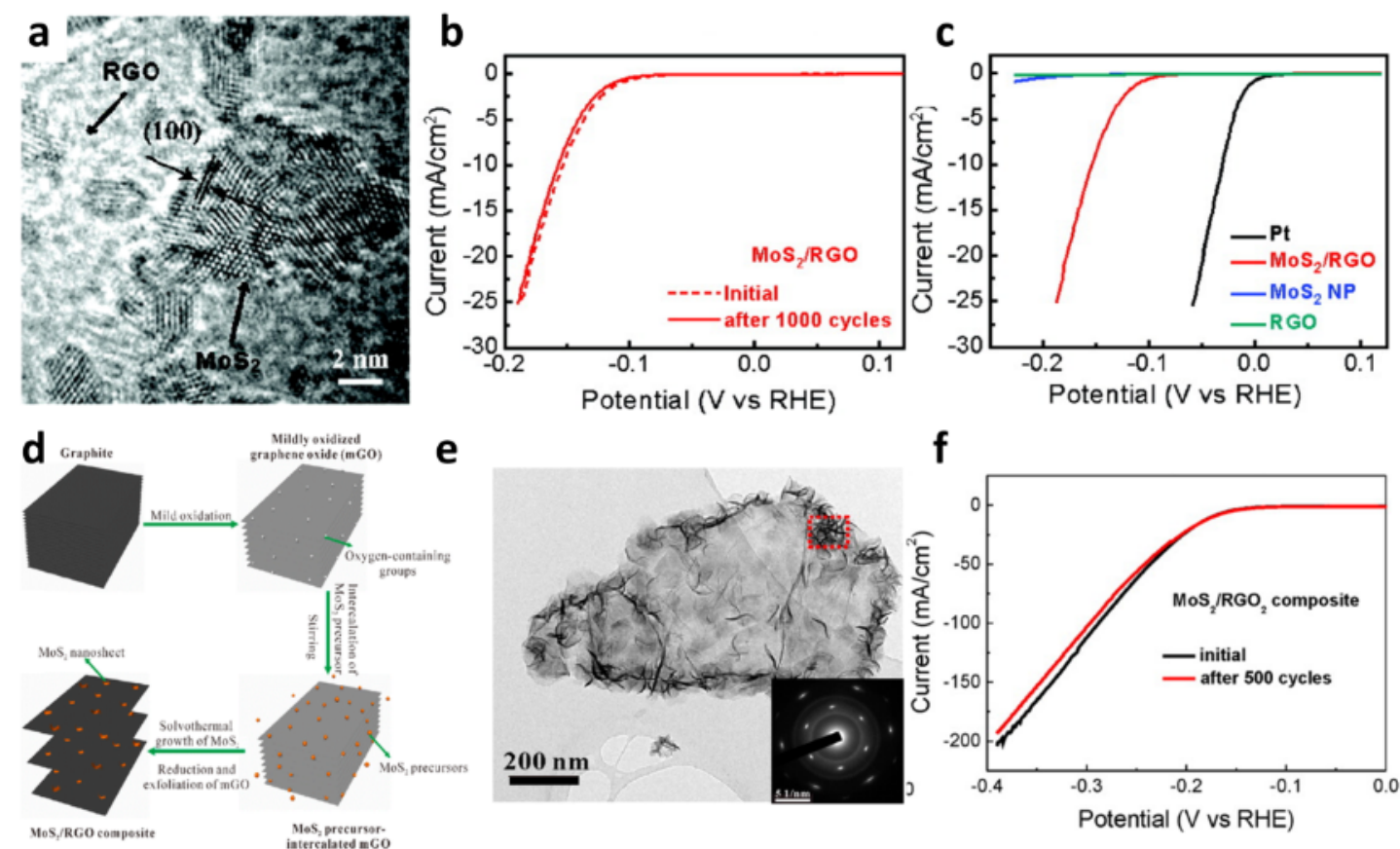

Figure 27: (a) TEM image of the $\mathrm{rGO} / \mathrm{MoS}_{2}$ NPs hybrid and (b) durability test of the hybrid catalyst after 1000 cycles from -0.3 to $+0.7 \mathrm{~V}$ at $100 \mathrm{mV} / \mathrm{s}$. (c) Polarization curves obtained with several catalysts as indicated recorded on glassy carbon electrodes with a catalyst loading of $0.28 \mathrm{mg} / \mathrm{cm}^{2}$. Adapted from Ref. ${ }^{[197]}$ (d) Schematic of the synthesis of $\mathrm{rGO} / \mathrm{MoS}_{2}$ nanosheets hybrid and (e) TEM image of the same. (f) Durability test of hybrid after 500 cycles from 0 to $+0.4 \mathrm{~V}$ at $100 \mathrm{mV} / \mathrm{s}$ in $0.5 \mathrm{M} \mathrm{H}_{2} \mathrm{SO}_{4}$ solution. Adapted from Ref. ${ }^{[198]}$

\subsection{Biomedical applications}

In addition to their capacity to sense biological molecules, graphene-based hybrids have demonstrated promising application in drug and gene delivery, bioimaging, and photothermal/photodynamic therapy. Nonetheless, the use of graphene for medical applications still raises many concerns in the scientific community and in the general public because of the potential material's toxicity. Many studies about the toxicity of graphene have been performed; still, notably different results have been reported, mainly because of the high diversity between the available graphene types, e.g. in size and chemical composition. ${ }^{\left[133 b,{ }^{200]}\right.}$ Furthermore, the functionalization of graphene surface can greatly modify its toxicity. Pristine graphene is highly hydrophobic; thus, it has found limited application in the biomedical field. GO and rGO have been more extensively studied because of the easier processability in water dispersions. In 


\section{WILEY-VCH}

particular, the GO and rGO properties of fluorescence quenching ability, surface-enhanced Raman scattering (SERS), facile non-covalent and covalent functionalization and amphiphilic behaviour make GO and rGO based hybrids interesting for biomedical applications. The functionalization of the surface of GO is highly desirable in order to increase its biocompatibility. Furthermore, the availability of multiple active sites in GO and rGO allows for the parallel functionalization with drugs, chromophores, nanoparticles, and specific cellular receptors, making it a perfect versatile platform for theranostic. The first example of functionalized GO for biomedical application dates back to 2008 in a paper of Sun and coworkers. ${ }^{[201]}$ The authors produced nanosheets of GO (NGO), covalently functionalized with PEG chains to increase its dispersibility in biological fluids such as serum. These nanosheets were photoluminescent in the visible and infrared regions, which is particularly useful for livecell imaging because of the little fluorescence background in the NIR range. To this end, NGO/PEG was covalently conjugated to a B-cells specific antibody (Rituxan) for the selective binding to B-cell lymphoma cells. The specific binding to B-cells was confirmed by illuminating the biological sample at $658 \mathrm{~nm}$ and recording its photoluminescence. Furthermore, the antibody marked NGO/PEG was used for loading the cancer drug doxorubicin via $\pi-\pi$ stacking and selectively killing the target cancer cells. The delivery via functionalized GO of potent aromatic drugs in the body has been especially investigated because their insolubility has often hampered their therapeutic use. In another example, Liu et al. ${ }^{[202]}$ prepared an amino-PEG decorated NGO, and attached to it via $\pi$ - $\pi$ stacking the aromatic camptothecin SN38 (Figure 28 a). They demonstrated the stability in biological liquids and high in vitro cell toxicity (Figure 28 b). More importantly, no toxicity was found supplying the cells with the same concentration of functionalized NGO without SN38 (Figure 28 c). Wang et al. . $^{[203]}$ showed that rGO nanoflakes functionalized with gold nanoclusters had higher efficacy in the 


\section{WILEY-VCH}

unloading of the drug doxorubicin in the cells, and the presence of the gold nanoclusters further inhibited the growth of the cells. In addition, the AuNPs/rGO retained IR fluorescence, which could be used for imaging of the cells. Other groups showed that the functionalization of $\mathrm{GO} / \mathrm{rGO}$ drug carriers with folic acid could greatly increase their selectivity towards the cancer cells, which are known to express many folate receptors. ${ }^{[204]}$ Functionalized rGO has showed also promising applications in biomedical applications; however, it is worth to note that the reduction method used has shown to influence its toxicity. In particular, GO reduced with hydrazine or ammonia has disclosed higher toxicity than GO reduced thermally or chemically with milder non-toxic molecules. ${ }^{[205]}$ The higher absorbance of rGO in the IR region compared to GO has been exploited for photothermal therapy. Yang et al. ${ }^{[206]}$ firstly demonstrated the use of functionalized rGO for the in vivo photothermal treatment of a tumour in a mouse. rGO was covalently functionalized with PEG chains and labelled with a fluorescent chromophore (Figure 28 d). By using fluorescent imaging they proved that, after administration to the mouse, the rGO/PEG was passively loaded preferentially in the cancer cells (Figure $\mathbf{2 8}$ e). Then they used low-power laser IR irradiation for the photothermal treatment of the tumour, achieving ultra-efficient tumour ablation. In comparison, the $\mathrm{rGO} / \mathrm{PEG}$ alone and the laser irradiation alone did not have any effect on the reduction of the tumour (Figure 28 f). Furthermore, histological and blood analysis did not show any side-effect of the rGO/PEG. In another example, PEG functionalized GO was decorated with $\mathrm{Fe}_{3} \mathrm{O}_{4}$ and $\mathrm{Au}$ NPs, which allowed for the imaging of the hybrid by using magnetic resonance and X-Ray. ${ }^{[31 \mathrm{a}]}$ The irradiation of the tumour in vitro provoked its destruction resulting from the combined effect of temperature and reduction of $\mathrm{GO}$, with release of $\mathrm{CO}_{2}$. Other research groups showed that the efficiency of photothermal therapy can be increased by loading a chemotherapeutic drug on the rGO carrier. ${ }^{[133 b]}$ 


\section{WILEY-VCH}
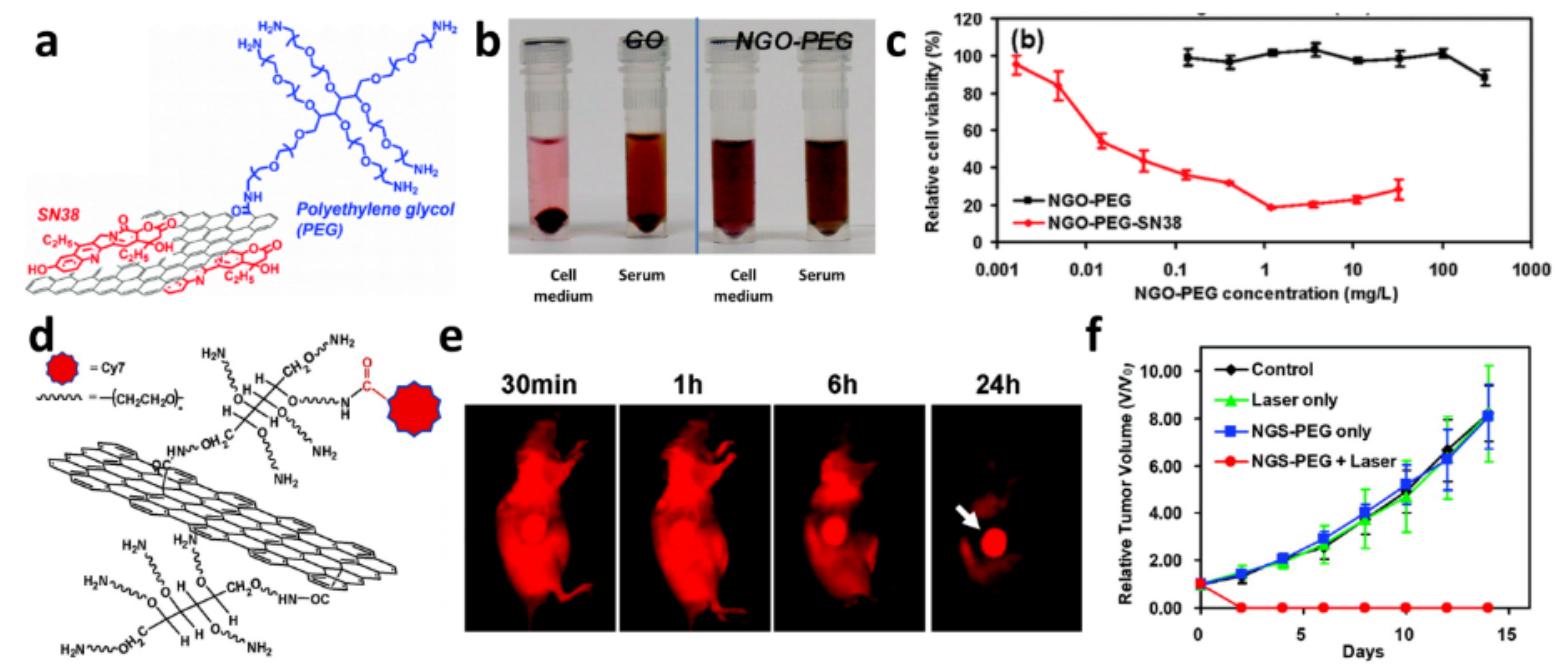

Figure 28: (a) Schematic draw of SN38 loaded NGO/NPEG. (b) Photos of GO and NGO/PEG in cell medium and serum recorded after centrifugation at $10000 \mathrm{~g}$ for $5 \mathrm{~min}$. (c) Relative cell viability data of HCT-116 cells after incubation with NGO-PEG with (red) and without (black) SN38 loading. Adapted from Ref. ${ }^{[202]}$ (d) Schematic of NGO/PEG labeled by Cy7 chromophore. (e) In vivo fluorescence images of U87MG tumour-bearing mice at different times post-injection of NGO/PEG-Cy7. (f) Tumour growth curves of different groups of untreated mice and mice after treatment with laser alone, NGO/PEG alone and NGO/PEG + laser. Adapted from Ref. [206]

Hybrids of GO and rGO have also been employed for photodynamic therapy; in these cases, $\mathrm{GO} / \mathrm{rGO}$ is functionalized with a photosensitizer, and this upon irradiation gets excited and induces the formation of reactive oxygen species (ROS), which are highly toxic for the cells. $\mathrm{GO} / \mathrm{rGO}$ functionalized with molecules such as porphyrin, methylene blue and chlorin have been tested. For example, Rong et al. ${ }^{[207]}$ attached a modified chlorin photosensitizer (Photochlor ${ }^{\circledR}$ ) onto PEG-functionalized GO via supramolecular $\pi-\pi$ stacking. The in vivo distribution and delivery were tracked by fluorescence imaging and showed a much higher loading of the GO hybrid into the cancer cells compared to the photosensitizer alone, which increased its photodynamic therapy efficacy. Functionalized GO and rGO have been also investigated for protein and gene delivery. For example, Feng and co-workers ${ }^{[208]}$ have reported a non-covalent supramolecular assembly of GO and PEI, which were bonded by electrostatic interaction. The same was used to bind negatively charged plasmid DNA to the positively 


\section{WILEY-VCH}

charged PEI (Figure 29 a). PEI also had the function of facilitating the release of the DNA into the cell. However, while PEI alone exhibited cytotoxicity, no toxicity was observed for the assembly GO/PEI (Figure 29 b). As aforementioned, GO has been largely employed for biomedical applications because of its easy functionalization and hydrophilicity. The downside of this higher reactivity of GO compared to pristine graphene is that GO may react with proteins and cellular structures within the body, thus leading to cellular damage and inflammation. Furthermore, GO may not be stable in the reducing environment of the living cells. For this reason, Lucarelli et al. ${ }^{[209]}$ presented recently the first example of functionalized pristine graphene as a multifunctional platform for imaging, chemotherapy and photothermal therapy. Liquid-phase exfoliated graphene nanosheets were covalently functionalized via diazonium salt chemistry with three different functional groups, which in turn were covalently attached to indocyanine green (ICG), PEG-folic acid and doxorubicin in a multi-step reaction (Figure 29 c). ICG allowed for the in vitro fluorescence imaging of the hybrid platform, while folic acid terminated PEG provided dispersibility in water and selective targeting of the cancer cells, and doxorubicin was used as a chemotherapeutic drug. This hybrid showed in vitro high selective loading in the cancer cells and good cell killing ability under laser irradiation by combination of photothermal and chemotherapy properties ( Figure 29 d). Furthermore, in vivo toxicological studies on mice of the non-functionalized graphene nanosheets showed no inflammatory response. Graphene based hybrids may contribute not only to the fight against cancer, but also to vanquishing viral diseases, such as Covid-19, and preventing future pandemics. ${ }^{[210]}$ For instance, graphene may be functionalized with ad-hoc receptors, such as sulphated polysaccharides and sulfonic acids able to inactivate viruses or to block their replication mechanism by binding irreversibly to them. ${ }^{[211]}$ In addition, coatings based on graphene functionalized with antiviral agents could be used to produce antiviral surfaces and items. 


\section{WILEY-VCH}
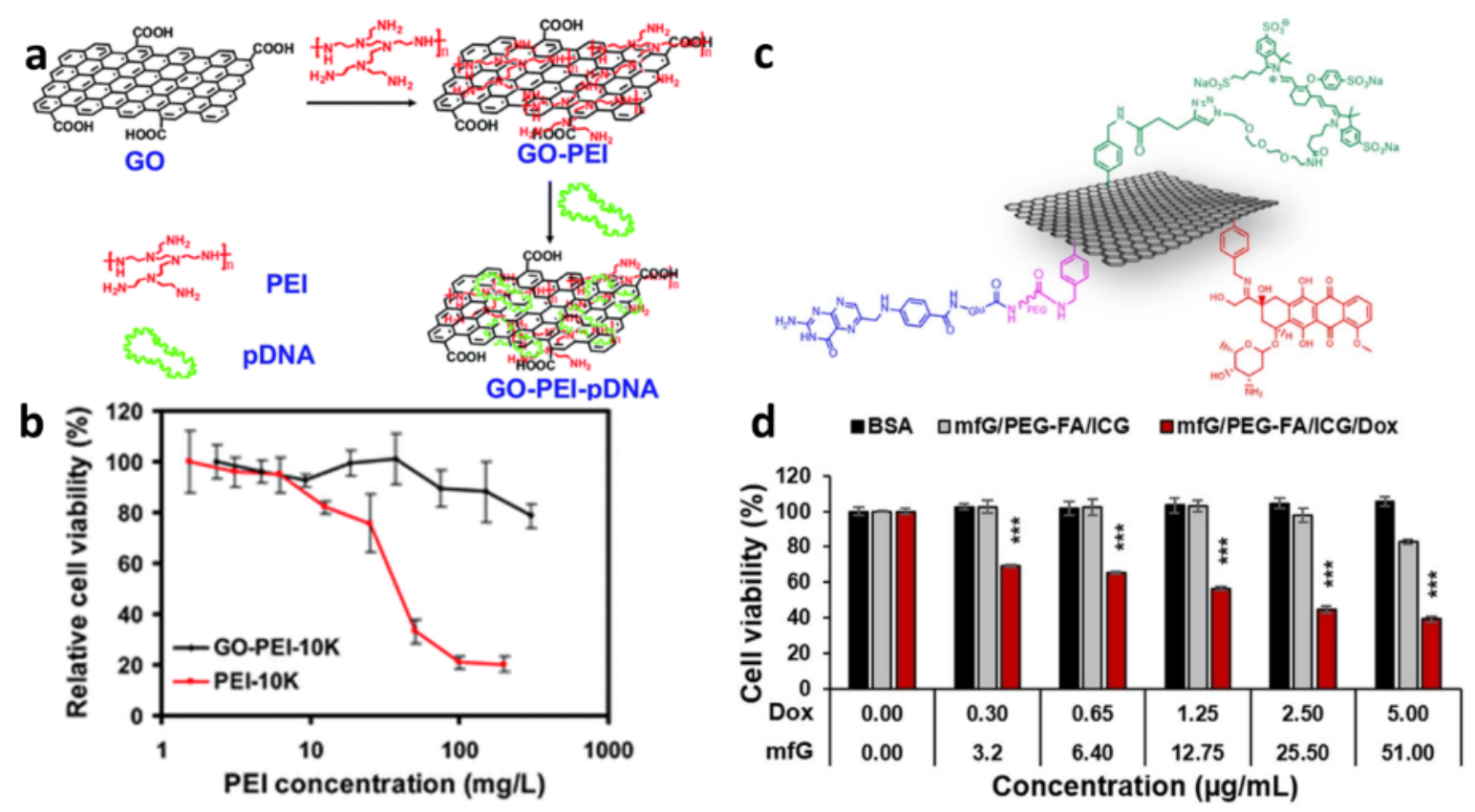

Figure 29: (a) Schematic representation of the assembling of the hybrid GO/PEI/pDNA via electrostatic interactions. (b) Relative cell viability data of HeLa cells incubated with different concentration of the GO/PEI and PEI alone (PEI molecular weight $10 \mathrm{KDa}$ ). Adapted from Ref. [208] (c) Schematic of the multifunctional graphene platform functionalized with IGC, PEGfolic acid and doxorubicin (mfG/PEG-FA/ICG/Dox). (d) Relative cell viability data of HeLa cells treated with bovine serum albumin (BSA), mfG/PEG-FA/ICG and $\mathrm{mfG} / \mathrm{PEG}-$ FA/ICG/Dox at different concentrations for $48 \mathrm{~h}$. Adapted from Ref. [209]

\subsection{Energy storage: supercapacitors and batteries}

The growth and development of renewable electricity sources, electrical cars and portable electronic devices are strongly dependent on the development of efficient energy storage systems such as lithium ions batteries and supercapacitors. Supercapacitors and batteries are complementary devices: the former possess high power density, but low energy density, while the latter possess high energy density and low power density. In both kinds of devices, research endeavour is primarily focussed towards the enhancement of the cyclability and durability in combination with the increase in the energy density and power density, which translate into devices that deliver more current, store more energy and have faster recharge times. The search for new electrode materials for Li-ion batteries and supercapacitors has found in graphene an excellent candidate, because of its electrical conductivity, ultrahigh surface area, chemical 


\section{WILEY-VCH}

stability and broad electrochemical window, mechanical strength joined with deformability and flexibility. The potential of graphene has emerged especially when this has been conjugated with electrochemically active nanomaterials.

Graphene-based hybrid materials are being investigated as anodes for lithium-ion batteries (LIB) and sodium-ion batteries (NIB). In particular, in sodium-ion batteries, the research for new anode materials is fundamental for the development of this technologies, since the traditional graphite based anodes employed for Li-ion batteries are completely inefficient in storing $\mathrm{Na}$ ions. ${ }^{[212]}$ Among these hybrid structures, graphene has the roles of increasing the electrical conductivity, accommodate the volume change of the electrode due to the Li-ions storage, stabilize and enhance the interface electrode/electrolyte, and improve the kinetic rates of the reaction with Li ions. ${ }^{[186]}$

One of the first examples of graphene-based hybrid for LIB anode was published in 2008 by Yoo and co-workers. ${ }^{[134 a]}$ The group prepared a paper-like electrode of $\mathrm{rGO}$ and CNTs or $\mathrm{C}_{60}$ fullerenes, produced by mixing GO flakes with hydrophilic oxidized CNTs and $\mathrm{C}_{60}$, followed by reduction with hydrazine and filtration. The integration of CNTs and $\mathrm{C}_{60}$ within the rGO structure produced an increase in the specific capacity up to $730 \mathrm{mAh} \mathrm{g}^{-1}$ and $784 \mathrm{mAh} \mathrm{g}^{-1}$, respectively, compared to the $540 \mathrm{mAh} \mathrm{g}^{-1}$ observed in neat $\mathrm{rGO} . \mathrm{SnO}_{2}$ has been indicated as a possible alternative to graphite as anode in LIB, with a theoretical capacity of $782 \mathrm{mAh} \mathrm{g}^{-1}$, being more than double to that of graphite. However, the main drawback of $\mathrm{SnO}_{2}$ is a great change in volume during the charge/discharge cycles which causes mechanical failures in the electrode, thus shortening the battery life. For this reason, Paek et al. ${ }^{[134 b]}$ fabricated a nanoporous hybrid of rGO flakes and $\mathrm{SnO}_{2} \mathrm{NPs}$ as anode for LIB, in which the porous nature of the hybrid and the flexibility or rGO accommodated the volume change of the $\mathrm{SnO}_{2} \mathrm{NPs}$ without breaking (Figure 30 a). The hybrid exhibited a specific capacity of $810 \mathrm{mAh} \mathrm{g}^{-1}$, and 


\section{WILEY-VCH}

a cycling performance drastically enhanced in comparison with that of bare $\mathrm{SnO}_{2} \mathrm{NPs}_{\text {(Figure }}$ 30 b). Later Zhou et al. ${ }^{[134 \mathrm{c}]}$ prepared a hybrid anode of $\mathrm{SnO}_{2}$ nanocrystals and $\mathrm{N}$-doped $\mathrm{rGO}$, by homogenizing $\mathrm{GO}$ and $\mathrm{SnO}_{2}$ nanocrystals in water solution, followed by freeze-drying and reduction with hydrazine vapours. The hybrid exhibited a huge specific capacity of $1352 \mathrm{mAh}$ $\mathrm{g}^{-1}$ and excellent retention of the performances after 500 cycles (Figure $30 \mathrm{c}-\mathrm{d}$ ). Hybrids of graphene and $\mathrm{TiO}_{2}$ have also been investigated as anodes for LIB. Wang et al. ${ }^{[213]}$ prepared a $\mathrm{rGO} / \mathrm{TiO}_{2}$ nanocrystals hybrid as anode for LIB. The material was prepared by in situ surfactant-assisted deposition of the $\mathrm{TiO}_{2}$ nanocrystals onto $\mathrm{rGO}$ flakes dispersed in water. The hybrid exhibited a specific capacity more than double compared to that of bare $\mathrm{TiO}_{2}$ nanocrystals, mainly due to its higher conductivity. $\mathrm{Mn}_{3} \mathrm{O}_{4}$ is a low-cost and abundant oxide, which however possesses little conductivity to be employed as anode in LIB. Wang et al. ${ }^{[214]}$ developed a low cost and environmental friendly anode for LIB based on a hybrid rGO/ $\mathrm{Mn}_{3} \mathrm{O}_{4}$ NPs. The material was synthesized by in situ one-pot deposition of the $\mathrm{Mn}_{3} \mathrm{O}_{4}$ NPs onto the surface of GO flakes and simultaneous reduction of GO to rGO under hydrothermal conditions. The hybrid exhibited a high specific capacity up to $\sim 900 \mathrm{mAh} \mathrm{g}^{-1}$, with good cycling stability. Silicon is another promising anode material for LIB because it has a large specific capacity, however, its great change in volume upon $\mathrm{Li}^{+}$insertion shortens the life of the batteries for the formation of fractures in the electrode. Graphene can accommodate the Si nanostructures deformation and protect them from the electrolyte. Lee et al. ${ }^{[215]}$ reported a simple method to produce a nanocomposite paper of $\mathrm{rGO}$ and $\mathrm{Si}$ NPs obtained by mixing the two components in water phase and filtering the dispersion. The hybrid presented a specific capacity of $2200 \mathrm{mAh}$ $\mathrm{g}^{-1}$ which decreased to $1500 \mathrm{mAh} \mathrm{g}^{-1}$ after 200 cycles. Luo et al. ${ }^{[216]}$ prepared submicrometersized capsules made of Si NPs wrapped by crumpled rGO shells, that were prepared by nebulization of an aerosol of GO and Si NPs dispersion, followed by drying of the microdroplets 


\section{WILEY-VCH}

in a tube furnace and formation of the final structure upon water evaporation (Figure 30 e-f).

The folds in the crumpled rGO coating can accommodate the volume expansion of Si NPs upon lithiation without fracture. This hybrid structure exhibited greatly improved performances compared to the bare Si NPs in terms of cycling stability and specific capacity (Figure $30 \mathrm{~g}$ ). Wang et al. presented a hierarchical multilevel architecture of Si NWs encapsulated with CVD graphene and in turn intercalated between rGO sheets. ${ }^{[37 \mathrm{c}]}$ Within the structure, the CVD graphene coating prevents the direct exposure of encapsulated silicon to the electrolyte enabling the structural and interfacial stabilization of silicon nanowires, while the conductive rGO sheets accommodate the volume change of embedded Si NW@G and maintain the structural and electrical integrity of the assembly. This hybrid electrode exhibited a specific capacity of 1600 $\mathrm{mAh} \mathrm{g}^{-1}$, high rate capability and $80 \%$ capacity retention after 100 cycles.
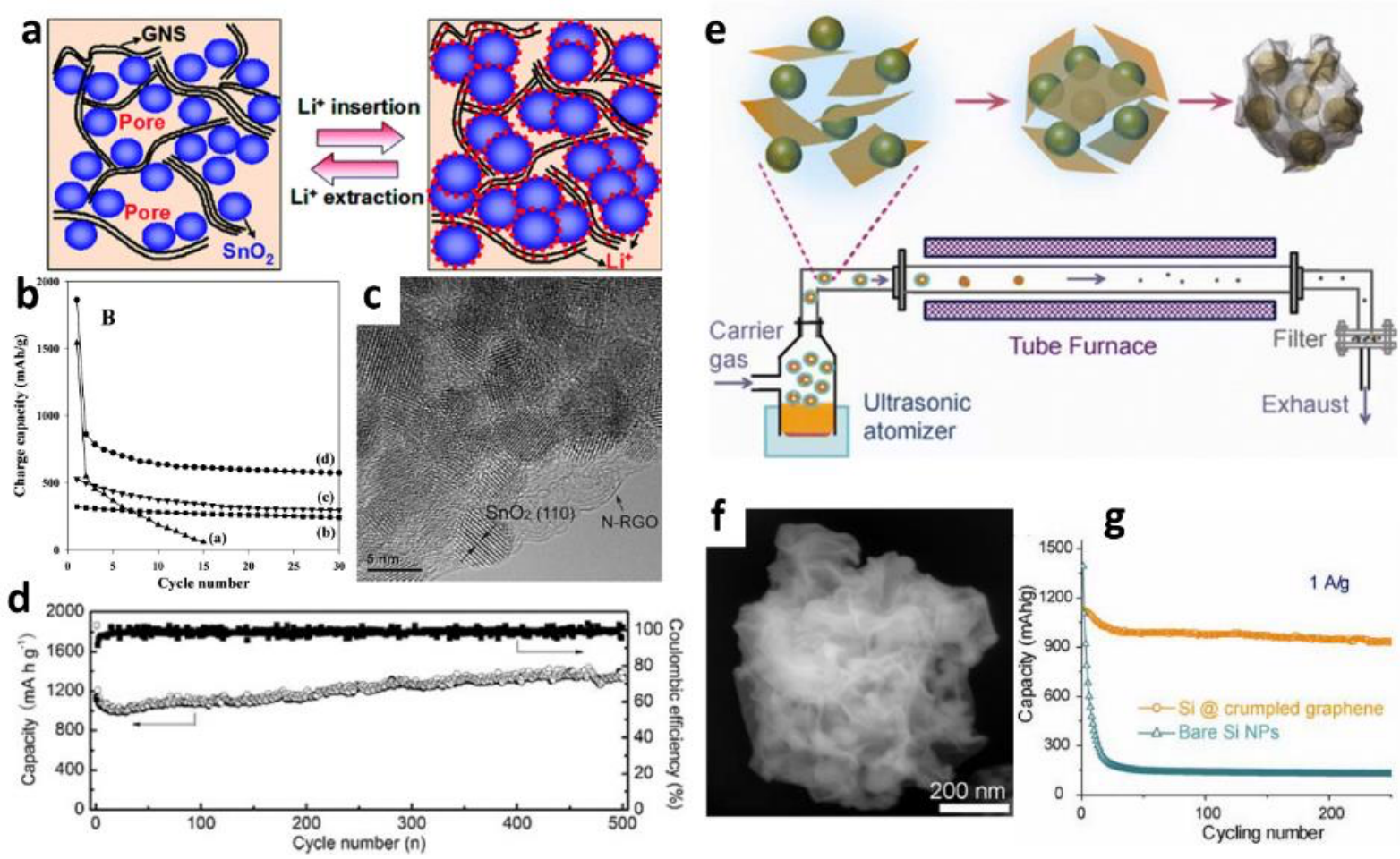

Figure 30: (a) Schematic of the structure $\mathrm{rGO} / \mathrm{SnO}_{2} \mathrm{NPs}$. (b) Cycling performances for (a) bare $\mathrm{SnO}_{2} \mathrm{NPs}$, (b) graphite, (c) $\mathrm{rGO}$, and (d) $\mathrm{rGO} / \mathrm{SnO}_{2}$. Adapted from Ref. ${ }^{[134 b]}$ (c) TEM image of $\mathrm{N}$-doped $\mathrm{rGO} / \mathrm{SnO}_{2}$ nanocrystals. (d) Cycling performance and Coulombic efficiency of the $\mathrm{N}-\mathrm{rGO} / \mathrm{SnO}_{2} \mathrm{NC}$ electrode. Adapted from Ref. ${ }^{[134 \mathrm{c}]}$. (e) Schematic of the synthesis of submicrometer-sized capsules made of Si NPs wrapped by crumpled rGO shells and (f) SEM 


\section{WILEY-VCH}

image of one of these capsules. (g) Cycling performances of the Si NPs/rGO capsules and bare Si NPs. Adapted from Ref. ${ }^{[216]}$

Sodium-ion batteries are being developed as cheaper and safer alternatives to Li-ion batteries. Since graphite cannot be used as anode in these batteries, hybrid architectures based on graphene seem the most promising choice. In $2013 \mathrm{Yu}$ et al. ${ }^{[217]}$ deposited crystalline $\mathrm{Sb}_{2} \mathrm{~S}_{3}$ NPs onto rGO flakes and employed the material as anode for NIB. The hybrid material presented a specific capacity of $730 \mathrm{mAh} \mathrm{g}^{-1}$, excellent rate capability and $97.2 \%$ capacitance retention after 50 cycles, compared to a $50 \%$ capacity retention of bare $\mathrm{Sb}_{2} \mathrm{~S}_{3}$. Sun et al. ${ }^{\left[{ }^{[56} \mathrm{b}\right]}$ fabricated a hybrid anode made out of few-layers phosphorene flakes sandwiched between graphene flakes, both obtained by ultrasonic-assisted liquid-phase exfoliation. In fact, phosphorene can react with sodium and lithium to form $\mathrm{Na}_{3} \mathrm{P}$ and $\mathrm{Li}_{3} \mathrm{P}$. The hybrid had a specific capacity of $2440 \mathrm{mAh} \mathrm{g}^{-1}$ (calculated on the phosphorene weight), and showed a $83 \%$ capacity retention after 100 cycles. In this case, graphene principally determined an enhancement of the electrical conductivity and mechanical properties, elastically supporting the volume changes of phosphorene. Recently, Liu et al. ${ }^{[218]}$ conceived a highly porous hierarchical flexible architecture as anode for NIB based on the structure of pillars and floors employed in modern buildings. The hybrid structure was composed by rGO layers spaced by carbon nanofibers (CNFs) interpenetrating them, all coated by $\mathrm{MoS}_{2}$ nanoflakes, for storing the $\mathrm{Na}$ ions. The structure was composed by firstly mixing CNFs, GO and poly(amic acid) (PAA) as binder, followed by directional freeze-drying and annealing under argon. Within this porous ordered architecture $\mathrm{MoS}_{2}$ nanoflakes were grown in situ. The hybrid anode demonstrated a specific capacity of $598 \mathrm{mAh} \mathrm{g}^{-1}$, long-term cycling stability up to 1000 cycles, and high rate capability up to $10 \mathrm{~A} \mathrm{~g}^{-1}$ (Figure 31). 


\section{WILEY-VCH}
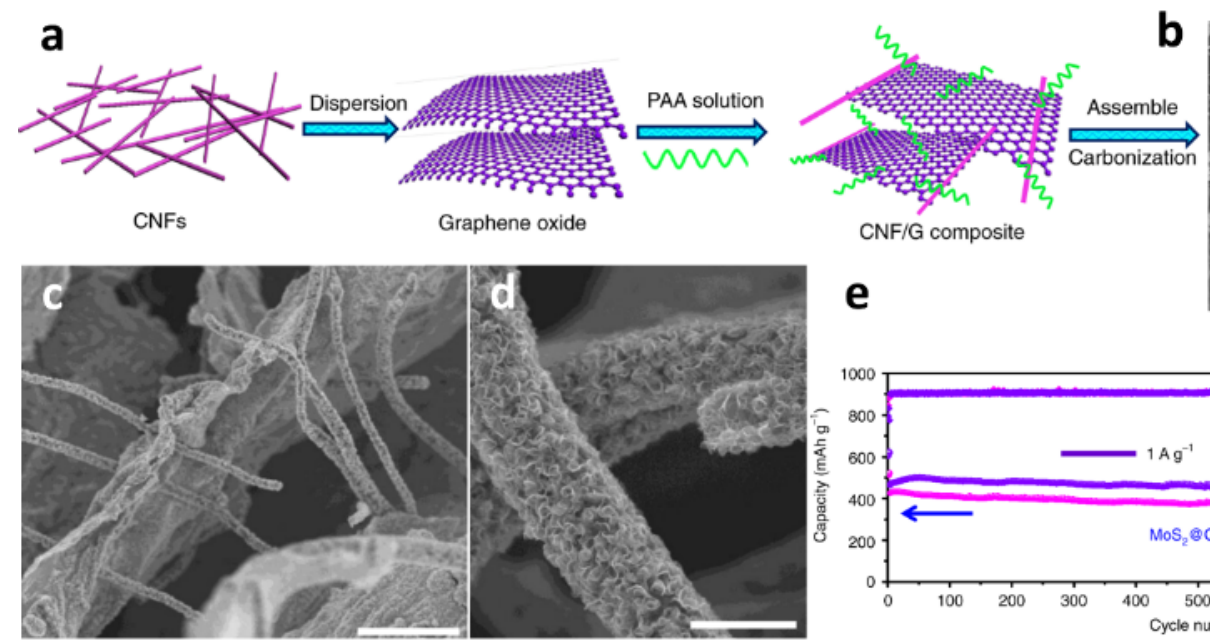

e
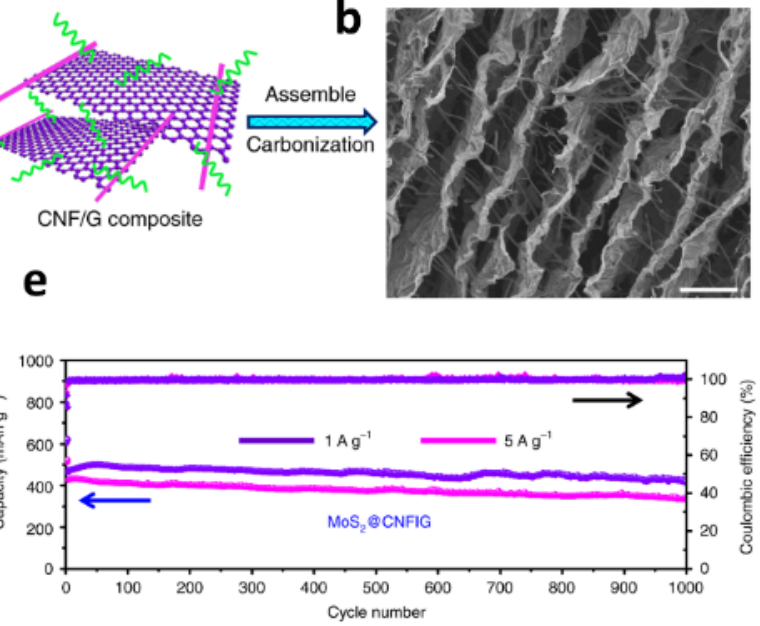

Figure 31: (a) Schematic of the synthesis of the hierarchical architecture CNFs/rGO and (b) SEM image of the structure after freeze-drying and thermal annealing. (c) and (d) SEM image of the CNFs/rGO/MoS 2 structure after the in-situ deposition of $\mathrm{MoS}_{2}$. (d) Specific capacity and coulombic efficiency as a function of the number of cycles for the $\mathrm{CNFs} / \mathrm{rGO} / \mathrm{MoS}_{2}$ anode. Adapted from Ref. ${ }^{[218]}$

Supercapacitors are devices capable of delivering high power densities and possessing short recharge and discharge times and much longer cycle life compared to batteries. In general, supercapacitors are based on two types of capacitance. One is electrostatic double-layer capacitance (EDLC), which is related to the formation of a thin region of charge separation at the interface of electrode and electrolyte. The other capacitance, also called pseudocapacitance, is related to the adsorption of ions on the electrode surface and to Faradic redox reactions between the electrode and the electrolyte ions. In EDLCs the first process is predominant, and usually, the electrodes used are carbon electrodes with large surface area, which do not participate in redox reactions with the electrolyte. Conversely, in pseudocapacitor, the Faradic redox processes prevail. In this case, electrochemically active electrode materials, such as metal oxides and conductive polymers are used. The two types of capacitance are present in hybrid and asymmetric supercapacitors. Hybrids of graphene with electrochemically active materials are promising electrodes for supercapacitors especially by virtue of their large surface area and high electrical conductivity, which permits fast electron transfer processes, and thus fast 


\section{WILEY-VCH}

charging and discharging times. ${ }^{[186]}$ Several metal oxides, nitrides, hydroxides and chalcogenides have been combined in hybrid with graphene, to build supercapacitors with superior performances. ${ }^{[186,219]}$ For instance, in 2010 Yan and co-workers ${ }^{[220]}$ presented a hybrid electrode composed by rGO decorated with $\mathrm{MnO}_{2}$ nanocrystals, synthesized by a one-step in situ deposition under microwave irradiation. The hybrid showed Faradaic pseudocapacitance, with a specific capacitance of $310 \mathrm{~F} \mathrm{~g}^{-1}$ at $2 \mathrm{mV} \mathrm{s}^{-1}$, and high capacitance retention at faster charge/discharge rates (Figure 32 a-b). A rGO/ $\mathrm{MnO}_{2}$ nanoplates hybrid electrode was presented by Peng et al. ${ }^{[221]}$, who prepared a symmetrical in-plane flexible supercapacitor on a PET film. The material was prepared by mixing in solution $\mathrm{rGO}$ and $\mathrm{MnO}_{2}$ nanoplates, which adhered to rGO via electrostatic interactions, and then by filtration of hybrid (Figure $32 \mathrm{c}-\mathrm{d}$ ). The film supercapacitor, with gel electrolyte, exhibited a high specific capacitance of $267 \mathrm{~F} \mathrm{~g}$ ${ }^{1}$ at current density of $0.2 \mathrm{~A} \mathrm{~g}^{-1}$ and capacitance retention of $92 \%$ after 7000 operating cycles (Figure 32 e). Xie et al. ${ }^{[222]}$ presented a N-doped rGO decorated with $\mathrm{MoS}_{2}$ nanoflowers, synthesized via a hydrothermal method. The material was then mixed with $10 \%$ Carbon black and deposited on a Ni foam electrode. The electrochemical characterization in $1 \mathrm{M} \mathrm{KOH}$ solution indicated a specific capacity up to $245 \mathrm{~F} \mathrm{~g} \mathrm{~g}^{-1}$ at $0.25 \mathrm{~A} \mathrm{~g}^{-1}$ and $91.3 \%$ capacitance retention after 1000 cycles at $2 \mathrm{Ag}^{-1}$. 


\section{WILEY-VCH}
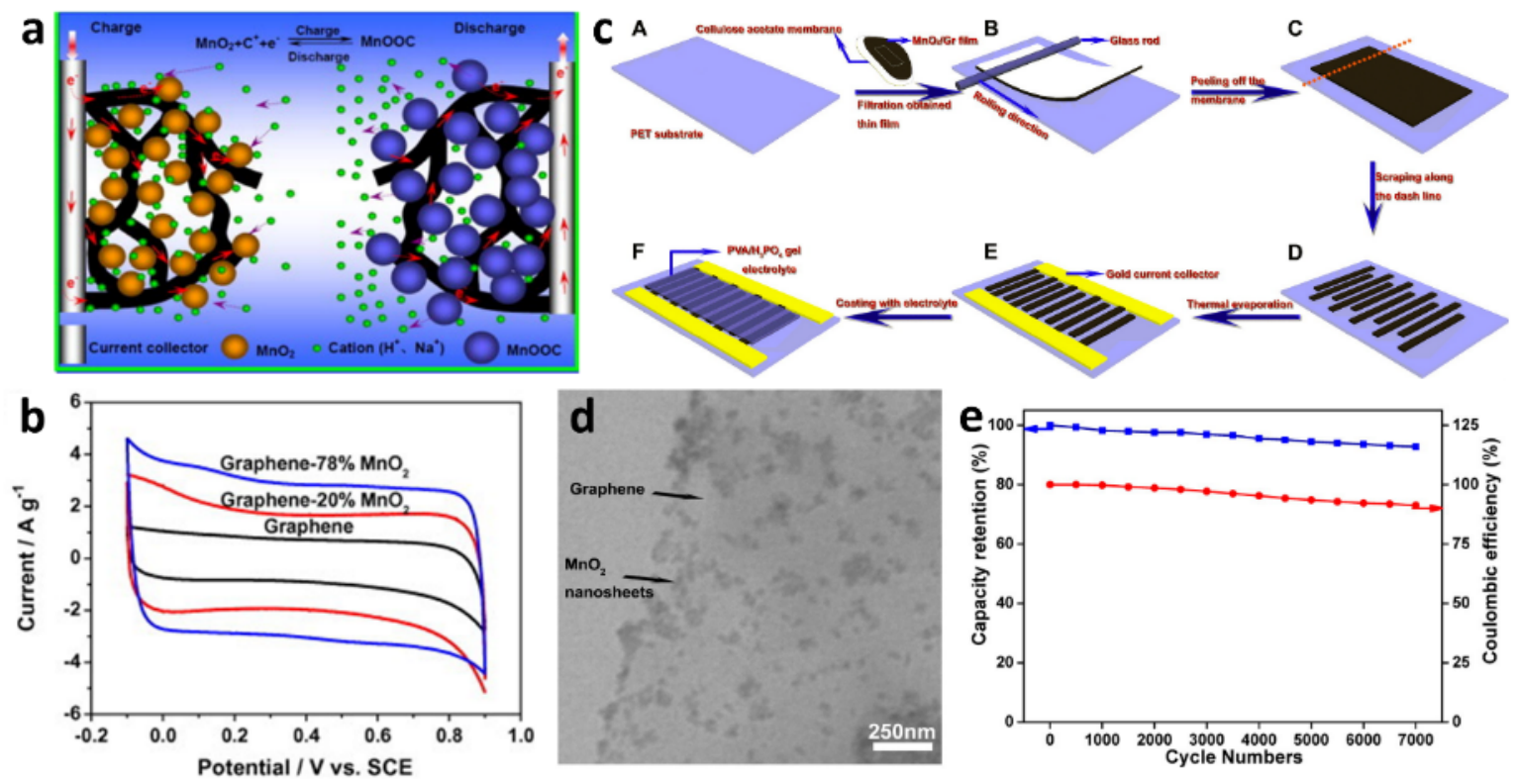

Figure 32: (a) Schematic illustration of the $\mathrm{rGO} / \mathrm{MnO}_{2}$ pseudocapacitor and Faradic redox reaction between $\mathrm{MnO}_{2}$ and metallic cations. (b) $\mathrm{CV}$ curves of rGO alone and $\mathrm{rGO} / \mathrm{MnO}_{2}$ composites at $10 \mathrm{mV} / \mathrm{s}$. Adapted from Ref. ${ }^{[220]}$ (c) Schematic of the fabrication steps of the flexible in-plane supercapacitor based on a $\mathrm{rGO} / \mathrm{MnO} 2$ hybrid. (d) TEM image of the $\mathrm{rGO} / \mathrm{MnO}_{2}$ hybrid. (e) Capacitance retention (blue) and Coulombic efficiency (red) of the in-plane supercapacitor over 7000 charge/discharge cycles. Adapted from Ref. ${ }^{221]}$

In order to increase the porosity of the electrode, thus increasing the available surface area and specific capacitance, Zhang and co-workers ${ }^{[142]}$ prepared a hybrid electrode based on rGO decorated with $\mathrm{Ni}_{3} \mathrm{~S}_{2}$ NPs and Bacillus subtilis bacteria, prepared by one-pot in situ deposition and simultaneous reduction of GO (Figure 33). After having deposited the hybrid on a Ni foam by electrospraying the electrode was tested in a $2 \mathrm{M} \mathrm{KOH}$ aqueous solution. Thanks to the high microporosity between the $\mathrm{RGO} / \mathrm{Ni}_{3} \mathrm{~S}_{2} \mathrm{NPs}$ conferred by the bacteria, the electrode exhibited a record specific pseudo-capacitance of $1424 \mathrm{~F} \mathrm{~g}^{-1}$ at a current density of $0.75 \mathrm{~A} \mathrm{~g}^{-1}$, with $67.5 \%$ of the capacitance retained at $15 \mathrm{~A} \mathrm{~g} \mathrm{~g}^{-1}$ and high cyclability $(89.6 \%$ capacitance retention after 3000 cycles at $15 \mathrm{~A} \mathrm{~g}^{-1}$ ) (Figure $33 \mathrm{c}$ ). Among the various strategies to increase the available surface area of the electrode, films of stacked graphene flakes intercalated by CNTs have been used. ${ }^{[36 a, 36 \mathrm{~d}]}$ CNTs create voids between the graphene layers and contribute to increasing the conductivity of the electrode. For instance, Xue et al. ${ }^{[36 \mathrm{~d}]}$ assembled an asymmetric flexible 


\section{WILEY-VCH}

solid-state supercapacitor in which the positive electrode was composed by alternated layers of rGO and $\mathrm{Ni}(\mathrm{OH})_{2}$ nanoplates and the negative electrode by rGO layers intercalated by CNTs (Figure 33 d-e). The two free-standing flexible electrodes were then sandwiched between PET films and separated by a gel electrolyte (Figure $33 \mathrm{f}$ ). The positive electrode displayed a specific capacitance of $573 \mathrm{~F} \mathrm{~g} \mathrm{~g}^{-1}$ and the assembled supercapacitor a volumetric capacitance of $58.5 \mathrm{~F} \mathrm{~cm}^{-3}$, with high cycling stability. Jung et al. ${ }^{[36 \mathrm{a}]}$ assembled a hybrid flexible electrode composed by rGO chemically bonded to CNTs via amide bond formation. The electrode exhibited a volumetric capacitance of $165 \mathrm{~F} \mathrm{~cm}^{-3}$, which was higher than that of the hybrid formed by physical mixing (Figure $33 \mathrm{~g}$ ).
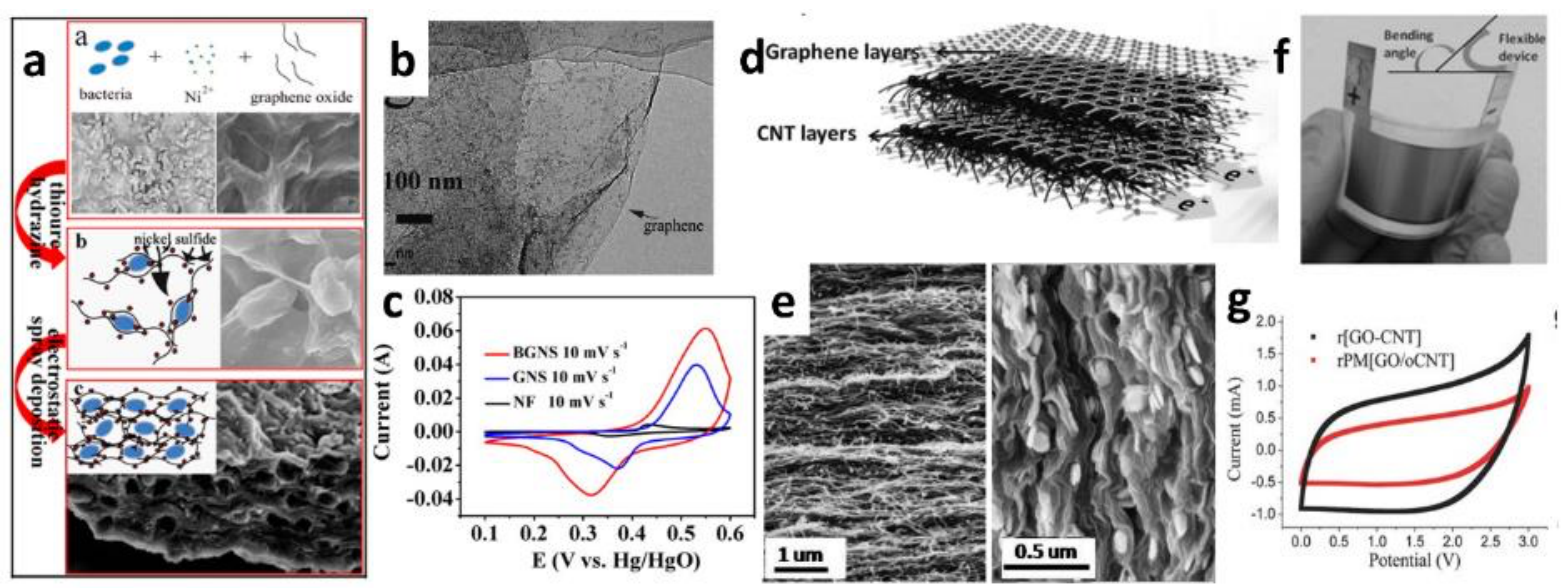

Figure 33: Schematic of the synthesis of $\mathrm{rGO} / \mathrm{Ni}_{3} \mathrm{~S}_{2}$ porous hybrid templated by bacteria and (b) TEM of the hybrid. (c) CV curves of nickel foam, $\mathrm{rGO} / \mathrm{Ni}_{3} \mathrm{~S}_{2}$ and of bacteria/rGO/Ni $3 \mathrm{~S}_{2}$. Adapted from Ref. ${ }^{[142]}$ (d) Schematic of the rGO/CNTs hybrid electrode. (e) SEM images of the $\mathrm{rGO} / \mathrm{CNTs}(\mathrm{left})$ and $\mathrm{rGO} / \mathrm{Ni}(\mathrm{OH})_{2}$ nanoplates (right) electrodes. (f) Photo of the flexible assembled supercapacitor. Adapted from Ref. [36d] (g) CV curves of rGO chemically functionalized with CNTs (black) and rGO/CNTs physically mixed. Adapted from Ref. ${ }^{[36 a]}$

Hybrids of graphene with electrochemically active polymers have also found promising applications as supercapacitor electrodes. Among these polymers, hybrids with polyaniline (PANI) are the foremost explored because of its electrical conductivity and the fast dopingdedoping redox reactions with metallic cations. ${ }^{[74,186,219]}$ In one of the first examples, Wu et $a l .{ }^{[223]}$ prepared a free-standing rGO-PANI flexible electrode prepared by filtration method. The 


\section{WILEY-VCH}

performances of the electrode were tested in an electrochemical cell and showed a specific capacity of $210 \mathrm{~F} \mathrm{~g}^{-1}$ at $0.3 \mathrm{~A} \mathrm{~g}^{-1}$. An et al. ${ }^{[224]}$ prepared a hybrid of rGO chemically functionalized with PANI chains, via amide bond formation between PANI and the acyl chloride derivative of GO. The hybrid was deposited on a glassy carbon electrode and tested as a supercapacitor using a $2 \mathrm{M} \mathrm{H}_{2} \mathrm{SO}_{4}$ aqueous electrolyte. The hybrid exhibited a high specific capacitance of $623.1 \mathrm{~F} \mathrm{~g} \mathrm{~g}^{-1}$ at a current density of $0.3 \mathrm{~A} \mathrm{~g} \mathrm{~g}^{-1}$, and $510 \mathrm{~F} \mathrm{~g}^{-1}$ at a current density of $50 \mathrm{~A} \mathrm{~g}^{-1}$ and long-term cycling durability. In another example, Meng et al. ${ }^{[225]}$ prepared a highly porous free-standing hybrid of $\mathrm{rGO}$ and polyaniline, with the aid of $\mathrm{CaCO}_{3}$ particles as sacrificial templating agent. The polyaniline was polymerized in situ on the porous 3D rGO scaffold (Figure $34 \mathrm{a}-\mathrm{b}$ ). The hybrid presented a specific capacity of $385 \mathrm{~F} \mathrm{~g}^{-1}$ at $0.5 \mathrm{~A} \mathrm{~g}^{-1}$, with $94 \%$ of capacitance retention at $10 \mathrm{~A} / \mathrm{g}$, and excellent cycling stability (90\% capacitance retention after 5000 cycles) (Figure $34 \mathrm{c}$ ). In addition to PANI, hybrids of graphene with other polymers have been tested. Recently our group conceived a pseudocapacitor based on rGO covalently functionalized with a thiourea-formaldehyde polymer (Figure 34 d). ${ }^{[226}$ The hybrid was processed via freeze-drying and presented high porosity. The pseudocapacitor performances were tested in an electrochemical cell and revealed a high specific capacity of $400 \mathrm{~F} \mathrm{~g}^{-1}$ at a scan rate of $1 \mathrm{mV} \mathrm{s}^{-1}$ and $100 \%$ retention of the capacitance after 5000 cycles at $100 \mathrm{mV} \mathrm{s}^{-1}$ (Figure 34 e). Liu et al. ${ }^{[227]}$ fabricated a solid-state micro-supercapacitor ${ }^{\text {based }}$ on a hydrophilic electrochemically exfoliated graphene (EEG) functionalized with polydopamine, The EEG was produced with relatively low oxygen content (12\%) that allowed its dispersibility in water, in which it was functionalized with polydopamine. Then the hybrid material was deposited onto $\mathrm{Si} / \mathrm{SiO}_{2}$ substrates, gold electrodes were evaporated on top and a polymer electrolyte was added. The micro/supercapacitor exhibited high volumetric capacitance and power density of $340 \mathrm{~F} \mathrm{~cm}^{-3}$ and $1000 \mathrm{~W} \mathrm{~cm}^{-3}$, respectively. 


\section{WILEY-VCH}

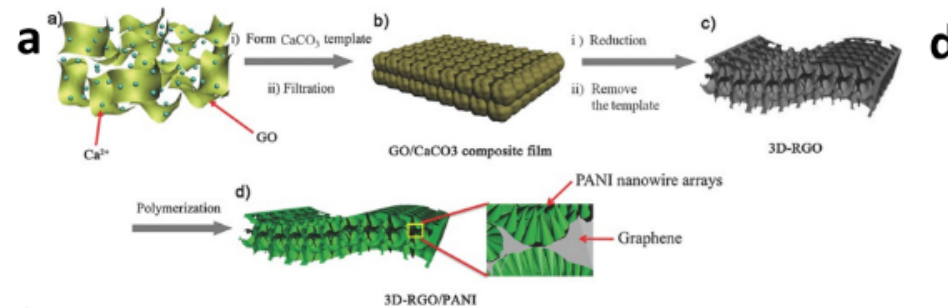

b

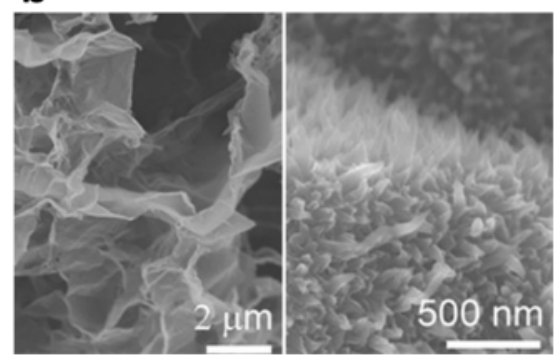

c

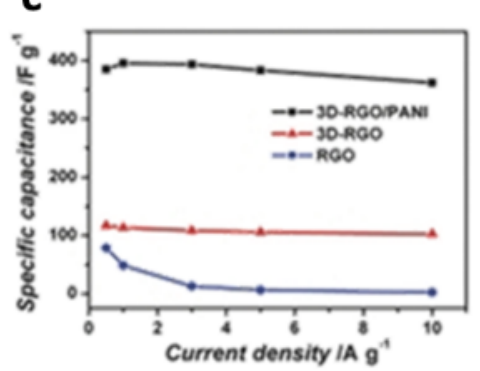

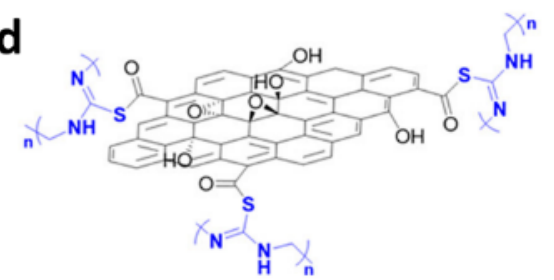

e

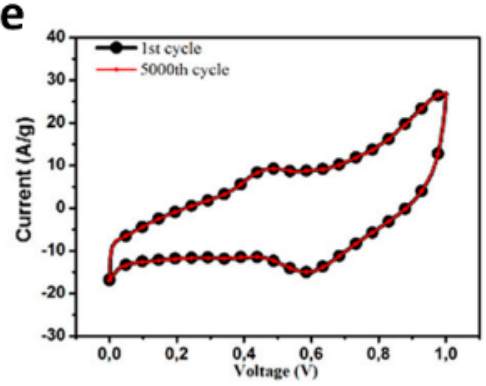

Figure 34: (a) Schematic of synthesis of rGO/PANI hybrid templated by $\mathrm{CaCO}_{3} \mathrm{NPs}$ and (b) SEM images of the same, showing the porosity of the hybrid and the PANI nanofibers. (c) Specific capacitance of $\mathrm{rGO}$, porous $\mathrm{rGO}$ and porous $\mathrm{rGO}$ /PANI. Adapted from Ref. ${ }^{[225]}$ (d) Structure of the hybrid $\mathrm{rGO} /$ thiourea-formaldehyde polymer and (d) $\mathrm{CV}$ of the same at the $1^{\text {st }}$ and $5000^{\text {th }}$ cycle at $100 \mathrm{mV} / \mathrm{s}$ showing the high stability. Adapted from Ref. [226]

\subsection{Graphene-based hybrids as transparent electrodes for optoelectronic}

\section{applications}

The exceptional electronic, optical and mechanical properties of graphene have envisioned its use in optoelectronic devices as transparent conductive electrodes (TCEs). TCEs are fundamental components of several devices, including various kinds of solar cells, display technologies, organic light emitting diodes (OLEDs), touch screens and photodetectors. Nowadays the market standard for TCEs is indium tin oxide, i.e. ITO. This metal oxide, composed in large part by indium, can produce films with transmittances (T) around $93 \%$ in the visible range and sheet resistance $\left(\mathrm{R}_{\mathrm{s}}\right)$ as low as $15-20 \Omega \mathrm{sq}^{-1}$ on glass substrates. ${ }^{[228]}$ Typical sheet resistances of commercially available ITO films are usually higher, especially for ITO on plastic substrates such as PET, in which the sheet resistance can raise up to $100 \Omega \mathrm{sq}^{-1}$. ${ }^{[229]}$ However, ITO suffers of some important drawbacks, which are the high cost and low 


\section{WILEY-VCH}

sustainability due to the presence of the rare earth metal indium, the scarce flexibility and low electrochemical and chemical stability. Pristine single-layer graphene (SLG) is highly transparent, with a transmittance in the visible of $97.7 \%$ and a reflectance of $0.1 \%$, and highly conductive, with charge carrier mobilities in the order of $10^{4} \mathrm{~cm}^{2} \mathrm{~V}^{-1} \mathrm{~s}^{-1}{ }^{[230]}$ These properties make it a promising alternative to ITO. Nowadays, the largest and continuous films of graphene with the best properties in terms of transparency and conductivity are obtained by CVD. However, even the best CVD graphene monolayer presents a $\mathrm{T}=97 \%$ and a sheet resistance of $600-650 \Omega \mathrm{sq}^{-1},{ }^{[230-231]}$ while for multilayer graphene the state of the art figures of merit are $\mathrm{T}=84 \%$ and $\mathrm{R}_{\mathrm{s}}=220 \Omega \mathrm{sq}^{-1} \cdot{ }^{[232]}$ These values are quite far from the performances of ITO films. Films made of overlapped rGO flakes can have sheet resistances as low as $1000 \Omega \mathrm{sq}^{-1}$ at $\mathrm{T}=80 \%$ for films reduced with hydrazine and $800 \Omega \mathrm{sq}^{-1}$ at $\mathrm{T}=82 \%$ for films annealed at $1100{ }^{\circ} \mathrm{C}$ under Ar. ${ }^{[231 b]}$ Despite the higher sheet resistances of rGO films, their principal appeal is due to the lower cost and much easier processability and deposition compared to CVD graphene, which needs to be transferred from the metallic substrate to the device. The sheet resistance is not the only important figure of merit of TCEs, in fact, the electrodes need to efficiently collect and inject electrons in the active material of the device, and to do so they need to have a work function that is properly aligned with the frontier orbitals of the active material. Moreover, in order to increase the conductivity and adjust the work function, CVD graphene and rGO films can be doped with small molecules. ${ }^{[230,231 b]}$ For instance, CVD graphene doped with p-dopant such as $\mathrm{AuCl}_{3}$ and $\mathrm{HNO}_{3}$ can exhibit $\mathrm{R}_{\mathrm{s}}$ as low as $30 \Omega \mathrm{sq}^{-1}$ for $\mathrm{T}=90 \% .{ }^{[230,233]}$ Also rGO can be made more conductive via chemical doping; for example, a rGO film obtained by Langmuir Blodgett (LB) deposition and doped with $\mathrm{SOCl}_{2}$ after reduction

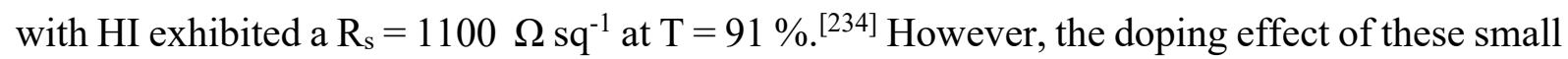
molecules in most of the cases is not stable over time, leading to a degradation of the 


\section{WILEY-VCH}

performances. ${ }^{[230-231]}$ The combination of graphene in hybrid films with bigger aromatic molecules, polymers (and especially conducting polymers) and nanostructures such as metallic nanowires and carbon nanotubes can greatly enhance the conductivity and on the same time maintain a high transparency.

Graphene hybrids have found application in all the types of solar cells, including inorganic semiconductor, perovskite, organic solar cells (OSC) and dye-sensitized solar cells (DSSC). In solar cells, graphene hybrids have been used mainly as ITO replacement in the front TCE, but also as electron acceptor layer and buffer layer in OSC. Solar cells are the devices in which TCEs need the highest transparency and highest conductivity, since these values have a great effect on the power conversion efficiencies (PCEs). Hybrids of CVD graphene and small doping molecules have been used to increase the conductivity of graphene and modulate its work function. For instance, Miao et al. ${ }^{[235]}$ produced a CVD SLG doped with a layer of trifluoromethanesulfonimide and employed it as both TCE and as electron collector in a Schottky junction solar cell with $\mathrm{n}$-Si. The p-doping effect on graphene from trifluoromethanesulfonimide enhanced its conductivity and led to an increase of PCE from 1.9 $\%$ of the un-doped device to $8.6 \%$. Hsu et al. ${ }^{[236]}$ reported the use of a 5 layers structure of alternating single-layer CVD graphene and tetracyanoquinodimethane (TCNQ) as transparent anode for a bulk heterojunction OSC with poly(3-hexylthiophene) (P3HT) and phenyl-C61butyric acid methyl ester (PCBM) (Figure 35 a). The TCNQ sandwiched between two graphene layers act as p-dopant, causing an increase in the work function and a huge reduction of the sheet resistance of the electrode. The overall PCE of the solar cell constructed with the 5 layer graphene/TCNQ electrode was $2.58 \%$, being 6 times higher than that of the device with a single layer CVD graphene electrode (Figure 35 b). Hybrids of graphene with conductive polymers have found application as TCEs in solar cells. In particular, poly(3,4-ethylenedioxythiophene): 


\section{WILEY-VCH}

poly(styrene sulfonic acid) (PEDOT:PSS) has been employed for its p-doping effect and the high intrinsic conductivity. ${ }^{[237]}$ For example, Lee et al. ${ }^{[237 b]}$ presented a TCE based on a bilayer CVD graphene and PEDOT:PSS and used it as top TCE in a polymer-based OSC. In this case, the PEDOT:PSS layer had a dual role: it served as substrate for the transfer of the CVD graphene from the metal to the OSC, and then it enhanced the performance of the device through p-doping. The OSC with graphene/PEDOT:PSS on PET exhibited a PCE of $4.8 \%$, which was even higher than that of the same OSC with a PET/ITO electrode.

Graphene hybrids have also been employed in display technologies, such as OLED and electrochromic devices (ECD), as TCE to replace ITO. In the case of OLED and electrochromic devices, good efficiencies can be obtained also with TCE exhibiting lower transparency in comparison to solar cells. Meyer et al. ${ }^{[238]}$ produced a TCE composed of a CVD graphene fewlayers and a $\mathrm{MoO}_{3}$ layer, thermally evaporated on top Figure 35 c). The $\mathrm{MoO}_{3}$ layer acts as pdopant, lowering the sheet resistance of the film and increasing the work function of graphene Figure 35 d-e). Furthermore, the doping produces an alignment of the transport levels on the two materials, favouring the charge extraction/injection. The TCE was tested in an OLED device, which showed performances slightly superior to that of a similar OLED with glass/ITO electrode. Chang et al. ${ }^{[239]}$ presented a TCE based on a hybrid of rGO and PEDOT:PSS as addictive for increasing the conductivity. GO was exfoliated and reduced in solution with the aid of sodium dodecylbenzene sulfonate as a surfactant and then it was mixed with a solution of PEDOT:PPS and spin-coated on PET substrate. The PEDOT:PSS greatly improved the conductivity of rGO by acting as a dopant and as a conductive filler that occupied all the empty spaces between the rGO flakes. The hybrid film was used as a transparent anode in an OLED, which performances were comparable with that of an OLED made with an ITO anode. 


\section{WILEY-VCH}

a

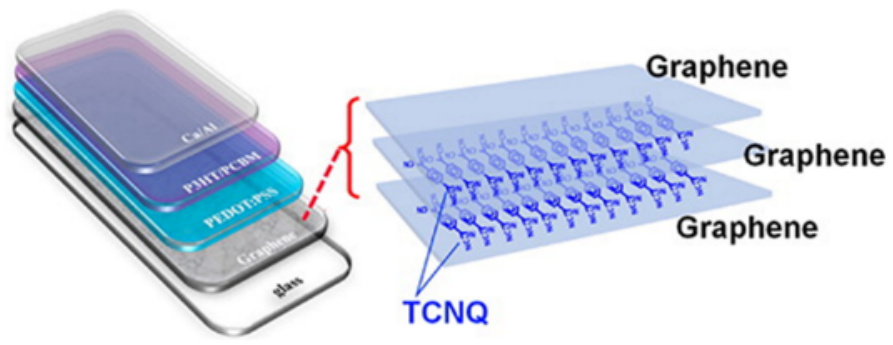

C

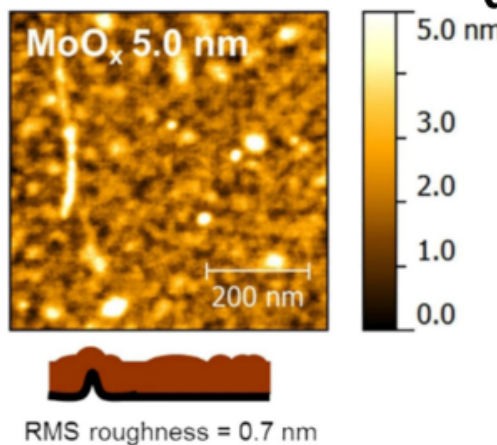

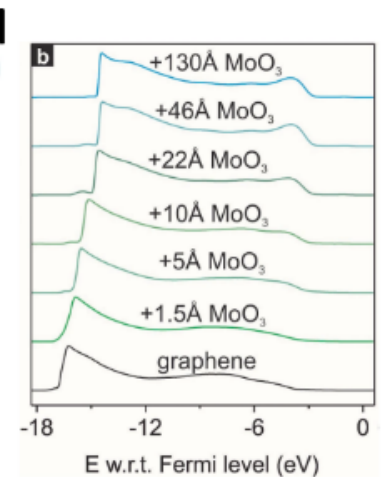
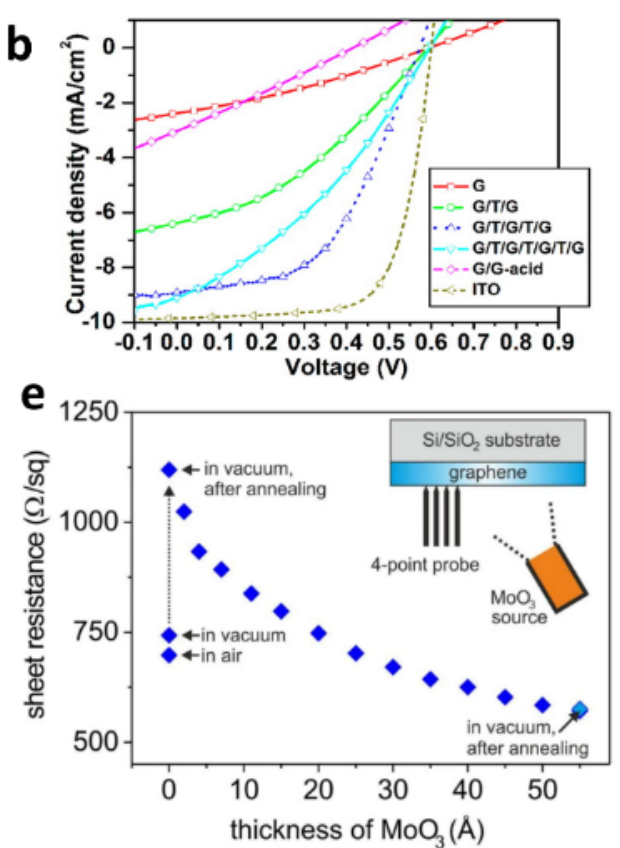

Figure 35: (a) Schematic structure of a OSC with a TCE based on a sandwiched structure of CVD graphene monolayer and TCNQ and (b) Measured current density (J)-applied bias (V) curves of the OSC devices with different TCEs. Adapted from Ref. ${ }^{[236]}$ (c) AFM image of the hybrid monolayer CVD graphene/ $\mathrm{MoO}_{3}$ electrode. (d) UPS spectra plotted with respect to Fermi level of graphene with incrementally deposited $\mathrm{MoO}_{3}$ layers. (e) Sheet resistance measurements of monolayer graphene. Measurement sequence: in air, in vacuum, after annealing at $140^{\circ} \mathrm{C}$ in vacuum, upon deposition of $\mathrm{MoO}_{3}$, after annealing at $140^{\circ} \mathrm{C}$. Adapted from Ref. ${ }^{[238]}$

Carbon nanotubes, Ag NWs and $\mathrm{Cu}$ NWs have also been used in order to increase the conductivity of rGO and CVD graphene. Tung et al. ${ }^{[35 \mathrm{~d}]}$ produced a TCE made of a hybrid rGO/CNTs. The two components were mixed in solution, reduced with hydrazine, and deposited by spin coating on a PET substrate. The electrode exhibited a $R_{\mathrm{s}}$ of $240 \Omega \mathrm{sq}^{-1}$ at 86 $\%$ transmittance. The hybrid film was used as top electrode in a P3HT/PCBM OSC, which exhibited a PCE of $0.85 \%$. By virtue of its good stability, a rGO/CNTs TCE was also used by Zhu et al. ${ }^{[240]}$ to substitute platinum as counter electrode for DSSC. Ruoff et al. ${ }^{[241]}$ prepared a hybrid TCE composed of a layer of aligned metallic multi-walled CNTs coated by a continuous layer of CVD graphene deposited on top of a PET substrate. The electrode, which exhibited high bendability, was tested as top and bottom electrode in an ECD, showing good performances (Figure 36 a-c). Interestingly, the inverted configuration of the electrode with 


\section{WILEY-VCH}

the CNTs on top of graphene showed worse electrical, optical and mechanical performances. Ag NWs and $\mathrm{Cu}$ NWs produce randomly distributed 2D networks possessing high transparency and conductivity, however their practical application is hindered by their low stability. Graphene (including rGO) can be used to insulate the nanowires from the external environment, protecting them from the oxidation and in the meanwhile, preserving their conductivity. Ricciardulli et al. ${ }^{[43 \mathrm{a}]}$ prepared a hybrid TCE of Ag NWs/electrochemically exfoliated graphene (EEG) on different substrates via spray-coating of the two components from suspension. The EEG coating on top of the Ag NWs greatly decreased the roughness of the film and the sheet resistance from 78 to $13.7 \Omega$ /sq, and increased their stability to oxidation and to mechanical bending (Figure 35 d). The hybrid film was integrated as top electrode in a bulk heterojunction OSC, which exhibited a PCE of $6.57 \%$ (Figure 35 e). Dong et al. ${ }^{[43 \mathrm{~b}]}$ prepared a TCE composed by Ag NWs network covered by CVD SLG. The hybrid electrode was prepared by spin coating of the Ag NWs on top of the transferred CVD graphene, followed by inversion of the structure via a second transfer step. Also in this case, the overlying graphene layer increased the resistance to corrosion of the electrode, reduced the roughness and lowered the sheet resistance $\left(R_{\mathrm{S}}=8.06 \Omega \mathrm{sq}^{-1}\right.$ at $\left.\mathrm{T}_{(550 \mathrm{~nm})}=88.3 \%\right)$. The TCE was used as top electrode in a perovskite solar cell and outperformed a similar device with a commercial PET/ITO electrode. Aliprandi et al. ${ }^{[35 f]}$ prepared a hybrid $\mathrm{rGO} / \mathrm{CuNWs}$ transparent electrode which was successfully used to replace ITO in a flexible electrochromic device. An ethanolic dispersion of $\mathrm{Cu}$ NWs was spray-coated on PET substrate, followed by the spray-coating of a GO ink and the simultaneous reduction of both $\mathrm{Cu}$ NWs and rGO by a diluted $\mathrm{NaBH}_{4}$ solution. The electrode exhibited a sheet resistance $<30 \Omega \mathrm{sq}^{-1}$ at $\mathrm{T}_{(550 \mathrm{~nm})}>70 \%$, high stability to bending and to the exposure to air. Dou et al. reported a solution-based approach to coat the $\mathrm{Cu}$ NWs with a self-assembled layer of GO nanosheets. ${ }^{[242]}$ Then the wrapped Cu NWs@GO were 


\section{WILEY-VCH}

deposited onto a substrate, followed by a mild thermal annealing to reduce GO, yielding a conducting network. The conducting film exhibited a sheet resistance of $14.8 \Omega \mathrm{sq}^{-1}$ at transmittance of $86.5 \%$.
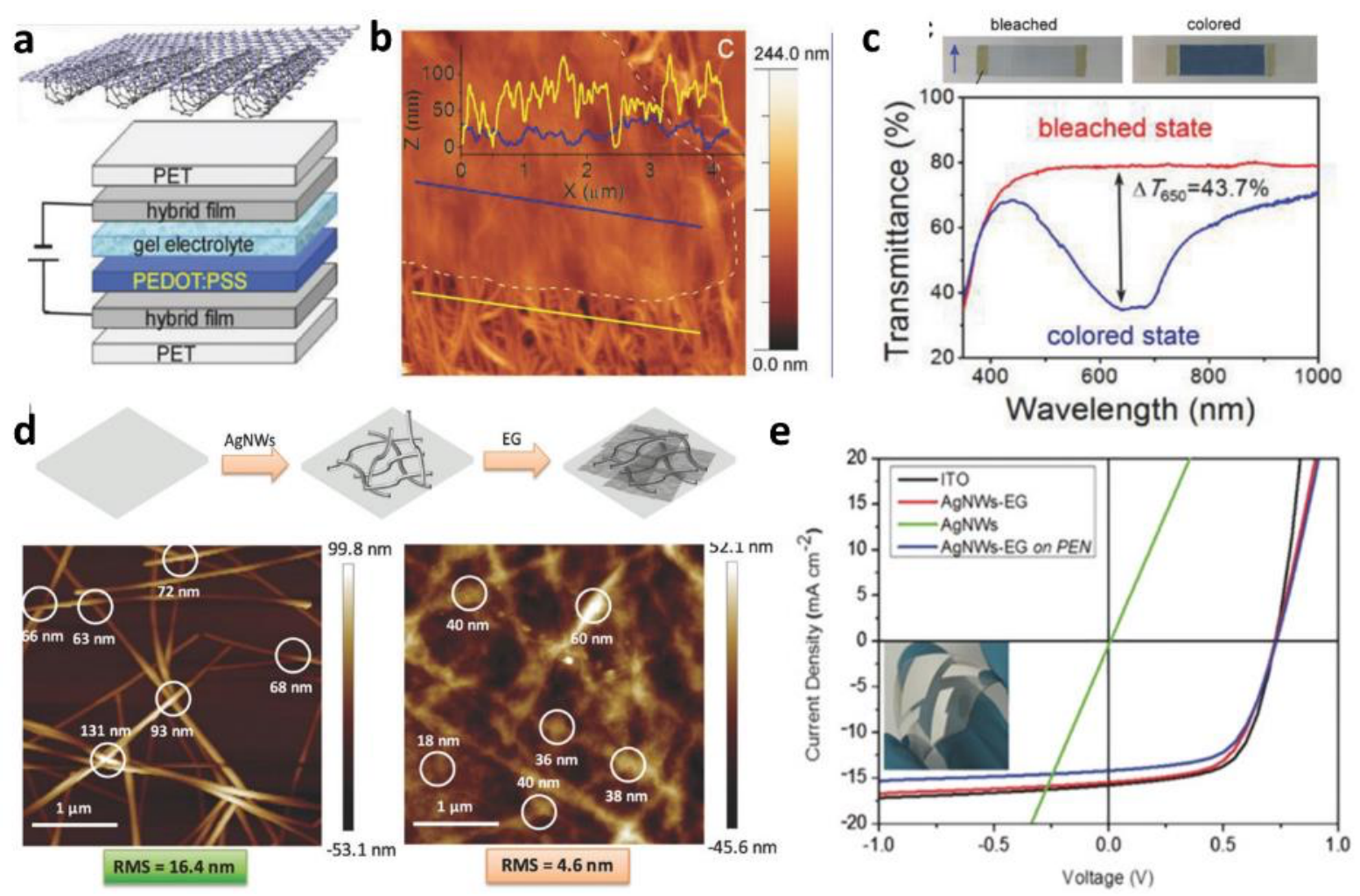

Figure 36: (a) Schematic of the electrode CNTs/SLG and of the ECD device. (b) AFM image of the CNTs partially covered by SLG and height profiles of CNTs and CNTs/SLG. (c) Photos and Transmission spectra of the ECD device in the coloured and bleached states. Adapted from Ref. ${ }^{[241]}$ (d) Top: schematic of the fabrication of the hybrid TCE Ag NWs/EEG. Bottom: AFM images with reported the roughness (RMS) of Ag NWs and Ag NWs/EEG on glass. (e) J - V curves of the OSC device assembled with different top TCE: ITO, Ag NWs, Ag NWs/EEG on glass and PEN. Adapted from Ref. ${ }^{[43 a]}$

\subsection{Photodetectors}

A photodetector is a transducer that absorbs photons and converts them into an electrical signal.

The electrical signal generated can be a photocurrent, a difference of potential or a change in resistance. Graphene has been intensively investigated for its photonic properties and its first 


\section{WILEY-VCH}

application as photodetector dates back to $2009 .{ }^{[243]}$ A single graphene layer absorbs as much as $2.3 \%$ of light, being a notably large magnitude for an atom-thick material. Moreover, its light absorption is wavelength independent. Other characteristics that make graphene so appealing for photonic applications are its zero bandgap, which allows graphene to absorb photons and generate charge carriers over an extremely wide range of frequencies, from ultraviolet to microwaves, and its high carrier mobility, which allows an extremely fast detection. ${ }^{[244]}$ However, the low absorption of monolayer graphene implies that most of the incident photons do not interact with graphene. Furthermore, the zero bandgap of graphene causes a superfast charge-recombination in the ps range, which one the one hand is favourable for high-speed optoelectronics, and on the other hand, makes it difficult to separate and collect the photo-generated carriers. For these reasons, graphene is often coupled with other nanostructures, such as few layers TMDs, and QDs, which absorbs the photons and generate a hole and an electron that are efficiently collected by graphene. ${ }^{[46]}$ In these cases graphene act as a transparent electrode, enabling the light to pass through and collecting and transporting the photogenerated carriers. For example, in sandwiched vertical heterostructures Graphene/TMD/Graphene the graphene layers allow the light to pass and collect immediately the photogenerated carrier in the TMD without long diffusion process. For example, Duan and co-workers ${ }^{[135 a]}$ developed a vertical heterostructure composed of a mechanically exfoliated 50 nm thick $\mathrm{MoS}_{2}$ sandwiched between two CVD SLGs deposited on a $\mathrm{Si} / \mathrm{SiO}_{2}$ substrate (Figure 37 a-b). The two interfaces graphene/MoS 2 produce two Schottky barriers and since the $\mathrm{MoS}_{2}$ layer is thin, the two Schottky barriers merge together and form a monotonic band slope (Figure 37 e). Excitons formed in the $\mathrm{MoS}_{2}$ are separated and immediately collected by the graphene electrodes. The height of the Schottky barriers, and thus the slope of the $\mathrm{MoS}_{2}$ bands, can be modulated by changing the doping of the bottom graphene by the application of a back gate 


\section{WILEY-VCH}

voltage to the bottom graphene electrode, eventually leading to the modulation of the photoresponse (Figure $37 \mathrm{~d}-\mathrm{e}$ ). By decreasing the thickness of the TMD, it is possible to increase the detection speed of the device, at expenses of the sensitivity. ${ }^{[101]}$
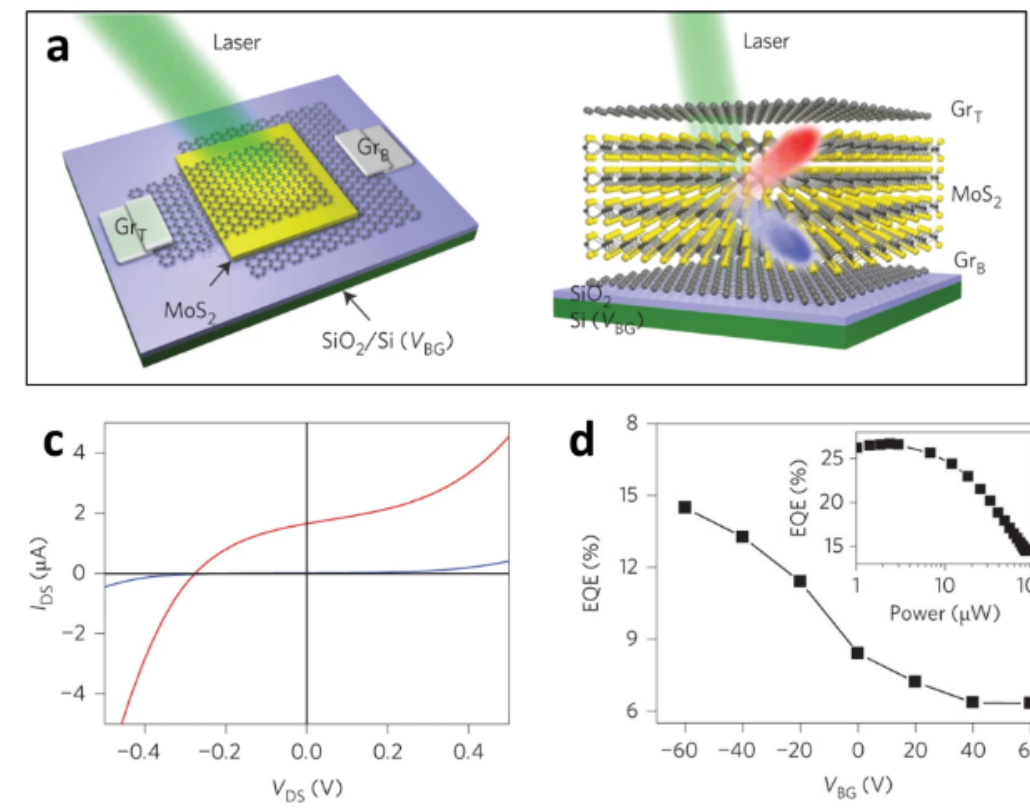

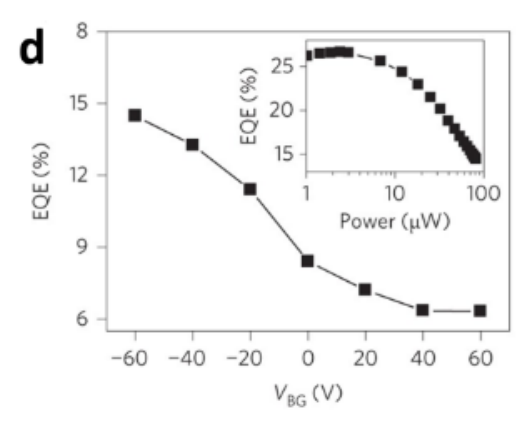

b

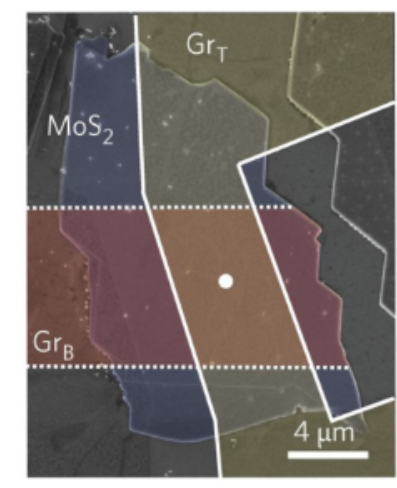

e

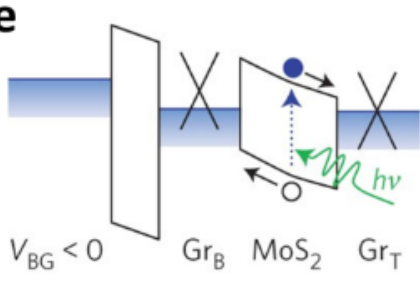

Figure 37: (a) Schematic representation of the photodetector device SLG/MoS $/$ SLG view from the top and from the side and (b) SEM image in false colours of the device. (c) Current-voltage characteristic of the device in dark (blue) and under illumination (red) by a focused laser beam $(\lambda=514 \mathrm{~nm} ; 80 \mu \mathrm{W}$; spot size $=1 \mu \mathrm{m})$. External quantum efficiency as a function of the backgate Voltage $\left(V_{B G}\right)$ at a laser power of $80 \mu \mathrm{W}$ and of the excitation laser power at $V_{B G}=60 \mathrm{~V}$. (e) Schematic band diagrams of the vertical heterostructure with negative $V_{B G}$.

In order to increase the sensitivity up to the single photon detection, graphene is often coupled with photosensitive materials which absorb light efficiently, such as quantum dots. ${ }^{[101,231 a, 244]}$ In these cases, usually the incident light is detected by photogating effect: photons are absorbed by the photosensitizer and generate excitons, which are separated by the in-built electrical field at the interface between graphene and the photosensitizer. Eventually, one type of the charges (hole or electron) is collected by graphene, and the opposite charges remain trapped into the photosensitizer, altering the gate voltage. The longer is the lifetime of the trapped charges and the higher is the mobility of the collected charges in graphene, the higher is the modulation of the gate voltage, and thus the sensitivity. Konstantatos et al. ${ }^{[135 b]}$ presented an ultrasensitive 


\section{WILEY-VCH}

photodetector based on the photogating effect, composed by a mechanically exfoliated SLG on a $\mathrm{Si} / \mathrm{SiO}_{2}$ substrate coated by a $80 \mathrm{~nm}$ thick layer of $\mathrm{PbS}$ colloidal QDs, produced by $\mathrm{LbL}$ deposition. The absorption of photons was detected both as a shift in the Dirac point in graphene towards more negative back gate voltages and as a decrease in the resistivity of graphene. The device exhibited great light sensitivity, being able to detect light as little as $8 \mathrm{fW}$ of incident light (Figure 38 a-c). The spectral range of sensitivity of photosensitized graphene photodetectors can be modulated by changing the QDs and their optical characteristics. QDs are sensitive to the incident light if its energy is higher than the bandgap of the QDs. Bigger QDs have narrower bandgaps and absorb light from higher wavelengths. Ni et al. ${ }^{[245]}$ produced a photodetector sensitive from the mid-infrared (MIR) to UV light by depositing B-doped Si QDs on CVD graphene on a $\mathrm{Si} / \mathrm{SiO}_{2}$ substrate. MIR light is directly absorbed by graphene, which absorption in this region is enhanced by the localized surface plasmon resonance (LSPR) induced by the Si QDs. The light with wavelengths from near-infrared to UV is absorbed by the QDs and is detected by photogating effect. The devices exhibited ultrahigh responsivity up to $10^{9} \mathrm{~A} \mathrm{~W}^{-1}$ (Figure $38 \mathrm{~d}-\mathrm{e}$ ). The photosensitizing effect may arise also from organic photosensitizers, such as porphyrins. ${ }^{[135]}$ For example, Wang et al. ${ }^{[246]}$ produced a photodetector based on a hybrid of mechanically exfoliated SLG coated by drop-cast chlorophyll A molecules, which acted as photosensitizer. The photodetector displayed a high responsivity of $10^{6} \mathrm{~A} \mathrm{~W}^{-1}$. Hybrids of graphene with QDs can be obtained also by solution processing. For example, Manga et al. ${ }^{[247]}$ produced a broad-band sensitive photodetector based on a hybrid of few-layer electrochemically exfoliated graphene decorated by $\mathrm{PbSe}$ and $\mathrm{TiO}_{2}$ QDs, synthesized in situ on the graphene surface. The hybrid material was inserted between a transparent ITO electrode and an Al electrode (Figure $38 \mathrm{f}$ ). The photodetector was sensitive from IR to UV region; however, the maximum responsivity amounted only to $0.506 \mathrm{~A} \mathrm{~W}^{-1}$. 


\section{WILEY-VCH}
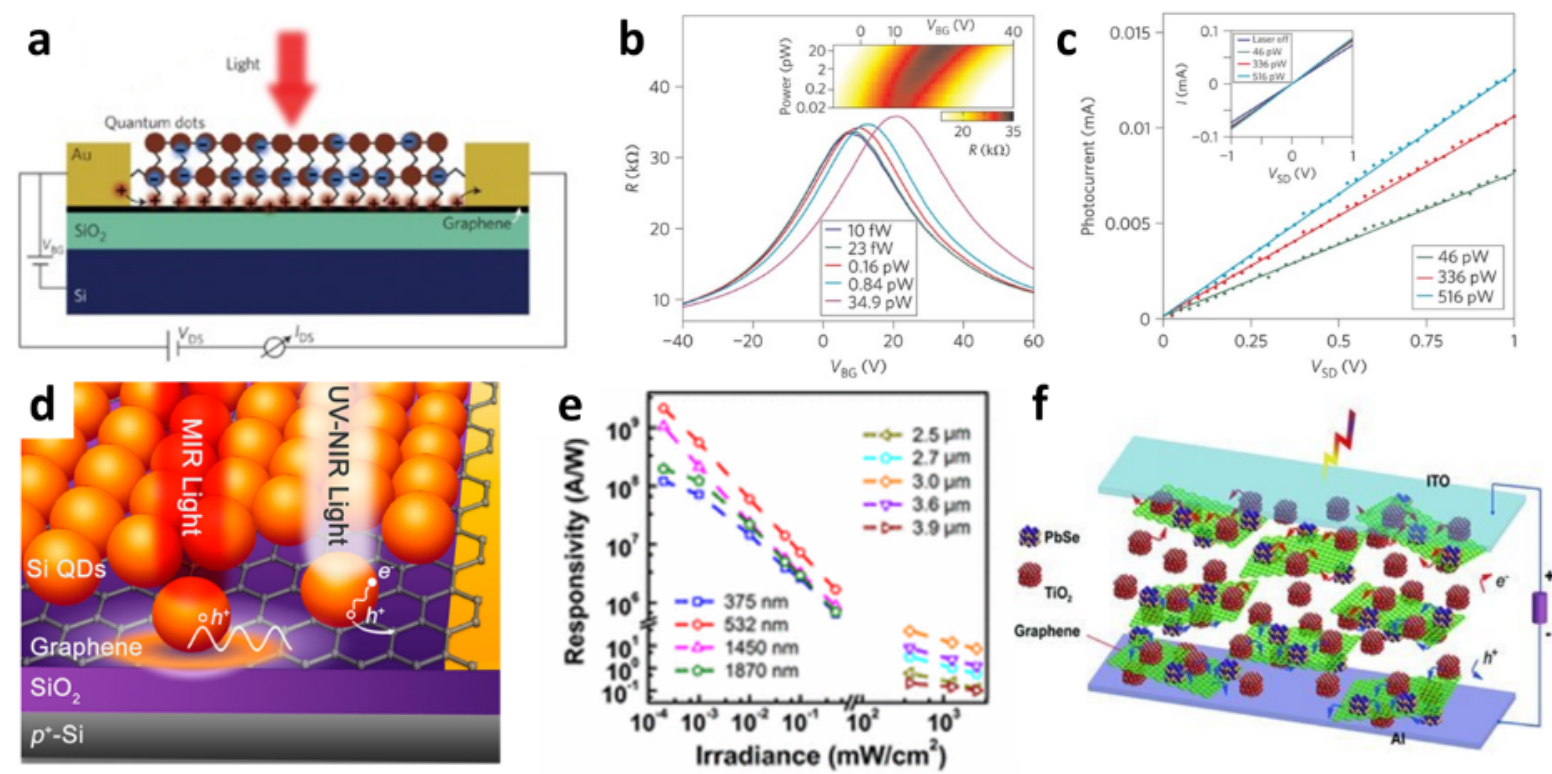

Figure 38: (a) Schematic of the SLG/PbS QDs hybrid photodetector. (b) Resistance vs $\mathrm{V}_{\mathrm{BG}}$ of the device at different illumination intensities of a collimated laser $(\lambda=500 \mathrm{~nm}$, diameter $=$ $1 \mathrm{~mm}$ ) showing the modulation of the Dirac point. (c) Photocurrent as a function of drain-source voltage $\left(\mathrm{V}_{\mathrm{DS}}\right)$ at $\mathrm{V}_{\mathrm{DG}}=0$ of the SLG/PbS QDs photodetector for different optical powers. Adapted from Ref. ${ }^{[135 b]}$ (d) Schematic of the SLG/B-doped Si QDs photodetector and (e) Responsivity of the device as a function of the irradiance and of the incident light wavelength. Adapted from Ref. ${ }^{[25]}$ (f) Schematic of the photodetector based on a hybrid FLG functionalized with $\mathrm{PbSe}$ and $\mathrm{TiO}_{2}$ QDs. Adapted from Ref. ${ }^{[247]}$

\section{Conclusion and perspectives}

In this review we have summarized the most enlightening approaches reported during the last 15 years on the generation of functional hybrid structures composed by graphene (including GO and $\mathrm{rGO}$ ) and other nanostructures or molecules. We have described the main synthetic strategies, the emergence of new properties and the application of these hybrids, with a particular focus on their different, and often superior, performances compared to their nothybridized counterpart. The use of graphene as a building block to assemble more complex, functional supramolecular architectures and its functionalization with molecules was proven being a successful route to tailor the functionalities of graphene and multiply its potential applications in the most disparate fields. Hitherto, with over 35000 publications, graphene- 


\section{WILEY-VCH}

based hybrid assemblies represent the $28 \%$ of the total number of publications comprising graphene (Figure 39 a). In particular, while the gold rush of graphene seems to be slowly reaching its peak, graphene-based hybrids are still steadily growing (Figure 39 b). However, despite the great number of publications, the graphene-based hybrid structures struggle to find their use in industrial end products.
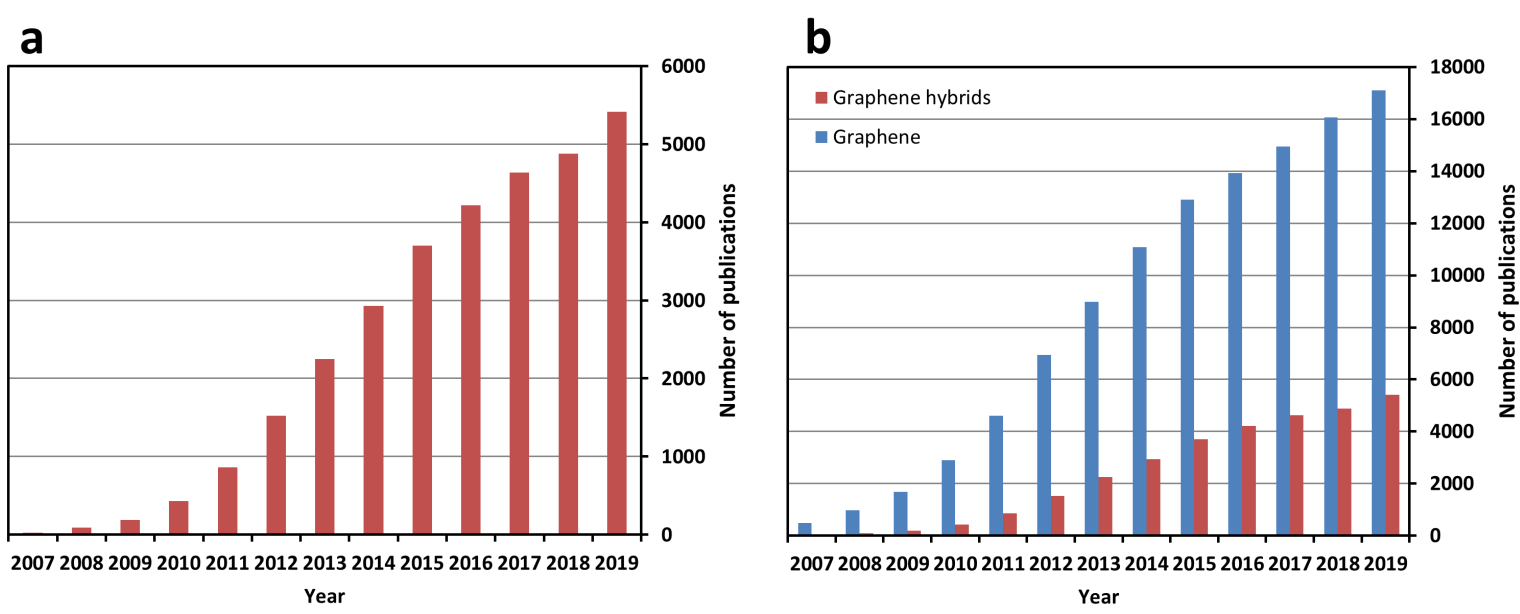

Figure 39: (a) Number of publications with subject graphene-based hybrid as a function of year and (b) comparison with the publications with subject graphene. Source: Web of Science. The results for graphene-based hybrids have been refined with the following query: $\mathrm{TI}=$ (graphene AND (hybrid OR nanocomposite OR functionaliz* OR nanostructur*)) OR AB=(graphene AND (hybrid OR nanocomposite OR functionaliz*)) AND TI=(graphene OR RGO)

On the contrary, composites materials in which graphene is used as nanofiller have already emerged in several products on the market. ${ }^{[248]}$ The main reason is that these composites materials use cheap forms of graphene, usually obtained by liquid-phase exfoliation of graphite, which often are nothing but micrometre sized thin graphite flakes. ${ }^{[249]}$ These composites usually display enhanced mechanical properties, electrical and thermal conductivities and reduced weight; however, the performances of these composites do not stand out compared to that of other composite materials. ${ }^{[250]}$ Before seeing graphene-based hybrids into the market, few challenges still need to be tackled. In particular, graphene, GO and rGO are still expensive material, and their quality is highly dependent on the production method used. Furthermore, the 


\section{WILEY-VCH}

synthetic procedures used for preparing the hybrids are often complexes and scarcely reproducible. Therefore, to meet the industrial requirements it is necessary a cost reduction, an optimization of the synthetic procedures that guarantees a higher reproducibility and an improvement of the long-term stability of the hybrids. Nevertheless, we believe that we are just at the early stage of the research on these materials, thus there is still plenty of room for improving the performances, reducing the costs and ultimately allowing products with a revolutionary impact in the years to come. New technologies indeed necessitate several decades for implanting into society. ${ }^{[251]}$ For example, it took 15 years for silicon-based semiconductors to appear in the first commercial products, and more than 20 years to completely supplant other existing technologies. ${ }^{[252]}$ We believe that graphene-based hybrids possess the qualities to emerge as disruptive technologies in optoelectronics, water purification, energy, biomedical applications, as well as sensing, with the ultimate goal of improving the quality of life by providing a decisive step forward in the major challenges our society is facing, i.e. energy, water and food, environment and climate change, and health care.

\section{Acknowledgements}

We acknowledge funding from European Commission through the ERC project SUPRA2DMAT (GA-833707), the Graphene Flagship Core 3 project (GA-881603), the H2020 DECOCHROM project (GA-760973), the Agence Nationale de la Recherche through the Labex projects CSC (ANR-10-LABX-0026 CSC) and NIE (ANR-11-LABX-0058 NIE) within the Investissement d'Avenir program (ANR-10-120 IDEX-0002-02), the International Center for Frontier Research in Chemistry (icFRC) as well as the Institut Universitaire de France (IUF).

\section{References}




\section{WILEY-VCH}

[1] J. D. Bernal, Proceedings of the Royal Society of London. Series A, Containing Papers of a Mathematical and Physical Character 1924, 106, 749.

[2] K. S. Novoselov, A. K. Geim, S. V. Morozov, D. Jiang, Y. Zhang, S. V. Dubonos, I. V. Grigorieva, A. A. Firsov, Science 2004, 306, 666.

[3] A. K. Geim, K. S. Novoselov, Nat. Mater. 2007, 6, 183.

[4] M. D. Stoller, S. J. Park, Y. W. Zhu, J. H. An, R. S. Ruoff, Nano Lett. 2008, 8, 3498.

[5] A. S. Mayorov, R. V. Gorbachev, S. V. Morozov, L. Britnell, R. Jalil, L. A. Ponomarenko, P. Blake, K. S. Novoselov, K. Watanabe, T. Taniguchi, A. K. Geim, Nano Lett. 2011, 11, 2396.

[6] A. A. Balandin, S. Ghosh, D. Teweldebrhan, I. Calizo, W. Bao, F. Miao, C. N. Lau, 2008 Ieee Silicon Nanoelectron. Workshop 2008, 161.

[7] C. Lee, X. Wei, J. W. Kysar, J. Hone, Science 2008, 321, 385.

[8] R. R. Nair, P. Blake, A. N. Grigorenko, K. S. Novoselov, T. J. Booth, T. Stauber, N. M. R. Peres, A. K. Geim, Science 2008, 320, 1308.

[9] A. Ciesielski, P. Samori, Chem. Soc. Rev. 2014, 43, 381.

[10] A. M. Abdelkader, A. J. Cooper, R. A. W. Dryfe, I. A. Kinloch, Nanoscale 2015, 7, 6944.

[11] S. Gilje, S. Han, M. Wang, K. L. Wang, R. B. Kaner, Nano Lett. 2007, 7, 3394.

[12] Y. Wang, Y. Chen, S. D. Lacey, L. Xu, H. Xie, T. Li, V. A. Danner, L. Hu, Mater. Today 2018, 21, 186.

[13] C. J. Shearer, A. Cherevan, D. Eder, Adv. Mater. 2014, 26, 2295.

[14] J. Phiri, P. Gane, T. C. Maloney, Mater. Sci. Eng., B 2017, 215, 9.

[15] a) F. Valorosi, E. De Meo, T. Blanco-Varela, B. Martorana, A. Veca, N. Pugno, I. A. Kinloch, G. Anagnostopoulos, C. Galiotis, F. Bertocchi, J. Gomez, E. Treossi, R. J. Young, V. Palermo, Compos. Sci. Technol. 2020, 185, 107848; b) A. J. Marsden, D. G. Papageorgiou, C. Vallés, A. Liscio, V. Palermo, M. A. Bissett, R. J. Young, I. A. Kinloch, 2d Mater 2018, 5, 032003.

[16] V. Georgakilas, J. N. Tiwari, K. C. Kemp, J. A. Perman, A. B. Bourlinos, K. S. Kim, R. Zboril, Chem. Rev. 2016, 116, 5464.

[17] D. A. Giljohann, D. S. Seferos, W. L. Daniel, M. D. Massich, P. C. Patel, C. A. Mirkin, Angew. Chem., Int. Ed. 2010, 49, 3280.

[18] D. V. Talapin, E. V. Shevchenko, Chem. Rev. 2016, 116, 10343. 


\section{WILEY-VCH}

[19] P. N. Njoki, I. I. S. Lim, D. Mott, H.-Y. Park, B. Khan, S. Mishra, R. Sujakumar, J. Luo, C.-J. Zhong, J. Phys. Chem. C 2007, 111, 14664.

[20] D. Thangaraju, R. Karthikeyan, N. Prakash, S. Moorthy Babu, Y. Hayakawa, Dalton Trans. 2015, 44, 15031.

[21] V. Chandra, J. Park, Y. Chun, J. W. Lee, I.-C. Hwang, K. S. Kim, ACS Nano 2010, 4, 3979.

[22] F.-X. Xiao, J. Miao, B. Liu, J. Am. Chem. Soc. 2014, 136, 1559.

[23] S. Mao, S. Cui, G. Lu, K. Yu, Z. Wen, J. Chen, J. Mater. Chem. 2012, 22, 11009.

[24] a) D. Eder, A. H. Windle, J. Mater. Chem. 2008, 18, 2036; b) E. Yoo, T. Okata, T. Akita, M. Kohyama, J. Nakamura, I. Honma, Nano Lett. 2009, 9, 2255.

[25] a) H. Conrad, G. Ertl, E. E. Latta, Surf. Sci. 1974, 41, 435; b) A. Kaniyoor, R. Imran Jafri, T. Arockiadoss, S. Ramaprabhu, Nanoscale 2009, 1, 382; c) M. G. Chung, D.-H. Kim, D. K. Seo, T. Kim, H. U. Im, H. M. Lee, J.-B. Yoo, S.-H. Hong, T. J. Kang, Y. H. Kim, Sens. Actuator B-Chem. 2012, 169, 387; d) T. Zeng, X.-1. Zhang, Y.-r. Ma, H.-y. Niu, Y.-q. Cai, J. Mater. Chem. 2012, 22, 18658; e) L. Yang, W. Luo, G. Cheng, ACS Appl. Mater. Interfaces 2013, 5, 8231.

[26] a) L. Zhou, F. Shen, X. Tian, D. Wang, T. Zhang, W. Chen, Nanoscale 2013, 5, 1564; b) G. Singh, A. Choudhary, D. Haranath, A. G. Joshi, N. Singh, S. Singh, R. Pasricha, Carbon 2012, 50, 385; c) R. K. Mishra, S. B. Upadhyay, A. Kushwaha, T.-H. Kim, G. Murali, R. Verma, M. Srivastava, J. Singh, P. P. Sahay, S. H. Lee, Nanoscale 2015, 7, 11971; d) Q. Huang, D. Zeng, H. Li, C. Xie, Nanoscale 2012, 4, 5651; e) R. K. Mishra, S. B. Upadhyay, A. Kushwaha, T. H. Kim, G. Murali, R. Verma, M. Srivastava, J. Singh, P. P. Sahay, S. H. Lee, Nanoscale 2015, 7, 11971.

[27] a) C. Anichini, W. Czepa, D. Pakulski, A. Aliprandi, A. Ciesielski, P. Samorì, Chem. Soc. Rev. 2018, 47, 4860; b) Z. S. Qian, X. Y. Shan, L. J. Chai, J. R. Chen, H. Feng, Biosens. Bioelectron. 2015, 68, 225; c) H. Dong, W. Gao, F. Yan, H. Ji, H. Ju, Anal. Chem. 2010, 82, 5511.

[28] X. J. Lv, W. F. Fu, H. X. Chang, H. Zhang, J. S. Cheng, G. J. Zhang, Y. Song, C. Y. Hu, J. H. Li, J. Mater. Chem. 2012, 22, 1539.

[29] K. C. Kemp, H. Seema, M. Saleh, N. H. Le, K. Mahesh, V. Chandra, K. S. Kim, Nanoscale 2013, 5, 3149.

[30] N. Alegret, A. Criado, M. Prato, Curr. Med. Chem. 2017, 24, 529. 


\section{WILEY-VCH}

[31] a) X. Shi, H. Gong, Y. Li, C. Wang, L. Cheng, Z. Liu, Biomaterials 2013, 34, 4786; b) K. Yang, L. Hu, X. Ma, S. Ye, L. Cheng, X. Shi, C. Li, Y. Li, Z. Liu, Adv. Mater. 2012, 24, 1868.

[32] Y. Hu, J. O. Jensen, W. Zhang, Y. Huang, L. N. Cleemann, W. Xing, N. J. Bjerrum, Q. Li, ChemSusChem 2014, 7, 2099.

[33] R. Ghosh Chaudhuri, S. Paria, Chem. Rev. 2011, 112, 2373.

[34] J. Zhu, S. Wei, H. Gu, S. B. Rapole, Q. Wang, Z. Luo, N. Haldolaarachchige, D. P. Young, Z. Guo, Environ. Sci. Technol. 2011, 46, 977.

[35] a) J. Kim, M.-S. Lee, S. Jeon, M. Kim, S. Kim, K. Kim, F. Bien, S. Y. Hong, J.-U. Park, Adv. Mater. 2015, 27, 3292; b) I. N. Kholmanov, C. W. Magnuson, A. E. Aliev, H. Li, B. Zhang, J. W. Suk, L. L. Zhang, E. Peng, S. H. Mousavi, A. B. Khanikaev, R. Piner, G. Shvets, R. S. Ruoff, Nano Lett. 2012, 12, 5679; c) M.-S. Lee, K. Lee, S.-Y. Kim, H. Lee, J. Park, K.-H. Choi, H.-K. Kim, D.-G. Kim, D.-Y. Lee, S. Nam, J.-U. Park, Nano Lett. 2013, 13, 2814; d) V. C. Tung, L.-M. Chen, M. J. Allen, J. K. Wassei, K. Nelson, R. B. Kaner, Y. Yang, Nano Lett. 2009, 9, 1949; e) I. N. Kholmanov, S. H. Domingues, H. Chou, X. Wang, C. Tan, J.-Y. Kim, H. Li, R. Piner, A. J. G. Zarbin, R. S. Ruoff, ACS Nano 2013, 7, 1811; f) A. Aliprandi, T. Moreira, C. Anichini, M. A. Stoeckel, M. Eredia, U. Sassi, M. Bruna, C. Pinheiro, C. A. Laia, S. Bonacchi, Adv. Mater. 2017, 29, 1703225; g) Q. Zhang, Y. Di, C. M. Huard, L. J. Guo, J. Wei, J. Guo, J. Mater. Chem. C 2015, 3, 1528.

[36] a) N. Jung, S. Kwon, D. Lee, D.-M. Yoon, Y. M. Park, A. Benayad, J.-Y. Choi, J. S. Park, Adv. Mater. 2013, 25, 6854; b) D. Yu, L. Dai, J. Phys. Chem. Lett. 2009, 1, 467; c) Q. Cheng, J. Tang, J. Ma, H. Zhang, N. Shinya, L.-C. Qin, Phys. Chem. Chem. Phys. 2011, 13, 17615; d) M. Li, Z. Tang, M. Leng, J. Xue, Adv. Funct. Mater. 2014, 24, 7495.

[37] a) Z. Li, Q. He, X. Xu, Y. Zhao, X. Liu, C. Zhou, D. Ai, L. Xia, L. Mai, Adv. Mater. 2018, 30, 1804089; b) P. Xue, S. Liu, X. Shi, C. Sun, C. Lai, Y. Zhou, D. Sui, Y. Chen, J. Liang, Adv. Mater. 2018, 30, 1804165; c) B. Wang, X. Li, X. Zhang, B. Luo, M. Jin, M. Liang, S. A. Dayeh, S. T. Picraux, L. Zhi, ACS Nano 2013, 7, 1437.

[38] a) S. Woo, Y. R. Kim, T. D. Chung, Y. Piao, H. Kim, Electrochim. Acta 2012, 59, 509; b) Q. He, J. Liu, X. Liu, G. Li, D. Chen, P. Deng, J. Liang, Electrochim. Acta 2019, 296, 683; c) S. Chen, Y. Wei, S. Wei, Y. Lin, L. Liu, ACS Appl. Mater. Interfaces 2016, 8, 


\section{WILEY-VCH}

25563; d) Z. Chen, Z. Wang, X. Li, Y. Lin, N. Luo, M. Long, N. Zhao, J.-B. Xu, ACS Nano 2017, 11, 4507.

[39] a) H. Y. Jeong, D.-S. Lee, H. K. Choi, D. H. Lee, J.-E. Kim, J. Y. Lee, W. J. Lee, S. O. Kim, S.-Y. Choi, Appl. Phys. Lett. 2010, 96, 213105; b) X. Dong, J. Chen, Y. Ma, J. Wang, M. B. Chan-Park, X. Liu, L. Wang, W. Huang, P. Chen, Chem. Commun. (Cambridge, U. K.) 2012, 48, 10660.

[40] Y. Han, Y. Jiang, C. Gao, ACS Appl. Mater. Interfaces 2015, 7, 8147.

[41] T. V. Cuong, V. H. Pham, J. S. Chung, E. W. Shin, D. H. Yoo, S. H. Hahn, J. S. Huh, G. H. Rue, E. J. Kim, S. H. Hur, P. A. Kohl, Mater. Lett. 2010, 64, 2479.

[42] a) J. Yi, J. M. Lee, W. I. Park, Sens. Actuator B-Chem. 2011, 155, 264; b) S. J. Lee, Y. B. Lee, Y. R. Lim, J. K. Han, I. S. Jeon, G. Bae, Y. Yoon, W. Song, S. Myung, J. Lim, K. S. An, S. S. Lee, Sci. Rep. 2019, 9, 9376; c) Y. Huang, Z. Huang, Z. Zhong, X. Yang, Q. Hong, H. Wang, S. Huang, N. Gao, X. Chen, D. Cai, J. Kang, Sci. Rep. 2018, 8, 13721.

[43] a) A. G. Ricciardulli, S. Yang, G.-J. A. H. Wetzelaer, X. Feng, P. W. M. Blom, Adv. Funct. Mater. 2018, 28, 1706010; b) H. Dong, Z. Wu, Y. Jiang, W. Liu, X. Li, B. Jiao, W. Abbas, X. Hou, ACS Appl. Mater. Interfaces 2016, 8, 31212.

[44] P. Z. Sun, Q. Yang, W. J. Kuang, Y. V. Stebunov, W. Q. Xiong, J. Yu, R. R. Nair, M. I. Katsnelson, S. J. Yuan, I. V. Grigorieva, M. Lozada-Hidalgo, F. C. Wang, A. K. Geim, Nature 2020, 579, 229.

[45] W. K. Chee, H. N. Lim, Z. Zainal, N. M. Huang, I. Harrison, Y. Andou, J. Phys. Chem. C 2016, 120, 4153.

[46] P. Solís-Fernández, M. Bissett, H. Ago, Chem. Soc. Rev. 2017, 46, 4572.

[47] a) H. Wang, F. Liu, W. Fu, Z. Fang, W. Zhou, Z. Liu, Nanoscale 2014, 6, 12250; b) A. K. Geim, I. V. Grigorieva, Nature 2013, 499, 419.

[48] C. R. Dean, A. F. Young, I. Meric, C. Lee, L. Wang, S. Sorgenfrei, K. Watanabe, T. Taniguchi, P. Kim, K. L. Shepard, J. Hone, Nat. Nanotechnol. 2010, 5, 722.

[49] a) K. S. Novoselov, A. Mishchenko, A. Carvalho, A. H. Castro Neto, Science 2016, 353, aac9439; b) Q. Li, M. Liu, Y. Zhang, Z. Liu, Small 2016, 12, 32; c) M. Kan, Y. Li, Q. Sun, Wiley Interdiscip. Rev. Comput. Mol. Sci. 2016, 6, 65; d) M. Yankowitz, Q. Ma, P. Jarillo-Herrero, B. J. LeRoy, Nat. Rev. Phys. 2019, 1, 112. 


\section{WILEY-VCH}

[50] C. R. Dean, L. Wang, P. Maher, C. Forsythe, F. Ghahari, Y. Gao, J. Katoch, M. Ishigami, P. Moon, M. Koshino, T. Taniguchi, K. Watanabe, K. L. Shepard, J. Hone, P. Kim, Nature 2013, 497, 598.

[51] M. Yankowitz, J. M. Xue, D. Cormode, J. D. Sanchez-Yamagishi, K. Watanabe, T. Taniguchi, P. Jarillo-Herrero, P. Jacquod, B. J. LeRoy, Nat. Phys. 2012, 8, 382.

[52] J. Jung, A. M. DaSilva, A. H. MacDonald, S. Adam, Nat Commun 2015, 6, 6308.

[53] L. Britnell, R. V. Gorbachev, R. Jalil, B. D. Belle, F. Schedin, A. Mishchenko, T. Georgiou, M. I. Katsnelson, L. Eaves, S. V. Morozov, N. M. R. Peres, J. Leist, A. K. Geim, K. S. Novoselov, L. A. Ponomarenko, Science 2012, 335, 947.

[54] S. J. Haigh, A. Gholinia, R. Jalil, S. Romani, L. Britnell, D. C. Elias, K. S. Novoselov, L. A. Ponomarenko, A. K. Geim, R. Gorbachev, Nat. Mater. 2012, 11, 764.

[55] a) L. Wang, I. Meric, P. Y. Huang, Q. Gao, Y. Gao, H. Tran, T. Taniguchi, K. Watanabe, L. M. Campos, D. A. Muller, J. Guo, P. Kim, J. Hone, K. L. Shepard, C. R. Dean, Science 2013, 342, 614; b) P. J. Zomer, S. P. Dash, N. Tombros, B. J. van Wees, Appl. Phys. Lett. 2011, 99, 232104.

[56] a) G. Gao, W. Gao, E. Cannuccia, J. Taha-Tijerina, L. Balicas, A. Mathkar, T. N. Narayanan, Z. Liu, B. K. Gupta, J. Peng, Y. Yin, A. Rubio, P. M. Ajayan, Nano Lett. 2012, 12, 3518; b) J. Sun, H.-W. Lee, M. Pasta, H. Yuan, G. Zheng, Y. Sun, Y. Li, Y. Cui, Nat. Nanotechnol. 2015, 10, 980; c) S. Sinha, Jyotsna, S. K. Arora, Mater TodayProc 2020, 21, 1840.

[57] Z. Liu, L. Song, S. Zhao, J. Huang, L. Ma, J. Zhang, J. Lou, P. M. Ajayan, Nano Lett. 2011, 11, 2032.

[58] X. Ding, G. Ding, X. Xie, F. Huang, M. Jiang, Carbon 2011, 49, 2522.

[59] Z. Yan, Z. Peng, Z. Sun, J. Yao, Y. Zhu, Z. Liu, P. M. Ajayan, J. M. Tour, ACS Nano 2011, 5, 8187 .

[60] X. Song, T. Gao, Y. Nie, J. Zhuang, J. Sun, D. Ma, J. Shi, Y. Lin, F. Ding, Y. Zhang, Z. Liu, Nano Lett. 2016, 16, 6109.

[61] X. Ling, Y.-H. Lee, Y. Lin, W. Fang, L. Yu, M. S. Dresselhaus, J. Kong, Nano Lett. 2014, 14, 464 .

[62] K. Roy, M. Padmanabhan, S. Goswami, T. P. Sai, G. Ramalingam, S. Raghavan, A. Ghosh, Nat. Nanotechnol. 2013, 8, 826. 


\section{WILEY-VCH}

[63] Y. Tsuboi, F. Wang, D. Kozawa, K. Funahashi, S. Mouri, Y. Miyauchi, T. Takenobu, K. Matsuda, Nanoscale 2015, 7, 14476.

[64] a) S. Bertolazzi, D. Krasnozhon, A. Kis, ACS Nano 2013, 7, 3246; b) S. Bertolazzi, P. Bondavalli, S. Roche, T. San, S. Y. Choi, L. Colombo, F. Bonaccorso, P. Samori, Adv. Mater. 2019, 31, e1806663.

[65] F. Withers, O. Del Pozo-Zamudio, A. Mishchenko, A. P. Rooney, A. Gholinia, K. Watanabe, T. Taniguchi, S. J. Haigh, A. K. Geim, A. I. Tartakovskii, K. S. Novoselov, Nat. Mater. 2015, 14, 301.

[66] a) L. Peng, Y. Zhu, H. Li, G. Yu, Small 2016, 12, 6183; b) E. Pomerantseva, Y. Gogotsi, Nat. Energy 2017, 2, 17089.

[67] M. A. Bissett, I. A. Kinloch, R. A. W. Dryfe, ACS Appl. Mater. Interfaces 2015, 7, 17388 .

[68] a) W. S. V. Lee, E. Peng, T. A. J. Loh, X. Huang, J. M. Xue, Nanoscale 2016, 8, 8042;

b) Y. Teng, H. Zhao, Z. Zhang, Z. Li, Q. Xia, Y. Zhang, L. Zhao, X. Du, Z. Du, P. Lv, K. Świerczek, ACS Nano 2016, 10, 8526.

[69] L. Ci, L. Song, C. Jin, D. Jariwala, D. Wu, Y. Li, A. Srivastava, Z. F. Wang, K. Storr, L. Balicas, F. Liu, P. M. Ajayan, Nat. Mater. 2010, 9, 430.

[70] a) Z. Liu, L. Ma, G. Shi, W. Zhou, Y. Gong, S. Lei, X. Yang, J. Zhang, J. Yu, K. P. Hackenberg, A. Babakhani, J.-C. Idrobo, R. Vajtai, J. Lou, P. M. Ajayan, Nat. Nanotechnol. 2013, 8, 119; b) Y. Gong, G. Shi, Z. Zhang, W. Zhou, J. Jung, W. Gao, L. Ma, Y. Yang, S. Yang, G. You, R. Vajtai, Q. Xu, A. H. MacDonald, B. I. Yakobson, J. Lou, Z. Liu, P. M. Ajayan, Nat. Comm. 2014, 5, 3193.

[71] a) X. Ling, Y. Lin, Q. Ma, Z. Wang, Y. Song, L. Yu, S. Huang, W. Fang, X. Zhang, A. L. Hsu, Y. Bie, Y.-H. Lee, Y. Zhu, L. Wu, J. Li, P. Jarillo-Herrero, M. Dresselhaus, T. Palacios, J. Kong, Adv. Mater. 2016, 28, 2322; b) M. Zhao, Y. Ye, Y. Han, Y. Xia, H. Zhu, S. Wang, Y. Wang, D. A. Muller, X. Zhang, Nat. Nanotechnol. 2016, 11, 954; c) M. H. D. Guimarães, H. Gao, Y. Han, K. Kang, S. Xie, C.-J. Kim, D. A. Muller, D. C. Ralph, J. Park, ACS Nano 2016, 10, 6392.

[72] E. Bekyarova, M. E. Itkis, P. Ramesh, C. Berger, M. Sprinkle, W. A. de Heer, R. C. Haddon, J. Am. Chem. Soc. 2009, 131, 1336.

[73] L.-H. Liu, M. Yan, J. Mater. Chem. 2011, 21, 3273. 


\section{WILEY-VCH}

[74] V. Georgakilas, M. Otyepka, A. B. Bourlinos, V. Chandra, N. Kim, K. C. Kemp, P. Hobza, R. Zboril, K. S. Kim, Chem. Rev. 2012, 112, 6156.

[75] A. Sinitskii, A. Dimiev, D. A. Corley, A. A. Fursina, D. V. Kosynkin, J. M. Tour, ACS Nano 2010, 4, 1949.

[76] S. Niyogi, E. Bekyarova, M. E. Itkis, H. Zhang, K. Shepperd, J. Hicks, M. Sprinkle, C. Berger, C. N. Lau, W. A. Deheer, E. H. Conrad, R. C. Haddon, Nano Lett. 2010, 10, 4061.

[77] H. T. Liu, S. M. Ryu, Z. Y. Chen, M. L. Steigerwald, C. Nuckolls, L. E. Brus, J. Am. Chem. Soc. 2009, 131, 17099.

[78] a) V. Georgakilas, A. B. Bourlinos, R. Zboril, T. A. Steriotis, P. Dallas, A. K. Stubos, C. Trapalis, Chem. Commun. (Cambridge, U. K.) 2010, 46, 1766; b) X. Zhang, L. Hou, A. Cnossen, A. C. Coleman, O. Ivashenko, P. Rudolf, B. J. van Wees, W. R. Browne, B. L. Feringa, Chem. - Eur. J. 2011, 17, 8957.

[79] S. Vadukumpully, J. Gupta, Y. P. Zhang, G. Q. Xu, S. Valiyaveettil, Nanoscale 2011, 3,303 .

[80] T. A. Strom, E. P. Dillon, C. E. Hamilton, A. R. Barron, Chem. Commun. (Cambridge, U. K.) 2010, 46, 4097.

[81] X. Zhong, J. Jin, S. W. Li, Z. Y. Niu, W. Q. Hu, R. Li, J. T. Ma, Chem. Commun. (Cambridge, U. K.) 2010, 46, 7340.

[82] C. Gómez-Navarro, R. T. Weitz, A. M. Bittner, M. Scolari, A. Mews, M. Burghard, K. Kern, Nano Lett. 2007, 7, 3499.

[83] Y. Liu, J. Zhou, X. Zhang, Z. Liu, X. Wan, J. Tian, T. Wang, Y. Chen, Carbon 2009, 47,3113 .

[84] D. Yu, Y. Yang, M. Durstock, J.-B. Baek, L. Dai, ACS Nano 2010, 4, 5633.

[85] Y. F. Xu, Z. B. Liu, X. L. Zhang, Y. Wang, J. G. Tian, Y. Huang, Y. F. Ma, X. Y. Zhang, Y. S. Chen, Adv. Mater. 2009, 21, 1275.

[86] J. Shen, M. Shi, B. Yan, H. Ma, N. Li, Y. Hu, M. Ye, Colloids Surf., B 2010, 81, 434.

[87] a) F. Liu, J. Y. Choi, T. S. Seo, Biosens. Bioelectron. 2010, 25, 2361; b) N. Mohanty, V. Berry, Nano Lett. 2008, 8, 4469.

[88] Z. Liu, J. T. Robinson, X. M. Sun, H. J. Dai, J. Am. Chem. Soc. 2008, 130, 10876.

[89] H. J. Salavagione, M. A. Gomez, G. Martinez, Macromolecules 2009, 42, 6331. 


\section{WILEY-VCH}

[90] a) O. C. Compton, D. A. Dikin, K. W. Putz, L. C. Brinson, S. T. Nguyen, Adv. Mater. 2010, 22, 892; b) H. R. Thomas, A. J. Marsden, M. Walker, N. R. Wilson, J. P. Rourke, Encyclopedia of imaging science and technology 2014, 53, 7613; c) S. Chakraborty, S. Saha, V. R. Dhanak, K. Biswas, M. Barbezat, G. P. Terrasi, A. K. Chakraborty, RSC $A d v$. 2016, 6, 67916; d) I. A. Vacchi, S. Guo, J. Raya, A. Bianco, C. Ménard-Moyon, Chem. - Eur. J. 2020, 26, 6591; e) S. Guo, Y. Nishina, A. Bianco, C. Ménard-Moyon, Angew. Chem., Int. Ed. 2020, 59, 1542.

[91] I. A. Vacchi, C. Spinato, J. Raya, A. Bianco, C. Ménard-Moyon, Nanoscale 2016, 8, 13714 .

[92] C. Shan, H. Yang, D. Han, Q. Zhang, A. Ivaska, L. Niu, Langmuir 2009, 25, 12030.

[93] a) W. Yuan, A. Liu, L. Huang, C. Li, G. Shi, Adv. Mater. 2013, 25, 766; b) N. Hu, Y. Wang, J. Chai, R. Gao, Z. Yang, E. S.-W. Kong, Y. Zhang, Sens. Actuator B-Chem. 2012, 163, 107; c) N. L. Teradal, S. Marx, A. Morag, R. Jelinek, J. Mater. Chem. C 2017, 5, 1128.

[94] a) R. Seenivasan, W.-J. Chang, S. Gunasekaran, ACS Appl. Mater. Interfaces 2015, 7, 15935; b) C. J. Madadrang, H. Y. Kim, G. Gao, N. Wang, J. Zhu, H. Feng, M. Gorring, M. L. Kasner, S. Hou, ACS Appl. Mater. Interfaces 2012, 4, 1186.

[95] S. H. Lee, D. R. Dreyer, J. An, A. Velamakanni, R. D. Piner, S. Park, Y. Zhu, S. O. Kim, C. W. Bielawski, R. S. Ruoff, Macromol. Rapid Commun. 2010, 31, 281.

[96] a) B. Das, K. E. Prasad, U. Ramamurty, C. N. R. Rao, Nanotechnology 2009, 20; b) M. Fang, K. G. Wang, H. B. Lu, Y. L. Yang, S. Nutt, J. Mater. Chem. 2009, 19, 7098; c) T. Ramanathan, A. A. Abdala, S. Stankovich, D. A. Dikin, M. Herrera-Alonso, R. D. Piner, D. H. Adamson, H. C. Schniepp, X. Chen, R. S. Ruoff, S. T. Nguyen, I. A. Aksay, R. K. Prud'homme, L. C. Brinson, Nat. Nanotechnol. 2008, 3, 327; d) G. Gonçalves, P. A. A. P. Marques, A. Barros-Timmons, I. Bdkin, M. K. Singh, N. Emami, J. Grácio, J. Mater. Chem. 2010, 20, 9927; e) K. P. Pramoda, H. Hussain, H. M. Koh, H. R. Tan, C. B. He, J. Polym. Sci., Part A: Polym. Chem. 2010, 48, 4262.

[97] Y. Yang, Y. Xie, L. Pang, M. Li, X. Song, J. Wen, H. Zhao, Langmuir 2013, 29, 10727.

[98] a) Y. Li, M. Zhou, Y. Wang, Q. Pan, Q. Gong, Z. Xia, Y. Li, Carbon 2019, 147, 519; b) R. Li, Y. Yang, D. Wu, K. Li, Y. Qin, Y. Tao, Y. Kong, Chem. Commun. (Cambridge, U. K.) 2019, 55, 1738; c) S. K. Yadav, R. Kumar, A. K. Sundramoorthy, R. K. Singh, 


\section{WILEY-VCH}

C. M. Koo, $R S C A d v$. 2016, 6, 52945; d) Z.-F. Li, H. Zhang, Q. Liu, Y. Liu, L. Stanciu, J. Xie, Carbon 2014, 71, 257.

[99] J. Lehn, Science 1993, 260, 1762.

[100] a) X.-F. Zhang, X. Shao, J. Photochem. Photobiol., A 2014, 278, 69; b) J. Wang, Z. Chen, B. Chen, Environ. Sci. Technol. 2014, 48, 4817.

[101] X. Chen, K. Shehzad, L. Gao, M. Long, H. Guo, S. Qin, X. Wang, F. Wang, Y. Shi, W. Hu, Y. Xu, X. Wang, Adv. Mater. 2019, 32, 1902039.

[102] Q. Su, S. Pang, V. Alijani, C. Li, X. Feng, K. Müllen, Adv. Mater. 2009, 21, 3191.

[103] A. Ciesielski, P. Samorì, Adv. Mater. 2016, 28, 6030.

[104] A. Schlierf, H. Yang, E. Gebremedhn, E. Treossi, L. Ortolani, L. Chen, A. Minoia, V. Morandi, P. Samorì, C. Casiraghi, D. Beljonne, V. Palermo, Nanoscale 2013, 5, 4205.

[105] Z. Chen, Y. Wang, Y. Shang, A. Umar, P. Xie, Q. Qi, G. Zhou, Sci. Rep. 2017, 7, 2713.

[106] S. Qu, M. Li, L. Xie, X. Huang, J. Yang, N. Wang, S. Yang, ACS Nano 2013, 7, 4070.

[107] a) X. Tian, J. Xu, X. Wang, J. Phys. Chem. B 2010, 114, 11377; b) S. M. Kozlov, F. Viñes, A. Görling, Adv. Mater. 2011, 23, 2638.

[108] W. Zhang, C.-T. Lin, K.-K. Liu, T. Tite, C.-Y. Su, C.-H. Chang, Y.-H. Lee, C.-W. Chu, K.-H. Wei, J.-L. Kuo, L.-J. Li, ACS Nano 2011, 5, 7517.

[109] W. Chen, S. Chen, D. C. Qi, X. Y. Gao, A. T. S. Wee, J. Am. Chem. Soc. 2007, 129, 10418.

[110] J. Mao, H. Zhang, Y. Jiang, Y. Pan, M. Gao, W. Xiao, H. J. Gao, J. Am. Chem. Soc. 2009, 131, 14136.

[111] S. K. Hämäläinen, M. Stepanova, R. Drost, P. Liljeroth, J. Lahtinen, J. Sainio, J. Phys. Chem. C 2012, 116, 20433.

[112] Y. Xu, L. Zhao, H. Bai, W. Hong, C. Li, G. Shi, J. Am. Chem. Soc. 2009, 131, 13490.

[113] B.-P. Jiang, L.-F. Hu, D.-J. Wang, S.-C. Ji, X.-C. Shen, H. Liang, J. Mater. Chem. B 2014, 2, 7141 .

[114] Y. Guo, L. Deng, J. Li, S. Guo, E. Wang, S. Dong, ACS Nano 2011, 5, 1282.

[115] a) S. Haar, A. Ciesielski, J. Clough, H. Yang, R. Mazzaro, F. Richard, S. Conti, N. Merstorf, M. Cecchini, V. Morandi, C. Casiraghi, P. Samorì, Small 2015, 11, 1691; b) A. Ciesielski, S. Haar, M. El Gemayel, H. Yang, J. Clough, G. Melinte, M. Gobbi, E. Orgiu, M. V. Nardi, G. Ligorio, V. Palermo, N. Koch, O. Ersen, C. Casiraghi, P. Samorì, Angew. Chem., Int. Ed. 2014, 53, 10355. 


\section{WILEY-VCH}

[116] B. Li, A. V. Klekachev, M. Cantoro, C. Huyghebaert, A. Stesmans, I. Asselberghs, S. De Gendt, S. De Feyter, Nanoscale 2013, 5, 9640.

[117] R. Phillipson, C. J. Lockhart de la Rosa, J. Teyssandier, P. Walke, D. Waghray, Y. Fujita, J. Adisoejoso, K. S. Mali, I. Asselberghs, C. Huyghebaert, H. Uji-i, S. De Gendt, S. De Feyter, Nanoscale 2016, 8, 20017.

[118] a) M. Gobbi, S. Bonacchi, J. X. Lian, Y. Liu, X.-Y. Wang, M.-A. Stoeckel, M. A. Squillaci, G. D’Avino, A. Narita, K. Müllen, X. Feng, Y. Olivier, D. Beljonne, P. Samorì, E. Orgiu, Nat. Comm. 2017, 8, 14767; b) M. Gobbi, S. Bonacchi, J. X. Lian, A. Vercouter, S. Bertolazzi, B. Zyska, M. Timpel, R. Tatti, Y. Olivier, S. Hecht, M. V. Nardi, D. Beljonne, E. Orgiu, P. Samorì, Nat. Comm. 2018, 9, 2661; c) M. Gobbi, A. Galanti, M.-A. Stoeckel, B. Zyska, S. Bonacchi, S. Hecht, P. Samorì, Nat. Comm. 2020, 11,4731 .

[119] T. Premkumar, K. E. Geckeler, Prog. Polym. Sci. 2012, 37, 515.

[120] B. G. Choi, H. Park, T. J. Park, M. H. Yang, J. S. Kim, S.-Y. Jang, N. S. Heo, S. Y. Lee, J. Kong, W. H. Hong, ACS Nano 2010, 4, 2910.

[121] H. Bai, Y. Xu, L. Zhao, C. Li, G. Shi, Chem. Commun. (Cambridge, U. K.) 2009, 13, 1667.

[122] D.-W. Wang, F. Li, J. Zhao, W. Ren, Z.-G. Chen, J. Tan, Z.-S. Wu, I. Gentle, G. Q. Lu, H.-M. Cheng, ACS Nano 2009, 3, 1745.

[123] A. J. Patil, J. L. Vickery, T. B. Scott, S. Mann, Adv. Mater. 2009, 21, 3159.

[124] J. Liu, Y. Li, Y. Li, J. Li, Z. Deng, J. Mater. Chem. 2010, 20, 900.

[125] Y. Xu, Q. Wu, Y. Sun, H. Bai, G. Shi, ACS Nano 2010, 4, 7358.

[126] Y. Liang, D. Wu, X. Feng, K. Müllen, Adv. Mater. 2009, 21, 1679.

[127] X. Zhao, Q. Zhang, Y. Hao, Y. Li, Y. Fang, D. Chen, Macromolecules 2010, 43, 9411.

[128] M. Hu, B. Mi, J. Membr. Sci. 2014, 469, 80.

[129] J. Zou, F. Kim, Nat. Comm. 2014, 5, 5254.

[130] D. Zhang, J. Tong, B. Xia, Sens. Actuator B-Chem. 2014, 197, 66.

[131] S. Dervin, D. D. Dionysiou, S. C. Pillai, Nanoscale 2016, 8, 15115.

[132] B. F. Machado, P. Serp, Catal. Sci. Technol. 2012, 2, 54.

[133] a) Y. Zhang, T. R. Nayak, H. Hong, W. Cai, Nanoscale 2012, 4, 3833; b) S. Syama, P. V. Mohanan, Nano-Micro Lett. 2019, 11. 


\section{WILEY-VCH}

[134] a) E. Yoo, J. Kim, E. Hosono, H.-s. Zhou, T. Kudo, I. Honma, Nano Lett. 2008, 8, 2277;

b) S.-M. Paek, E. Yoo, I. Honma, Nano Lett. 2009, 9, 72; c) X. Zhou, L.-J. Wan, Y.-G. Guo, Adv. Mater. 2013, 25, 2152.

[135] a) W. J. Yu, Y. Liu, H. Zhou, A. Yin, Z. Li, Y. Huang, X. Duan, Nat. Nanotechnol. 2013, 8, 952; b) G. Konstantatos, M. Badioli, L. Gaudreau, J. Osmond, M. Bernechea, F. P. G. de Arquer, F. Gatti, F. H. L. Koppens, Nat. Nanotechnol. 2012, 7, 363; c) S. J. Kim, W. Song, S. Kim, M.-A. Kang, S. Myung, S. Sook Lee, J. Lim, K.-S. An, Nanotechnology 2016, 27, 075709.

[136] A. Morelos-Gomez, R. Cruz-Silva, H. Muramatsu, J. Ortiz-Medina, T. Araki, T. Fukuyo, S. Tejima, K. Takeuchi, T. Hayashi, M. Terrones, M. Endo, Nat. Nanotechnol. 2017, 12, 1083.

[137] D. Cohen-Tanugi, J. C. Grossman, Nano Lett. 2012, 12, 3602.

[138] J. Liu, S. Li, B. Zhang, Y. Wang, Y. Gao, X. Liang, Y. Wang, G. Lu, J. Colloid Interface Sci. 2017, 504, 206.

[139] L. N. Suvarapu, Y.-K. Seo, S.-O. Baek, Rev. Anal. Chem. 2013, 32.

[140] J. H. An, S. J. Park, O. S. Kwon, J. Bae, J. Jang, ACS Nano 2013, 7, 10563.

[141] S. Shiva Kumar, V. Himabindu, Materials Science for Energy Technologies 2019, 2, 442.

[142] H. Zhang, X. Yu, D. Guo, B. Qu, M. Zhang, Q. Li, T. Wang, ACS Appl. Mater. Interfaces 2013, 5, 7335 .

[143] F. Schedin, A. K. Geim, S. V. Morozov, E. W. Hill, P. Blake, M. I. Katsnelson, K. S. Novoselov, Nat. Mater. 2007, 6, 652.

[144] C. Wang, L. Yin, L. Zhang, D. Xiang, R. Gao, Sensors 2010, 10, 2088.

[145] W. Li, X. Geng, Y. Guo, J. Rong, Y. Gong, L. Wu, X. Zhang, P. Li, J. Xu, G. Cheng, M. Sun, L. Liu, ACS Nano 2011, 5, 6955.

[146] L. Huang, Z. Wang, J. Zhang, J. Pu, Y. Lin, S. Xu, L. Shen, Q. Chen, W. Shi, ACS Appl. Mater. Interfaces 2014, 6, 7426.

[147] a) S. Deng, V. Tjoa, H. M. Fan, H. R. Tan, D. C. Sayle, M. Olivo, S. Mhaisalkar, J. Wei, C. H. Sow, J. Am. Chem. Soc. 2012, 134, 4905; b) W. Yang, P. Wan, X. Zhou, J. Hu, Y. Guan, L. Feng, ACS Appl. Mater. Interfaces 2014, 6, 21093. 


\section{WILEY-VCH}

[148] L. Al-Mashat, K. Shin, K. Kalantar-zadeh, J. D. Plessis, S. H. Han, R. W. Kojima, R. B. Kaner, D. Li, X. Gou, S. J. Ippolito, W. Wlodarski, J. Phys. Chem. C 2010, 114, 16168.

[149] X. Huang, N. Hu, R. Gao, Y. Yu, Y. Wang, Z. Yang, E. Siu-Wai Kong, H. Wei, Y. Zhang, J. Mater. Chem. 2012, 22, 22488.

[150] H. Bai, K. Sheng, P. Zhang, C. Li, G. Shi, J. Mater. Chem. 2011, 21, 18653.

[151] C. Anichini, A. Aliprandi, S. M. Gali, F. Liscio, V. Morandi, A. Minoia, D. Beljonne, A. Ciesielski, P. Samorì, ACS Appl. Mater. Interfaces 2020, 12, 44017.

[152] R. Furlan de Oliveira, P. A. Livio, V. Montes-García, S. Ippolito, M. Eredia, P. FanjulBolado, M. B. González García, S. Casalini, P. Samorì, Adv. Funct. Mater. 2019, 29, 1905375.

[153] T. Zhang, Z. Cheng, Y. Wang, Z. Li, C. Wang, Y. Li, Y. Fang, Nano Lett. 2010, 10, 4738 .

[154] H. G. Sudibya, Q. He, H. Zhang, P. Chen, ACS Nano 2011, 5, 1990.

[155] M. Li, X. Zhou, W. Ding, S. Guo, N. Wu, Biosens. Bioelectron. 2013, 41, 889.

[156] Y. Wen, F. Xing, S. He, S. Song, L. Wang, Y. Long, D. Li, C. Fan, Chem. Commun. (Cambridge, U. K.) 2010, 46, 2596.

[157] W. Fu, L. Feng, G. Panaitov, D. Kireev, D. Mayer, A. Offenhäusser, H.-J. Krause, Sci. Adv. 2017, 3, e1701247.

[158] S. Guo, D. Wen, Y. Zhai, S. Dong, E. Wang, ACS Nano 2010, 4, 3959.

[159] J. Luo, S. Jiang, H. Zhang, J. Jiang, X. Liu, Anal. Chim. Acta 2012, 709, 47.

[160] C.-B. Huang, S. Witomska, A. Aliprandi, M.-A. Stoeckel, M. Bonini, A. Ciesielski, P. Samorì, Adv. Mater. 2019, 31, 1804600.

[161] S. Liu, X. Wu, D. Zhang, C. Guo, P. Wang, W. Hu, X. Li, X. Zhou, H. Xu, C. Luo, J. Zhang, J. Chu, ACS Appl. Mater. Interfaces 2017, 9, 24148.

[162] Q. Li, Z. Ullah, W. Li, Y. Guo, J. Xu, R. Wang, Q. Zeng, M. Chen, C. Liu, L. Liu, Small 2016, 12, 5058.

[163] J. S. Bunch, S. S. Verbridge, J. S. Alden, A. M. van der Zande, J. M. Parpia, H. G. Craighead, P. L. McEuen, Nano Lett. 2008, 8, 2458.

[164] M. E. Suk, N. R. Aluru, J. Phys. Chem. Lett. 2010, 1, 1590.

[165] S. P. Surwade, S. N. Smirnov, I. V. Vlassiouk, R. R. Unocic, G. M. Veith, S. Dai, S. M. Mahurin, Nat. Nanotechnol. 2015, 10, 459. 


\section{WILEY-VCH}

[166] a) A. Nicolaï, B. G. Sumpter, V. Meunier, Phys. Chem. Chem. Phys. 2014, 16, 8646; b) R. R. Nair, H. A. Wu, P. N. Jayaram, I. V. Grigorieva, A. K. Geim, Science 2012, 335, 442.

[167] D. Pakulski, W. Czepa, S. D. Buffa, A. Ciesielski, P. Samorì, Adv. Funct. Mater. 2019, 30, 1902394.

[168] F. Perreault, A. Fonseca de Faria, M. Elimelech, Chem. Soc. Rev. 2015, 44, 5861.

[169] M. Hu, B. Mi, Environ. Sci. Technol. 2013, 47, 3715.

[170] J.-Q. Hu, Z. Liu, Z.-H. Chen, Q.-W. Cai, X.-Y. Li, R. Xie, X.-J. Ju, W. Wang, L.-Y. Chu, Ind. Eng. Chem. Res. 2020, 59, 12441.

[171] S. J. Gao, H. Qin, P. Liu, J. Jin, J. Mater. Chem. A 2015, 3, 6649.

[172] A. Hassanpour, S. Nahar, X. Tong, G. Zhang, M. A. Gauthier, S. Sun, Desalination 2020, 475, 114174.

[173] T. Pradeep, Anshup, Thin Solid Films 2009, 517, 6441.

[174] a) K. Zhang, V. Dwivedi, C. Chi, J. Wu, J. Hazard. Mater. 2010, 182, 162; b) X. Luo, C. Wang, S. Luo, R. Dong, X. Tu, G. Zeng, Chem. Eng. J. (Lausanne) 2012, 187, 45.

[175] a) X. Yang, C. Chen, J. Li, G. Zhao, X. Ren, X. Wang, RSC Adv. 2012, 2, 8821; b) X. Guo, B. Du, Q. Wei, J. Yang, L. Hu, L. Yan, W. Xu, J. Hazard. Mater. 2014, 278, 211.

[176] H. Jabeen, V. Chandra, S. Jung, J. W. Lee, K. S. Kim, S. B. Kim, Nanoscale 2011, 3, 3583 .

[177] V. Chandra, K. S. Kim, Chem. Commun. (Cambridge, U. K.) 2011, 47, 3942.

[178] A. Docoslis, S. Li, X. Lu, Y. Xue, J. Lei, T. Zheng, C. Wang, PLoS One 2012, 7, e43328.

[179] L. Fan, C. Luo, M. Sun, H. Qiu, X. Li, Colloids Surf., B 2013, 103, 601.

[180] N. Zhang, H. Qiu, Y. Si, W. Wang, J. Gao, Carbon 2011, 49, 827.

[181] A. Shahzad, W. Miran, K. Rasool, M. Nawaz, J. Jang, S.-R. Lim, D. S. Lee, RSC Adv. 2017, 7, 9764.

[182] L. Fan, C. Luo, X. Li, F. Lu, H. Qiu, M. Sun, J. Hazard. Mater. 2012, 215-216, 272.

[183] T.-D. Nguyen-Phan, V. H. Pham, E. J. Kim, E.-S. Oh, S. H. Hur, J. S. Chung, B. Lee, E. W. Shin, Appl. Surf. Sci. 2012, 258, 4551.

[184] B. Li, H. Cao, G. Yin, J. Mater. Chem. 2011, 21, 13765.

[185] B. Xia, Y. Yan, X. Wang, X. W. Lou, Mater. Horiz. 2014, 1, 379.

[186] X. Li, L. Zhi, Chem. Soc. Rev. 2018, 47, 3189.

[187] Z. Yang, H. Nie, X. a. Chen, X. Chen, S. Huang, J. Power Sources 2013, 236, 238. 


\section{WILEY-VCH}

[188] S. Liu, C. Liu, W. Wang, B. Cheng, J. Yu, Nanoscale 2012, 4, 3193.

[189] X.-Y. Zhang, H.-P. Li, X.-L. Cui, Y. Lin, J. Mater. Chem. 2010, 20, 2801.

[190] a) Q. Xiang, J. Yu, M. Jaroniec, Nanoscale 2011, 3, 3670; b) H. Zhang, X. Lv, Y. Li, Y. Wang, J. Li, ACS Nano 2009, 4, 380; c) J. S. Lee, K. H. You, C. B. Park, Adv. Mater. 2012, 24, 1084.

[191] L.-L. Tan, S.-P. Chai, A. R. Mohamed, ChemSusChem 2012, 5, 1868.

[192] G. Williams, B. Seger, P. V. Kamat, ACS Nano 2008, 2, 1487.

[193] a) C. Chen, W. Cai, M. Long, B. Zhou, Y. Wu, D. Wu, Y. Feng, ACS Nano 2010, 4, 6425; b) J. Liu, H. Bai, Y. Wang, Z. Liu, X. Zhang, D. D. Sun, Adv. Funct. Mater. 2010, 20, 4175 .

[194] Y.-P. Xiao, S. Wan, X. Zhang, J.-S. Hu, Z.-D. Wei, L.-J. Wan, Chem. Commun. (Cambridge, U. K.) 2012, 48, 10331.

[195] a) J. N. Tiwari, K. C. Kemp, K. Nath, R. N. Tiwari, H.-G. Nam, K. S. Kim, ACS Nano 2013, 7, 9223; b) J. N. Tiwari, K. Nath, S. Kumar, R. N. Tiwari, K. C. Kemp, N. H. Le, D. H. Youn, J. S. Lee, K. S. Kim, Nat. Comm. 2013, 4, 2221.

[196] Y. Liang, Y. Li, H. Wang, J. Zhou, J. Wang, T. Regier, H. Dai, Nat. Mater. 2011, 10, 780.

[197] Y. Li, H. Wang, L. Xie, Y. Liang, G. Hong, H. Dai, J. Am. Chem. Soc. 2011, 133, 7296.

[198] X. Zheng, J. Xu, K. Yan, H. Wang, Z. Wang, S. Yang, Chem. Mater. 2014, 26, 2344.

[199] R. K. Biroju, D. Das, R. Sharma, S. Pal, L. P. L. Mawlong, K. Bhorkar, P. K. Giri, A. K. Singh, T. N. Narayanan, ACS Energy Lett. 2017, 2, 1355.

[200] a) A. Ruiz, M. A. Lucherelli, D. Murera, D. Lamon, C. Ménard-Moyon, A. Bianco, Carbon 2020, 170, 347; b) A. Bianco, Angew. Chem., Int. Ed. 2013, 52, 4986.

[201] X. Sun, Z. Liu, K. Welsher, J. T. Robinson, A. Goodwin, S. Zaric, H. Dai, Nano Res. 2008, 1, 203.

[202] Z. Liu, J. T. Robinson, X. Sun, H. Dai, J. Am. Chem. Soc. 2008, 130, 10876.

[203] C. Wang, J. Li, C. Amatore, Y. Chen, H. Jiang, X.-M. Wang, Angew. Chem., Int. Ed. 2011, 50, 11644.

[204] M. de Sousa, L. A. Visani de Luna, L. C. Fonseca, S. Giorgio, O. L. Alves, ACS Appl. Nano Mater. 2018, 1, 922.

[205] G. Reina, J. M. González-Domínguez, A. Criado, E. Vázquez, A. Bianco, M. Prato, Chem. Soc. Rev. 2017, 46, 4400. 


\section{WILEY-VCH}

[206] K. Yang, S. Zhang, G. Zhang, X. Sun, S.-T. Lee, Z. Liu, Nano Lett. 2010, 10, 3318.

[207] P. Rong, K. Yang, A. Srivastan, D. O. Kiesewetter, X. Yue, F. Wang, L. Nie, A. Bhirde, Z. Wang, Z. Liu, G. Niu, W. Wang, X. Chen, Theranostics 2014, 4, 229.

[208] L. Feng, S. Zhang, Z. Liu, Nanoscale 2011, 3, 1252.

[209] M. A. Lucherelli, Y. Yu, G. Reina, G. Abellán, E. Miyako, A. Bianco, Angew. Chem. $2020,132,14138$.

[210] G. Reina, D. Iglesias, P. Samorì, A. Bianco, Adv. Mater. 2021, 2007847.

[211] a) Z. Qi, P. Bharate, C.-H. Lai, B. Ziem, C. Böttcher, A. Schulz, F. Beckert, B. Hatting, R. Mülhaupt, P. H. Seeberger, R. Haag, Nano Lett. 2015, 15, 6051; b) G. Reina, S. Peng, L. Jacquemin, A. F. Andrade, A. Bianco, ACS Nano 2020, 14, 9364.

[212] Y. Liang, W.-H. Lai, Z. Miao, S.-L. Chou, Small 2018, 14, 1702514.

[213] D. Wang, D. Choi, J. Li, Z. Yang, Z. Nie, R. Kou, D. Hu, C. Wang, L. V. Saraf, J. Zhang, I. A. Aksay, J. Liu, ACS Nano 2009, 3, 907.

[214] H. Wang, L.-F. Cui, Y. Yang, H. Sanchez Casalongue, J. T. Robinson, Y. Liang, Y. Cui, H. Dai, J. Am. Chem. Soc. 2010, 132, 13978.

[215] J. K. Lee, K. B. Smith, C. M. Hayner, H. H. Kung, Chem. Commun. (Cambridge, U. K.) 2010, 46, 2025.

[216] J. Luo, X. Zhao, J. Wu, H. D. Jang, H. H. Kung, J. Huang, J. Phys. Chem. Lett. 2012, 3, 1824.

[217] D. Y. W. Yu, P. V. Prikhodchenko, C. W. Mason, S. K. Batabyal, J. Gun, S. Sladkevich, A. G. Medvedev, O. Lev, Nat. Comm. 2013, 4, 2922.

[218] M. Liu, P. Zhang, Z. Qu, Y. Yan, C. Lai, T. Liu, S. Zhang, Nat. Comm. 2019, 10, 3917.

[219] X. Huang, X. Qi, F. Boey, H. Zhang, Chem. Soc. Rev. 2012, 41, 666.

[220] J. Yan, Z. Fan, T. Wei, W. Qian, M. Zhang, F. Wei, Carbon 2010, 48, 3825.

[221] L. Peng, X. Peng, B. Liu, C. Wu, Y. Xie, G. Yu, Nano Lett. 2013, 13, 2151.

[222] B. Xie, Y. Chen, M. Yu, T. Sun, L. Lu, T. Xie, Y. Zhang, Y. Wu, Carbon 2016, 99, 35.

[223] Q. Wu, Y. Xu, Z. Yao, A. Liu, G. Shi, ACS Nano 2010, 4, 1963.

[224] J. An, J. Liu, Y. Zhou, H. Zhao, Y. Ma, M. Li, M. Yu, S. Li, J. Phys. Chem. C 2012, 116, 19699.

[225] Y. Meng, K. Wang, Y. Zhang, Z. Wei, Adv. Mater. 2013, 25, 6985.

[226] S. Witomska, Z. Liu, W. Czepa, A. Aliprandi, D. Pakulski, P. Pawluć, A. Ciesielski, P. Samorì, J. Am. Chem. Soc. 2018, 141, 482. 


\section{WILEY-VCH}

[227] Z. Liu, H. Zhang, M. Eredia, H. Qiu, W. Baaziz, O. Ersen, A. Ciesielski, M. Bonn, H. I. Wang, P. Samorì, ACS Nano 2019, 13, 9431.

[228] M. Morales-Masis, S. De Wolf, R. Woods-Robinson, J. W. Ager, C. Ballif, Adv. Electron. Mater. 2017, 3, 1600529.

[229] M. Pandey, Z. Wang, G. Kapil, A. K. Baranwal, D. Hirotani, K. Hamada, S. Hayase, Adv. Eng. Mater. 2019, 21, 1900288.

[230] N. N. Rosli, M. A. Ibrahim, N. Ahmad Ludin, M. A. Mat Teridi, K. Sopian, Renewable Sustainable Energy Rev. 2019, 99, 83.

[231] a) K. Rana, J. Singh, J.-H. Ahn, J. Mater. Chem. C 2014, 2, 2646; b) Y. Ma, L. Zhi, Small Methods 2019, 3, 1800199.

[232] H. Bi, F. Huang, J. Liang, X. Xie, M. Jiang, Adv. Mater. 2011, 23, 3202.

[233] S. Bae, H. Kim, Y. Lee, X. Xu, J.-S. Park, Y. Zheng, J. Balakrishnan, T. Lei, H. Ri Kim, Y. I. Song, Y.-J. Kim, K. S. Kim, B. Özyilmaz, J.-H. Ahn, B. H. Hong, S. Iijima, Nat. Nanotechnol. 2010, 5, 574.

[234] X. Lin, J. Jia, N. Yousefi, X. Shen, J.-K. Kim, J. Mater. Chem. C 2013, 1, 6869.

[235] X. Miao, S. Tongay, M. K. Petterson, K. Berke, A. G. Rinzler, B. R. Appleton, A. F. Hebard, Nano Lett. 2012, 12, 2745.

[236] C.-L. Hsu, C.-T. Lin, J.-H. Huang, C.-W. Chu, K.-H. Wei, L.-J. Li, ACS Nano 2012, 6, 5031.

[237] a) Z. Liu, J. Li, Z.-H. Sun, G. Tai, S.-P. Lau, F. Yan, ACS Nano 2011, 6, 810; b) B. H. Lee, J.-H. Lee, Y. H. Kahng, N. Kim, Y. J. Kim, J. Lee, T. Lee, K. Lee, Adv. Funct. Mater. 2014, 24, 1847.

[238] J. Meyer, P. R. Kidambi, B. C. Bayer, C. Weijtens, A. Kuhn, A. Centeno, A. Pesquera, A. Zurutuza, J. Robertson, S. Hofmann, Sci. Rep. 2014, 4, 5380.

[239] H. Chang, G. Wang, A. Yang, X. Tao, X. Liu, Y. Shen, Z. Zheng, Adv. Funct. Mater. 2010, 20, 2893.

[240] G. Zhu, L. Pan, T. Lu, T. Xu, Z. Sun, J. Mater. Chem. 2011, 21, 14869.

[241] I. N. Kholmanov, C. W. Magnuson, R. Piner, J. Y. Kim, A. E. Aliev, C. Tan, T. Y. Kim, A. A. Zakhidov, G. Sberveglieri, R. H. Baughman, R. S. Ruoff, Adv. Mater. 2015, 27, 3053 .

[242] L. Dou, F. Cui, Y. Yu, G. Khanarian, S. W. Eaton, Q. Yang, J. Resasco, C. Schildknecht, K. Schierle-Arndt, P. Yang, ACS Nano 2016, 10, 2600. 


\section{WILEY-VCH}

[243] F. Xia, T. Mueller, Y.-m. Lin, A. Valdes-Garcia, P. Avouris, Nat. Nanotechnol. 2009, 4,839 .

[244] F. H. L. Koppens, T. Mueller, P. Avouris, A. C. Ferrari, M. S. Vitiello, M. Polini, Nat. Nanotechnol. 2014, 9, 780.

[245] Z. Ni, L. Ma, S. Du, Y. Xu, M. Yuan, H. Fang, Z. Wang, M. Xu, D. Li, J. Yang, W. Hu, X. Pi, D. Yang, ACS Nano 2017, 11, 9854.

[246] S.-Y. Chen, Y.-Y. Lu, F.-Y. Shih, P.-H. Ho, Y.-F. Chen, C.-W. Chen, Y.-T. Chen, W.H. Wang, Carbon 2013, 63, 23.

[247] K. K. Manga, J. Wang, M. Lin, J. Zhang, M. Nesladek, V. Nalla, W. Ji, K. P. Loh, Adv. Mater. 2012, 24, 1697.

[248] J. Du, H.-M. Cheng, Macromol. Chem. Phys. 2012, 213, 1060.

[249] a) G. Shi, S. Araby, C. T. Gibson, Q. Meng, S. Zhu, J. Ma, Adv. Funct. Mater. 2018, 28, 1706705; b) V. Palermo, I. A. Kinloch, S. Ligi, N. M. Pugno, Adv. Mater. 2016, 28, 6232 .

[250] I. A. Kinloch, J. Suhr, J. Lou, R. J. Young, P. M. Ajayan, Science 2018, 362, 547.

[251] A. Zurutuza, C. Marinelli, Nat. Nanotechnol. 2014, 9, 730.

[252] B. E. Deal, J. M. Early, J. Electrochem. Soc. 1979, 126, $20 \mathrm{C}$. 